\title{
Convenient Synthesis of Branched-Chain Glycosamines by Radical Addition of Nitromethane to Glycals
}

Elangovan Elamparuthi and Torsten Linker

\section{Supporting Information}

\section{Addition of nitromethane (2) to the glycals $3(\operatorname{method} C)$ :}

A solution of the benzyl protected glycal $3(1 \mathrm{mmol})$, potassium hydroxide $(112 \mathrm{mg}, 2 \mathrm{mmol}$, 2 equiv) and nitromethane (2) $(610 \mathrm{mg}, 10 \mathrm{mmol}, 10$ equiv) in methanol (5 mL) was cooled to $0{ }^{\circ} \mathrm{C}$ under an argon atmosphere. At this temperature a solution of cerium(IV) ammonium nitrate (4 equiv) in methanol $(10 \mathrm{~mL})$ was added drop-wise over a period of $2 \mathrm{~h}$ until TLC showed complete conversion of the starting material. After stirring for $30 \mathrm{~min}$ at $0{ }^{\circ} \mathrm{C}$, an icecold diluted solution of sodium bisulfate $(50 \mathrm{~mL})$ and ammonium chloride $(20 \mathrm{~mL})$ was added, and the mixture was extracted with dichloromethane $(3 \times 100 \mathrm{~mL})$. The combined organic extracts were dried over sodium sulfate $\left(\mathrm{Na}_{2} \mathrm{SO}_{4}\right)$ and concentrated. The crude product was purified by column chromatography (cyclohexane /ethyl acetate $6: 1$ ) to afford the 2-deoxy-2- $C$-nitromethyl-pyranosides $\mathbf{4}$ in analytically pure form.

\section{Methyl 3,4,6-tri- $O$-benzyl-2-deoxy-2- $C$-nitromethyl- $\beta$-D-glucopyranoside (equat-4b):}

$\mathrm{R}_{\mathrm{f}}=0.32$ (cyclohexane / ethyl acetate $\left.2: 1\right) ;[\alpha]_{\mathrm{D}}{ }^{20}=+10.4\left(\mathrm{c}=1.02\right.$ in $\left.\mathrm{CHCl}_{3}\right) ;{ }^{1} \mathrm{H}-\mathrm{NMR}$ $\left(500 \mathrm{MHz}, \mathrm{CDCl}_{3}\right): \delta=2.28(\mathrm{dddd}, \mathrm{J}=11.0,8.8,4.4,4.1 \mathrm{~Hz}, 1 \mathrm{H}, 2-\mathrm{H}), 3.46(\mathrm{ddd}, \mathrm{J}=9.6$, 6.3, 3.4 Hz, $1 \mathrm{H}, 5-\mathrm{H}), 3.51$ (s, $3 \mathrm{H}, \mathrm{OMe}), 3.65$ (dd, J = 11.0, 8.7 Hz, $1 \mathrm{H}, 3-\mathrm{H}), 3.71$ (dd, J = 9.6, 8.7 Hz, $1 \mathrm{H}, 4-\mathrm{H}), 3.75$ (dd, J = 12.4, 3.4 Hz, $1 \mathrm{H}, 6-\mathrm{H}), 3.77$ (dd, J = 12.4, 6.3 Hz, $1 \mathrm{H}$, 6'-H), 4.40 (d, J = 8.8 Hz, $1 \mathrm{H}, 1-\mathrm{H}), 4.56$ (d, J = 12.0 Hz, $\left.1 \mathrm{H}, \mathrm{CH}_{2}-\mathrm{Ph}\right), 4.57$ (dd, J = 12.6, $4.1 \mathrm{~Hz}, 1 \mathrm{H}, 7-\mathrm{H}), 4.59\left(\mathrm{~d}, \mathrm{~J}=12.0 \mathrm{~Hz}, 1 \mathrm{H}, \mathrm{CH}_{2}-\mathrm{Ph}\right), 4.61\left(\mathrm{~d}, \mathrm{~J}=11.0 \mathrm{~Hz}, 1 \mathrm{H}, \mathrm{CH}_{2}-\mathrm{Ph}\right)$, $4.62\left(\mathrm{~d}, \mathrm{~J}=11.0 \mathrm{~Hz}, 1 \mathrm{H}, \mathrm{CH}_{2}-\mathrm{Ph}\right), 4.65$ (dd, J = 12.6, $4.4 \mathrm{~Hz}, 1 \mathrm{H}, 7$ '-H), 4.79 (d, J = 11.0 $\left.\mathrm{Hz}, 1 \mathrm{H}, \mathrm{CH}_{2}-\mathrm{Ph}\right), 4.93\left(\mathrm{~d}, \mathrm{~J}=11.0 \mathrm{~Hz}, 1 \mathrm{H}, \mathrm{CH}_{2}-\mathrm{Ph}\right), 7.18-7.36$ (m, $15 \mathrm{H}$, arom. $\left.\mathrm{H}\right) ;{ }^{13} \mathrm{C}-$ NMR (75 MHz, $\left.\mathrm{CDCl}_{3}\right): \delta=46.6(\mathrm{~d}, \mathrm{C}-2), 57.1$ (q, OMe), $68.6(\mathrm{t}, \mathrm{C}-6), 72.1\left(\mathrm{t}, \mathrm{CH}_{2}-\mathrm{NO}_{2}\right)$, 73.5, 74.7 (2t, $\mathrm{CH}_{2}-\mathrm{Ph}$ ), 75.1 (d, C-3), 75.1 (t, $\mathrm{CH}_{2}-\mathrm{Ph}$ ), 75.6, 79.6, 80.4, 101.9 (3d, C-4, C-5, C-1), 127.6, 127.7, 127.8, 127.9, 127.9, 128.0, 128.4, 128.5, 128.6 (m, arom. C-H), 137.7, 137.8, 138.0 (3s, arom. C-CH $2 \mathrm{O}$ ); IR (film): $v=3030,2914,1555,1496,1453,1380,1209$, $1093,911,738,698,617,463 \mathrm{~cm}^{-1}$; elemental analysis cald (\%) for $\mathrm{C}_{29} \mathrm{H}_{33} \mathrm{NO}_{7}(507)$ : $\mathrm{C}$ : 68.62, H: 6.55, N: 2.76; found: C: 68.49, H: 6.67, N: 2.80 . 
Methyl 3,4,6-tri-O-benzyl-2-deoxy-2- $C$-nitromethyl- $\alpha$-D-mannopyranoside ( $a x-4 b)$ :

$\mathrm{R}_{\mathrm{f}}=0.35$ (cyclohexane / ethyl acetate $\left.2: 1\right) ;[\alpha]_{\mathrm{D}}{ }^{20}=+68.0\left(\mathrm{c}=1.00\right.$ in $\left.\mathrm{CHCl}_{3}\right) ;{ }^{1} \mathrm{H}-\mathrm{NMR}$ $\left(500 \mathrm{MHz}, \mathrm{CDCl}_{3}\right): \delta=3.01(\mathrm{dddd}, \mathrm{J}=6.9,5.8,3.4,1.3 \mathrm{~Hz}, 1 \mathrm{H}, 2-\mathrm{H}), 3.27(\mathrm{~s}, 3 \mathrm{H}, \mathrm{OMe})$, $3.41(\mathrm{dd}, \mathrm{J}=9.3,8.4 \mathrm{~Hz}, 1 \mathrm{H}, 4-\mathrm{H}), 3.57(\mathrm{dd}, \mathrm{J}=11.8,3.4 \mathrm{~Hz}, 1 \mathrm{H}, 6-\mathrm{H}), 3.64$ (ddd, J = 8.4, 5.1. $3.4 \mathrm{~Hz}, 1 \mathrm{H}, 5-\mathrm{H}), 3.67$ (dd, J = 11.8, $5.1 \mathrm{~Hz}, 1 \mathrm{H}, 6$ '-H), 4.08 ( dd, J = 9.3, 5.8 Hz, $1 \mathrm{H}$, 3-H), 4.36 (dd, J = 13.4, 6.9 Hz, 1 H, 7-H), 4.39 (d, J = 1.3 Hz, 1 H, 1-H), 4.41 (d, J = 12.0 $\left.\mathrm{Hz}, 1 \mathrm{H}, \mathrm{CH}_{2}-\mathrm{Ph}\right), 4.48\left(\mathrm{~d}, \mathrm{~J}=11.2 \mathrm{~Hz}, 1 \mathrm{H}, \mathrm{CH}_{2}-\mathrm{Ph}\right), 4.52$ (d, J = 11.2 Hz, $\left.1 \mathrm{H}, \mathrm{CH}_{2}-\mathrm{Ph}\right)$, $4.55\left(\mathrm{~d}, \mathrm{~J}=12.0 \mathrm{~Hz}, 1 \mathrm{H}, \mathrm{CH}_{2}-\mathrm{Ph}\right), 4.59$ (d, J = 11.2 Hz, $\left.1 \mathrm{H}, \mathrm{CH}_{2}-\mathrm{Ph}\right), 4.68$ (dd, J = 13.4, 3.4 $\left.\mathrm{Hz}, 1 \mathrm{H}, 7^{\prime}-\mathrm{H}\right), 4.71$ (d, J = $\left.11.2 \mathrm{~Hz}, 1 \mathrm{H}, \mathrm{CH}_{2}-\mathrm{Ph}\right), 7.16-7.4$ (m, $15 \mathrm{H}$, arom. H); ${ }^{13} \mathrm{C}-\mathrm{NMR}$ $\left(75 \mathrm{MHz}, \mathrm{CDCl}_{3}\right): \delta=44.6$ (d, C-2), 55.1 (q, OMe), 68.4 (t, C-6), 70.9 (d, C-3), 73.5 (t, $\mathrm{CH}_{2^{-}}$ $\mathrm{NO}_{2}$ ), 73.6, 74.8, 75.1 (3t, $\mathrm{CH}_{2}-\mathrm{Ph}$ ), 77.8, 79.5, 98.0 (3d, C-4, C-5, C-1), 127.7, 127.7, 127.8, 127.8, 127.9, 128.0, 128.1, 128.4, 128.6 (m, arom. C-H), 137.7, 137.9, 137.9 (3s, arom. C$\mathrm{CH}_{2} \mathrm{O}$ ); IR (Film) : $v=3030,2911,1551,1496,1453,1377,1206,1097,1063,988,911,734$, $695,675,621,572 \mathrm{~cm}^{-1}$; elemental analysis cald (\%) for $\mathrm{C}_{29} \mathrm{H}_{33} \mathrm{NO}_{7}(507)$ : C 68.62, $\mathrm{H} \mathrm{6.55}$, N 2.76; found: C 68.51, H 6.56, N 2.48.

\section{Methyl 3,4,6-tri- $O$-benzyl-2-deoxy-2- $C$-nitromethyl- $\beta$-D-galactopyranoside (equat-4c):}

$\mathrm{R}_{\mathrm{f}}=0.54$ (cyclohexane / ethyl acetate 2:1); m.p : $127-128{ }^{0} \mathrm{C} ;[\alpha]_{\mathrm{D}}{ }^{20}=+8.4(\mathrm{c}=0.98$ in $\left.\mathrm{CHCl}_{3}\right) ;{ }^{1} \mathrm{H}-\mathrm{NMR}\left(500 \mathrm{MHz}, \mathrm{CDCl}_{3}\right): \delta=2.71$ (dddd, $\left.\mathrm{J}=9.8,8.8,4.1,3.6 \mathrm{~Hz}, 1 \mathrm{H}, 2-\mathrm{H}\right)$, 3.30 (dd, J = 13.0, 1.0 Hz, $1 \mathrm{H}, 6-\mathrm{H}), 3.50$ (s, $3 \mathrm{H}, \mathrm{OMe}$ ), 3.56 (dd, J = 7.4, $3.0 \mathrm{~Hz}, 1 \mathrm{H}, 4-\mathrm{H})$, $3.73(\mathrm{dd}, \mathrm{J}=7.4,2.0,1.0 \mathrm{~Hz}, 1 \mathrm{H}, 5-\mathrm{H}), 4.17(\mathrm{dd}, \mathrm{J}=13.0,2.0 \mathrm{~Hz}, 1 \mathrm{H}, 6$-H), 4.33 (dd, J = 9.8, 3.2 Hz, $1 \mathrm{H}, 3-\mathrm{H}), 4.34$ (d, J = 8.8 Hz, $1 \mathrm{H}, 1-\mathrm{H}), 4.52$ (d, J = 11.2 Hz, $\left.1 \mathrm{H}, \mathrm{CH}_{2}-\mathrm{Ph}\right), 4.62$ (d, J = 12.4 Hz, $\left.1 \mathrm{H}, \mathrm{CH}_{2}-\mathrm{Ph}\right), 4.69$ (dd, J = 12.6, 3.6 Hz, $\left.1 \mathrm{H}, 7-\mathrm{H}\right), 4.69$ (d, J = 12.4 Hz, $1 \mathrm{H}$, $\left.\mathrm{CH}_{2}-\mathrm{Ph}\right), 4.73\left(\mathrm{dd}, \mathrm{J}=12.6,4.4 \mathrm{~Hz}, 1 \mathrm{H}, 7^{\prime}-\mathrm{H}\right), 4.73$ (d, J = 12.4 Hz, $\left.1 \mathrm{H}, \mathrm{CH}_{2}-\mathrm{Ph}\right), 4.74$ (d, J $\left.=11.2 \mathrm{~Hz}, 1 \mathrm{H}, \mathrm{CH}_{2}-\mathrm{Ph}\right), 4.77\left(\mathrm{~d}, \mathrm{~J}=12.4 \mathrm{~Hz}, 1 \mathrm{H}, \mathrm{CH}_{2}-\mathrm{Ph}\right), 7.25-7.38(\mathrm{~m}, 15 \mathrm{H}$, arom. $\mathrm{H}) ;{ }^{13} \mathrm{C}-\mathrm{NMR}\left(75 \mathrm{MHz}, \mathrm{CDCl}_{3}\right): \delta=42.3$ (d, C-2), 57.4 (q, OMe), 69.1 (t, C-6), 71.0 (d, C-3), 72.2 (t, $\left.\mathrm{CH}_{2}-\mathrm{NO}_{2}\right), 72.6$ (t, $\left.\mathrm{CH}_{2}-\mathrm{Ph}\right), 73.9$, (d, C-4), 74.0, 74.9 (2t, $\left.\mathrm{CH}_{2}-\mathrm{Ph}\right), 78.3,102.0$ (2d, C-5, C-1), 128.0, 128.2, 128.3, 128.4, 128.5, 128.6, 128.9, 130.0 (m, arom. C-H), 138.0, 138.3, 138.6 (3s, arom. C-CH ${ }_{2} \mathrm{O}$ ); IR (Film) : $v=3031,2862,1549,1495,1451,1352,1205$, $1159,1095,1066,1025,1000,910,750,696,640,596 \mathrm{~cm}^{-1}$; elemental analysis cald (\%) for $\mathrm{C}_{29} \mathrm{H}_{33} \mathrm{NO}_{7}$ (507) : C 68.62, H 6.55, N 2.76; found: C 68.59, H 6.51, N 2.77. 


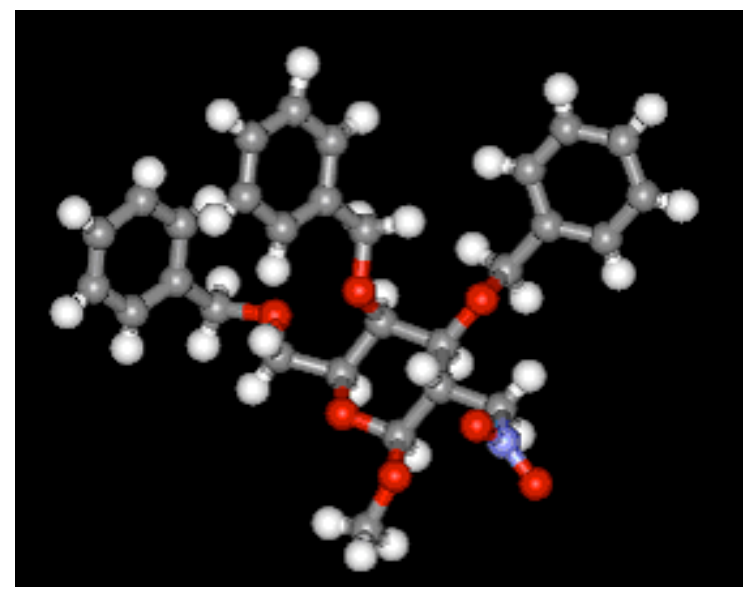

X-ray structure of the $\beta$-D-galactopyranoside

\section{Methyl 3,4,6-tri- $O$-benzyl-2-deoxy-2- $C$-nitromethyl- $\alpha$-D-talopyranoside (ax-4c):}

$\mathrm{R}_{\mathrm{f}}=0.62$ (cyclohexane / ethyl acetate 2:1); $[\alpha]_{\mathrm{D}}{ }^{20}=+26.5\left(\mathrm{c}=1.00\right.$ in $\left.\mathrm{CHCl}_{3}\right) ;{ }^{1} \mathrm{H}-\mathrm{NMR}$ $\left(500 \mathrm{MHz}, \mathrm{CDCl}_{3}\right): \delta=2.84(\mathrm{dddd}, \mathrm{J}=8.7,5.2,4.5,4.2, \mathrm{~Hz}, 1 \mathrm{H}, 2-\mathrm{H}), 3.37(\mathrm{~s}, 3 \mathrm{H}, \mathrm{OMe})$, $3.61(\mathrm{dd}, \mathrm{J}=9.3,6.4 \mathrm{~Hz}, 6-\mathrm{H}), 3.66\left(\mathrm{dd}, \mathrm{J}=9.3,6.5 \mathrm{~Hz}, 1 \mathrm{H}, 6{ }^{\prime}-\mathrm{H}\right), 3.85(\mathrm{dd}, \mathrm{J}=2.7,1.2 \mathrm{~Hz}$, $1 \mathrm{H}, 4-\mathrm{H}), 3.94(\mathrm{ddd}, \mathrm{J}=7.5,6.5,1.2 \mathrm{~Hz}, 1 \mathrm{H}, 5-\mathrm{H}), 4.06(\mathrm{dd}, \mathrm{J}=5.2,2.7 \mathrm{~Hz}, 1 \mathrm{H}, 3-\mathrm{H})$, $4.47\left(\mathrm{~d}, \mathrm{~J}=12.0 \mathrm{~Hz}, 1 \mathrm{H}, \mathrm{CH}_{2}-\mathrm{Ph}\right), 4.52\left(\mathrm{~d}, \mathrm{~J}=11.3 \mathrm{~Hz}, 1 \mathrm{H}, \mathrm{CH}_{2}-\mathrm{Ph}\right), 4.56$ (d, J = 12.0 Hz, 1 $\mathrm{H}, \mathrm{CH}_{2}-\mathrm{Ph}$ ), 4.61 (d, J = 12.0 Hz, $\left.1 \mathrm{H}, \mathrm{CH}_{2}-\mathrm{Ph}\right), 4.70$ (d, J = 12.0 Hz, $\left.1 \mathrm{H}, \mathrm{CH}_{2}-\mathrm{Ph}\right), 4.80-$ 4.83 (m, 2 H, 7-H, 7'-H), 4.84 (d, J = 8.7 Hz, 1 H, 1-H), 4.85 (d, J = 11.3 Hz, 1 H, CH2-Ph), $7.26-7.40\left(\mathrm{~m}, 15 \mathrm{H}\right.$, arom. H); ${ }^{13} \mathrm{C}-\mathrm{NMR}\left(75 \mathrm{MHz}, \mathrm{CDCl}_{3}\right): \delta=40.9(\mathrm{~d}, \mathrm{C}-2), 55.6(\mathrm{q}$, OMe), 69.4 (t, C-6), 69.7 (d, C-3), 71.2 (t, $\left.\mathrm{CH}_{2}-\mathrm{NO}_{2}\right)$, 73.8, 74.0 (2t, $\left.\mathrm{CH}_{2}-\mathrm{Ph}\right), 74.3,74.4$ (2d, C-4, C-5), 75.3 (t, $\left.\mathrm{CH}_{2}-\mathrm{Ph}\right), 100.1$ (d, C-1), 127.8, 128.1, 128.2, 128.2, 128.3, 128.8, 128.8, 130.0 (m, arom. C-H),138.1, 138.4, 138.7 (3s, arom. C-CH ${ }_{2} \mathrm{O}$ ); IR (Film) : v = 3030, 2910, 1547, 1496, 1453, 1357, 1205, 1096, 1051, 1026, 959, 806, 733, 695, $601596 \mathrm{~cm}^{-1}$; elemental analysis cald (\%) for $\mathrm{C}_{29} \mathrm{H}_{33} \mathrm{NO}_{7}(507)$ : C 68.62, H 6.55, N 2.76; found: C 68.59, H $6.82, \mathrm{~N} 2.63$.

\section{Methyl 3,4-di-O-benzyl-2-deoxy-2-C-nitromethyl- $\beta$-D-xylopyranoside (equat-4d):}

$\mathrm{R}_{\mathrm{f}}=0.41$ (cyclohexane / ethyl acetate $\left.2: 1\right) ;[\alpha]_{\mathrm{D}}{ }^{20}=+45.7\left(\mathrm{c}=0.98\right.$ in $\left.\mathrm{CHCl}_{3}\right) ;{ }^{1} \mathrm{H}-\mathrm{NMR}$ $\left(500 \mathrm{MHz}, \mathrm{CDCl}_{3}\right): \delta=2.24(\mathrm{dddd}, \mathrm{J}=9.5,8.1,4.7,4.5 \mathrm{~Hz}, 1 \mathrm{H}, 2-\mathrm{H}), 3.25(\mathrm{dd}, \mathrm{J}=11.8,5.8$ $\mathrm{Hz}, 1 \mathrm{H}, 5-\mathrm{H}), 3.46$ (s, 3H, OMe), 3.57 (dd, J = 9.5, 8.1 Hz $1 \mathrm{H}, 3-\mathrm{H}), 3.66$ (ddd, J = 9.5, 5.8, $4.9 \mathrm{~Hz}, 1 \mathrm{H}, 4-\mathrm{H}), 4.03$ (dd, J = 11.8, $\left.4.9 \mathrm{~Hz}, 1 \mathrm{H}, 5^{\prime}-\mathrm{H}\right), 4.36$ (d, J = 8.1 Hz, $\left.1 \mathrm{H}, 1-\mathrm{H}\right), 4.57-$ 4.60 (m, 2 H, 6-H, 6'-H), 4.63 (d, J = 11.5 Hz, 1 H, CH $-\mathrm{Ph}), 4.64$ (d, J = 11.5 Hz, 1 H, $\mathrm{CH}_{2}-$ $\mathrm{Ph}), 4.67\left(\mathrm{~d}, \mathrm{~J}=11.5 \mathrm{~Hz}, 1 \mathrm{H}, \mathrm{CH}_{2}-\mathrm{Ph}\right), 4.94\left(\mathrm{~d}, \mathrm{~J}=11.5 \mathrm{~Hz}, 1 \mathrm{H}, \mathrm{CH}_{2}-\mathrm{Ph}\right), 7.25-7.36$ (m, 
$10 \mathrm{H}$, arom. H); ${ }^{13} \mathrm{C}-\mathrm{NMR}\left(75 \mathrm{MHz}, \mathrm{CDCl}_{3}\right): \delta=45.9$ (d, C-2), 57.3 (q, OMe), 63.6 (t, C-5), 73.0 (t, $\mathrm{CH}_{2}-\mathrm{NO}_{2}$ ), 73.2, 75.1, (2t, $\mathrm{CH}_{2}-\mathrm{Ph}$ ), 77.9, 79.8, 102.2 (3d, C-3, C-4, C-1), 128.2, 128.4, 128.4, 128.5, 128.9, 130.0, (m, arom. C-H), 138.2, 138.3 (2s, arom. C-CH $\left.\mathrm{C}_{2} \mathrm{O}\right)$; IR (Film) $: v=3030,2875,1552,1453,1378,1206,1070,1027,997,901,734,696,620,587$ $\mathrm{cm}^{-1}$; elemental analysis cald (\%) for $\mathrm{C}_{21} \mathrm{H}_{25} \mathrm{NO}_{6}(387)$ : C 65.10, H 6.50, N 3.62; found: $\mathrm{C}$ 64.95, H 6.61, N 3.49.

\section{Methyl 3,4-di-O-benzyl-2-deoxy-2- $C$-nitromethyl- $\alpha$-D-lyxopyranoside ( $a x$-4d):}

$\mathrm{R}_{\mathrm{f}}=0.62$ (cyclohexane / ethyl acetate $\left.2: 1\right) ;[\alpha]_{\mathrm{D}}{ }^{20}=+52.7\left(\mathrm{c}=0.99\right.$ in $\left.\mathrm{CHCl}_{3}\right) ;{ }^{1} \mathrm{H}-\mathrm{NMR}$ $\left(500 \mathrm{MHz}, \mathrm{CDCl}_{3}\right): \delta=2.66$ (dddd, $\left.\mathrm{J}=8.5,4.8,4.7,3.6 \mathrm{~Hz}, 1 \mathrm{H}, 2-\mathrm{H}\right), 3.34(\mathrm{~s}, 3 \mathrm{H}, \mathrm{OMe})$, $3.56(\mathrm{dd}, \mathrm{J}=10.0,4.3 \mathrm{~Hz}, 1 \mathrm{H}, 5-\mathrm{H}), 3.66$ (ddd, J = 9.6, 4.3, $4.1 \mathrm{~Hz}, 1 \mathrm{H}, 4-\mathrm{H}), 3.77$ (dd, J = 10.1, 4.1 Hz, 1 H, 5'-H), 4.54 (dd, J = 13.4, 4.8 Hz, 1 H, 6-H), 4.57 (dd, J = 13.4, 3.6 Hz, 1 H, 6'-H), 4.62 (d, J = 11.2 Hz, $\left.1 \mathrm{H}, \mathrm{CH}_{2}-\mathrm{Ph}\right), 4.66$ (d, J = 11.3 Hz, $1 \mathrm{H}, \mathrm{CH}_{2}-\mathrm{Ph}$ ), 4.68 (d, J = 8.5 Hz, $1 \mathrm{H}, 1-\mathrm{H}), 4.69$ (d, J = 11.2 Hz, $\left.1 \mathrm{H}, \mathrm{CH}_{2}-\mathrm{Ph}\right), 4.70$ (dd, J = 9.6, $\left.4.7 \mathrm{~Hz}, 1 \mathrm{H}, 3-\mathrm{H}\right), 4.95$ $\left(\mathrm{d}, \mathrm{J}=11.3 \mathrm{~Hz}, 1 \mathrm{H}, \mathrm{CH}_{2}-\mathrm{Ph}\right), 7.27-7.38$ (m, $10 \mathrm{H}$, arom. $\left.\mathrm{H}\right) ;{ }^{13} \mathrm{C}-\mathrm{NMR}\left(75 \mathrm{MHz}, \mathrm{CDCl}_{3}\right): \delta$ $=44.1(\mathrm{~d}, \mathrm{C}-2), 55.2(\mathrm{q}, \mathrm{OMe}), 60.3(\mathrm{t}, \mathrm{C}-5), 72.8\left(\mathrm{t}, \mathrm{CH}_{2}-\mathrm{NO}_{2}\right), 73.5,74.9\left(2 \mathrm{t}, \mathrm{CH}_{2}-\mathrm{Ph}\right), 76.5$, 79.2, 98.2 (3d, C-3, C-4, C-1), 127.6, 128.0, 128.0, 128.5, (m, arom. C-H), 137.9, 138.0 (2s, arom. C-CH $\mathrm{CH}_{2} \mathrm{O}$ ); IR (Film) : $v=3030,2932,1550,1453,1377,1265,1204,1092,1045,1027$, $952,735,696 \mathrm{~cm}^{-1}$; elemental analysis cald (\%) for $\mathrm{C}_{21} \mathrm{H}_{25} \mathrm{NO}_{6}(387): \mathrm{C} 65.10, \mathrm{H} 6.50, \mathrm{~N}$ 3.62; found: C 64.97, H 6.42, N 3.29.

\section{Methyl 3,4-di-O-benzyl-2-deoxy-2- $C$-nitromethyl- $\alpha$-D-arbinopyranoside (ax-4e):}

$\mathrm{R}_{\mathrm{f}}=0.41$ (cyclohexane / ethyl acetate 2:1); m.p : 127-128 ${ }^{0} \mathrm{C} ;[\alpha]_{\mathrm{D}}{ }^{20}=-46.1(\mathrm{c}=1.01$ in $\left.\mathrm{CHCl}_{3}\right) ;{ }^{1} \mathrm{H}-\mathrm{NMR}\left(500 \mathrm{MHz}, \mathrm{CDCl}_{3}\right): \delta=2.72(\mathrm{dddd}, \mathrm{J}=9.1,8.4,6.5,4.0 \mathrm{~Hz}, 1 \mathrm{H}, 2-\mathrm{H})$, 3.31 (dd, J = 13.0, 2.0 Hz, 1 H, 5-H), 3.51 (s, 3 H, OMe), 3.58 (dd, J = 9.1, 2.7 Hz, 1 H, 3-H), 3.75 (ddd, J = 2.7, 2.2, $2.0 \mathrm{~Hz}, 1 \mathrm{H}, 4-\mathrm{H}), 4.19(\mathrm{dd}, \mathrm{J}=13.0,2.2 \mathrm{~Hz}, 1 \mathrm{H}, 5-\mathrm{H}), 4.35$ (d, J = $\left.11.2 \mathrm{~Hz}, 1 \mathrm{H}, \mathrm{CH}_{2}-\mathrm{Ph}\right), 4.36(\mathrm{~d}, \mathrm{~J}=8.4 \mathrm{~Hz}, 1 \mathrm{H}, 1-\mathrm{H}), 4.54$ (d, J = 11.2 Hz, $\left.1 \mathrm{H}, \mathrm{CH}_{2}-\mathrm{Ph}\right)$, $4.63\left(\mathrm{~d}, \mathrm{~J}=12.3 \mathrm{~Hz}, 1 \mathrm{H}, \mathrm{CH}_{2}-\mathrm{Ph}\right), 4.70(\mathrm{dd}, \mathrm{J}=12.5,4.1 \mathrm{~Hz}, 1 \mathrm{H}, 6-\mathrm{H}), 4.74$ (dd, J = 12.5, $4.6 \mathrm{~Hz}, 1 \mathrm{H}, 6$ ' $-\mathrm{H}), 4.75$ (d, J = $\left.12.3 \mathrm{~Hz}, 1 \mathrm{H}, \mathrm{CH}_{2}-\mathrm{Ph}\right), 7.27-7.40$ (m, $10 \mathrm{H}$, arom. H); ${ }^{13} \mathrm{C}-$ NMR (75 MHz, $\mathrm{CDCl}_{3}$ ) : $\delta=42.4$ (d, C-2), 57.4 (q, OMe), 63.8 (t, C-5), 69.9 (d, C-3), 71.5, 71.5, (2t, $\left.\mathrm{CH}_{2}-\mathrm{Ph}\right), 72.5$ (t, $\mathrm{CH}_{2}-\mathrm{NO}_{2}$ ), 76.4, 102.3 (2d, C-4, C-1), 128.2, 128.4, 128.4, 128.8, 128.9 (m, arom. C-H), 138.2, 138.4,(2s, arom. C-CH $\left.{ }_{2} \mathrm{O}\right)$; IR (Film) : v = 3029, 2867, 1535, $1453,1380,1350,1255,1225,1122,1093,1001,878,796,740,696,649,619,564,492,425$ 
$\mathrm{cm}^{-1}$; elemental analysis cald (\%) for $\mathrm{C}_{21} \mathrm{H}_{25} \mathrm{NO}_{6}$ (387) : C 65.10, H 6.50, N 3.62; found: C 65.12, H 6.58, N 3.66.

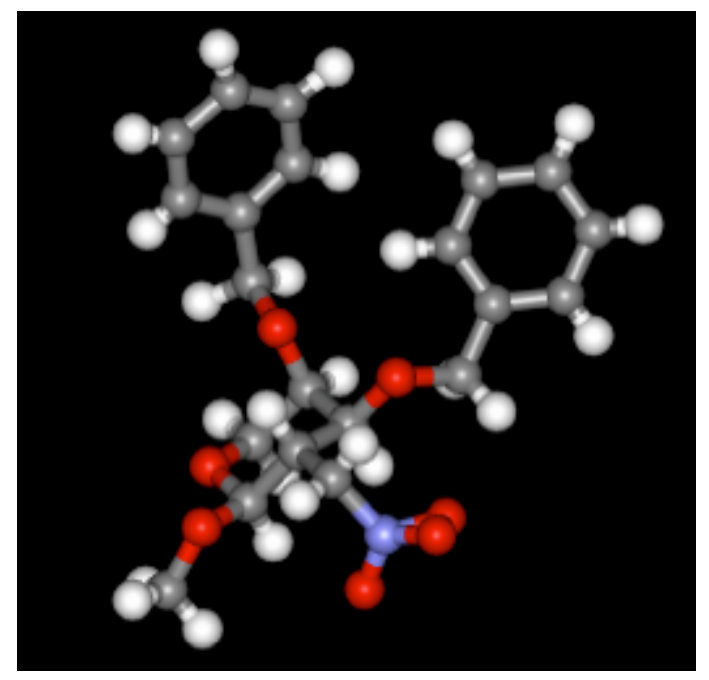

X-ray structure of the $\alpha$-D-arbinopyranoside

\section{Methyl 3,4-di- $O$-benzyl-2-deoxy-2- $C$-nitromethyl- $\beta$-D-ribopyranoside (equat-4e):}

$\mathrm{R}_{\mathrm{f}}=0.54$ (cyclohexane / ethyl acetate $\left.2: 1\right) ;[\alpha]_{\mathrm{D}}{ }^{20}=-60.7\left(\mathrm{c}=0.98\right.$ in $\left.\mathrm{CHCl}_{3}\right) ;{ }^{1} \mathrm{H}-\mathrm{NMR}$ $\left(500 \mathrm{MHz}, \mathrm{CDCl}_{3}\right): \delta=2.84(\mathrm{dddd}, \mathrm{J}=7.8,4.8,4.1,2.8 \mathrm{~Hz}, 1 \mathrm{H}, 2-\mathrm{H}), 3.38$ (s, 3H, OMe), $3.69(\mathrm{dd}, \mathrm{J}=12.1,2.2 \mathrm{~Hz}, 1 \mathrm{H}, 5-\mathrm{H}), 3.74$ (ddd, J = 2.5, 2.2, $1.0 \mathrm{~Hz}, 1 \mathrm{H}, 4-\mathrm{H}), 3.82$ (dd, J = 12.1, $\left.2.5 \mathrm{~Hz}, 1 \mathrm{H}, 5^{\prime}-\mathrm{H}\right), 4.03$ (dd, J = 7.8, $\left.1.0 \mathrm{~Hz}, 1 \mathrm{H}, 3-\mathrm{H}\right), 4.58$ (d, J = $12.0 \mathrm{~Hz}, 1 \mathrm{H}, \mathrm{CH}_{2}-$ Ph), $4.62-4.68$ (m, 2 H, 6-H, 6'-H), 4.69 (d, J = 12.0 Hz, 1 H, CH $-\mathrm{Ph}$ ), 4.73 (d, J = 12.0 Hz, $\left.1 \mathrm{H}, \mathrm{CH}_{2}-\mathrm{Ph}\right), 4.75\left(\mathrm{~d}, \mathrm{~J}=12.0 \mathrm{~Hz}, 1 \mathrm{H}, \mathrm{CH}_{2}-\mathrm{Ph}\right), 4.78$ (d, J = 4.8 Hz, $\left.1 \mathrm{H}, 1-\mathrm{H}\right), 7.27$ - 7.40 $\left(\mathrm{m}, 10 \mathrm{H}\right.$, arom. H); ${ }^{13} \mathrm{C}-\mathrm{NMR}\left(75 \mathrm{MHz}, \mathrm{CDCl}_{3}\right): \delta=41.7$ (d, C-2), 55.6 (q, OMe) 60.9 (t, C-5), 71.6 (t, $\mathrm{CH}_{2}-\mathrm{NO}_{2}$ ), 72.0, (t, $\mathrm{CH}_{2}-\mathrm{Ph}$ ), 72.5 (d, C-3), 73.3, (t, $\left.\mathrm{CH}_{2}-\mathrm{Ph}\right), 73.9,99.8$ (2d, C4, C-1), 127.5, 127.6, 127.7, 127.8, 128.5, 128.5 (m, arom. C-H), 137.9, 138.1,(2s, arom. C$\mathrm{CH}_{2} \mathrm{O}$ ); IR (Film) : $v=3030,2872,1546,1452,1379,1355,1268,1127,1053,939,905,733$, 696, 634, $595 \mathrm{~cm}^{-1}$; elemental analysis cald (\%) for $\mathrm{C}_{21} \mathrm{H}_{25} \mathrm{NO}_{6}(387): \mathrm{C} 65.10, \mathrm{H} 6.50, \mathrm{~N}$ 3.62; found: C 65.02, H 6.72, N 3.24.

\section{Methyl}

3,6,8,9,10,12-hexa- $O$-benzyl-2-deoxy-2- $C$-nitromethyl- $\beta$-D-maltopyranoside (equat-4f):

$\mathrm{R}_{\mathrm{f}}=0.50$ (cyclohexane / ethyl acetate 2:1); $[\alpha]_{\mathrm{D}}{ }^{20}=+41.2\left(\mathrm{c}=1.00\right.$ in $\left.\mathrm{CHCl}_{3}\right) ;{ }^{1} \mathrm{H}-\mathrm{NMR}$ $\left(500 \mathrm{MHz}, \mathrm{CDCl}_{3}\right): \delta=2.44(\mathrm{dddd}, \mathrm{J}=9.7,8.8,5.2,4.4 \mathrm{~Hz}, 1 \mathrm{H}, 2-\mathrm{H}), 3.48(\mathrm{~s}, 3 \mathrm{H}, \mathrm{OMe})$, $3.51(\mathrm{dd}, \mathrm{J}=10.7,2.4 \mathrm{~Hz}, 1 \mathrm{H}, 12-\mathrm{H}), 3.54$ (ddd, J = 9.6, 5.3, $4.1 \mathrm{~Hz}, 1 \mathrm{H}, 5-\mathrm{H}), 3.60$ (dd, J = 10.7, 3.6 Hz, 1 H, 12'-H), 3.64 (dd, J = 10.7, 9.6 Hz, 1 H, 4-H), 3.75 (dd, J = 10.7, 8.8 Hz, 1 H, 9-H), 3.77 (ddd, J = 10.2, 3.6, 2.4 Hz, 1 H, 11-H), 3.84 (dd, J = 10.2, 8.8 Hz, 1 H, 10-H), 
$3.92(\mathrm{dd}, \mathrm{J}=10.7,3.6 \mathrm{~Hz}, 1 \mathrm{H}, 8-\mathrm{H}), 3.96(\mathrm{dd}, \mathrm{J}=11.2,4.1 \mathrm{~Hz}, 1-\mathrm{H}, 6-\mathrm{H}), 4.10(\mathrm{~d}, \mathrm{~J}=8.2$ Hz, 1 H, 1-H), 4.36 - 4.38 (m, 2 H, 13-H, 13'-H), 4.45 (dd, J = 11.2, 5.3 Hz, 1 H, 6'-H), 4.47 $(\mathrm{dd}, \mathrm{J}=10.7,8.8 \mathrm{~Hz}, 1 \mathrm{H}, 3-\mathrm{H}), 4,52\left(\mathrm{~d}, \mathrm{~J}=12.0 \mathrm{~Hz}, 1 \mathrm{H}, \mathrm{CH}_{2}-\mathrm{Ph}\right), 4,52$ (d, J = 11.2, Hz, 1 $\left.\mathrm{H}, \mathrm{CH}_{2}-\mathrm{Ph}\right), 4,53$ (d, J = 12.0 Hz, $\left.1 \mathrm{H}, \mathrm{CH}_{2}-\mathrm{Ph}\right), 4.54$ (d, J = $\left.12.0 \mathrm{~Hz}, 1 \mathrm{H}, \mathrm{CH}_{2}-\mathrm{Ph}\right), 4.55$ (d, J $\left.=12.0 \mathrm{~Hz}, 1 \mathrm{H}, \mathrm{CH}_{2}-\mathrm{Ph}\right), 4.57\left(\mathrm{~d}, \mathrm{~J}=11.2 \mathrm{~Hz}, 1 \mathrm{H}, \mathrm{CH}_{2}-\mathrm{Ph}\right), 4.62\left(\mathrm{~d}, \mathrm{~J}=12.0 \mathrm{~Hz}, 1 \mathrm{H}, \mathrm{CH}_{2}-\right.$ $\mathrm{Ph}), 4.78$ (d, J = 11.2 Hz, $\left.1 \mathrm{H}, \mathrm{CH}_{2}-\mathrm{Ph}\right), 4.80$ (d, J = 11.2 Hz, $\left.1 \mathrm{H}, \mathrm{CH}_{2}-\mathrm{Ph}\right), 4.82$ (d, J = 11.2 $\left.\mathrm{Hz}, 1 \mathrm{H}, \mathrm{CH}_{2}-\mathrm{Ph}\right), 4.87$ (d, J = 11.2 Hz, $\left.1 \mathrm{H}, \mathrm{CH}_{2}-\mathrm{Ph}\right), 5.00$ (d, J = 11.2 Hz, $\left.1 \mathrm{H}, \mathrm{CH}_{2}-\mathrm{Ph}\right)$, $5.28(\mathrm{~d}, \mathrm{~J}=3.6 \mathrm{~Hz}, 1 \mathrm{H}, 7-\mathrm{H}), 7.13-7.30$ (m, $30 \mathrm{H}$, arom. H); ${ }^{13} \mathrm{C}-\mathrm{NMR}\left(75 \mathrm{MHz}, \mathrm{CDCl}_{3}\right): \delta$ $=45.3(\mathrm{~d}, \mathrm{C}-2), 56.9$ (q, OMe), 68.5, $69.2(2 \mathrm{t}, \mathrm{C}-6, \mathrm{C}-12), 71.2(\mathrm{~d}, \mathrm{C}-3), 72.4\left(\mathrm{t}, \mathrm{CH}_{2}-\mathrm{NO}_{2}\right)$, 72.5, 73.3, 73.4, 73.5, 75.0 (5t, $\left.\mathrm{CH}_{2}-\mathrm{Ph}\right), 75.5$ (d, C-4), 75.5 (t, $\left.\mathrm{CH}_{2}-\mathrm{Ph}\right), 75.6,77.8,78.7$, 79.7, 82.0, 97.4, 101.4 (7d, C-5, C- 8, C-9, C-10, C-11, C-7, C-1), 127.5, 127.6, 127.7, 127.8, 127.9, 128.0, 128.3, 128.3, 128.4, 128.4 (m, arom. C-H), 137.8, 137.8, 138.0, 138.3, 138.3, 138.6 (6s, arom. C-CH $\mathrm{CH}_{2} \mathrm{O}$ ); IR (Film) : $v=3031,2873,1540,1487,1402,1394,1209,1087$, $976,738,687,632,543,521 \mathrm{~cm}^{-1}$; elemental analysis cald (\%) for $\mathrm{C}_{56} \mathrm{H}_{61} \mathrm{NO}_{12}(939)$ : $\mathrm{C}$ : 71.55, H 6.54, N 1.49; found: C : 71.67, H 6.98, N 1.62.

\section{Methyl 3,6,8,9,10,12-hexa- $O$-benzyl-2-deoxy-2-epi-2- $C$-nitromethyl- $\alpha$-D-maltopyranoside} (ax-4f):

$\mathrm{R}_{\mathrm{f}}=0.55$ (cyclohexane / ethyl acetate $\left.2: 1\right) ;[\alpha]_{\mathrm{D}}{ }^{20}=+61.6\left(\mathrm{c}=0.99\right.$ in $\left.\mathrm{CHCl}_{3}\right) ;{ }^{1} \mathrm{H}-\mathrm{NMR}$ $\left(500 \mathrm{MHz}, \mathrm{CDCl}_{3}\right): \delta=2.80(\mathrm{dddd}, \mathrm{J}=9.4,8.2,4.4,3.4 \mathrm{~Hz}, 1 \mathrm{H}, 2-\mathrm{H}), 3.31(\mathrm{~s}, 3 \mathrm{H}, \mathrm{OMe})$, 3.50 (ddd, J = 9.8, 6.4, 3.6 Hz, 1 H, 5-H), 3.58 (dd, J = 10.8, 4.3 Hz, 1 H, 12-H), 3.63 (dd, J = 9.4, $9.8 \mathrm{~Hz}, 1 \mathrm{H}, 4-\mathrm{H}), 3.66$ (dd, J = 10.8, 4.1 Hz, $1 \mathrm{H}, 12^{\prime}-\mathrm{H}$ ), 3.74 (ddd, J = 10.4, 4.3, $4.1 \mathrm{~Hz}$, $1 \mathrm{H}, 11-\mathrm{H}), 3.77$ (dd, J = 10.1, 8.6 Hz, $1 \mathrm{H}, 9-\mathrm{H}), 3.93$ (dd, J = 10.4, 8.6 Hz, $1 \mathrm{H}, 10-\mathrm{H}), 4.03$ (dd, J = 10.1, 3.5 Hz, 1 H, 8-H), 4. 11 (dd, J = 9.4, 3.4 Hz, $1 \mathrm{H}, 3-\mathrm{H}), 4.31$ (dd, J = 13.4, 3.8 Hz, 1 H, 13-H), 4.34 (dd, J = 13.4, 3.8 Hz, 1 H, 13'-H), 4.38 - 4.39 (m, 2 H, 6-H, 6'-H), 4.41 $(\mathrm{d}, \mathrm{J}=8.2 \mathrm{~Hz}, 1 \mathrm{H}, 1-\mathrm{H}), 4,46\left(\mathrm{~d}, \mathrm{~J}=11.5 \mathrm{~Hz}, 1 \mathrm{H}, \mathrm{CH}_{2}-\mathrm{Ph}\right), 4,47$ (d, J = $11.5 \mathrm{~Hz}, 1 \mathrm{H}, \mathrm{CH}_{2}-$ $\mathrm{Ph}$ ), 4,50 (d, J = 12.3 Hz, $\left.1 \mathrm{H}, \mathrm{CH}_{2}-\mathrm{Ph}\right), 4.52$ (d, J = 11.5 Hz, $\left.1 \mathrm{H}, \mathrm{CH}_{2}-\mathrm{Ph}\right), 4.53$ (d, J = 11.5 $\mathrm{Hz}, 1 \mathrm{H}, \mathrm{CH}_{2}-\mathrm{Ph}$ ), 4.56 (d, J = 12.3 Hz, $\left.1 \mathrm{H}, \mathrm{CH}_{2}-\mathrm{Ph}\right), 4.59$ (d, J = 12.3 Hz, $1 \mathrm{H}, \mathrm{CH}_{2}-\mathrm{Ph}$ ), $4.76\left(\mathrm{~d}, \mathrm{~J}=11.5 \mathrm{~Hz}, 1 \mathrm{H}, \mathrm{CH}_{2}-\mathrm{Ph}\right), 4.77$ (d, J = 12.3 Hz, $\left.1 \mathrm{H}, \mathrm{CH}_{2}-\mathrm{Ph}\right), 4.82$ (d, J = 11.5 Hz, 1 $\mathrm{H}, \mathrm{CH}_{2}-\mathrm{Ph}$ ), 4.87 (d, J = 11.5 Hz, $\left.1 \mathrm{H}, \mathrm{CH}_{2}-\mathrm{Ph}\right), 5.00$ (d, J = 11.5 Hz, $\left.1 \mathrm{H}, \mathrm{CH}_{2}-\mathrm{Ph}\right), 5.30$ (d, $\mathrm{J}=3.5 \mathrm{~Hz}, 1 \mathrm{H}, 7-\mathrm{H}), 7.14-7.32\left(\mathrm{~m}, 30 \mathrm{H}\right.$, arom. H); ${ }^{13} \mathrm{C}-\mathrm{NMR}\left(75 \mathrm{MHz}, \mathrm{CDCl}_{3}\right): \delta=43.7$ (d, C-2), 55.2 (q, OMe), 68.7, 69.0 (2t, C-6, C-12), 70.9, 71.2 (2d, C-3, C-4), 73.1 (t, $\mathrm{CH}_{2-}$ $\mathrm{NO}_{2}$ ), 73.2, 73.3, 73.3, 73.4, 75.0, 75.5 (6t, $\left.\mathrm{CH}_{2}-\mathrm{Ph}\right), 76.1,77.7,77.8,79.9,81.9,97.5,97.7$ (7d, C-5, C-8, C-9, C-10, C-11, C-1, C-7), 127.4, 127.5, 127.6, 127.6, 127.7, 127.8, 127.8, 
128.0, 128.3, 128.3, 128.5 (m, arom. C-H), 137.9, 138.0, 138.0, 138.3, 138.3, 138.6 (6s, arom. C-CH $\mathrm{CH}_{2} \mathrm{O}$ ); IR (Film) : $v=3030,2862,1571,1462,1441,1372,1237,1075,976,731,696$, $654,543 \mathrm{~cm}^{-1}$; elemental analysis cald (\%) for $\mathrm{C}_{56} \mathrm{H}_{61} \mathrm{NO}_{12}(939)$ : C 71.55, H 6.54, N 1.49; found: C 71.43, H 6.86, N 1.65.

\section{Methyl $\quad 3,6,8,9,10,12-h e x a-O$-benzyl-2-deoxy-2- $C$-nitromethyl- $\beta$-D-lactopyranoside (equat-4g):}

$\mathrm{R}_{\mathrm{f}}=0.51$ (cyclohexane / ethyl acetate $\left.2: 1\right) ;[\alpha]_{\mathrm{D}}{ }^{20}=+8.1\left(\mathrm{c}=1.01\right.$ in $\left.\mathrm{CHCl}_{3}\right) ;{ }^{1} \mathrm{H}-\mathrm{NMR}(500$ $\left.\mathrm{MHz}, \mathrm{CDCl}_{3}\right): \delta=2.13(\mathrm{dddd}, \mathrm{J}=9.4,8.4,4.4,4.1 \mathrm{~Hz}, 1 \mathrm{H}, 2-\mathrm{H}), 3.27(\mathrm{dd}, \mathrm{J}=11.3,3.4 \mathrm{~Hz}$, $1 \mathrm{H}, 12-\mathrm{H}), 3.29$ (dd, J = 6.8, $2.4 \mathrm{~Hz}, 1 \mathrm{H}, 4-\mathrm{H}), 3.32$ (ddd, J = 6.8, 4.4, $1.4 \mathrm{~Hz}, 1 \mathrm{H}, 5-\mathrm{H}$ ), 3.42 (s, 3 H, OMe), 3.40 - 3.52 (m, 1 H, 11-H), 3.58 (dd, J = 11.3, 8.5 Hz, 1 H, 12'-H), 3.61 $(\mathrm{dd}, \mathrm{J}=11.0,1.4 \mathrm{~Hz}, 1-\mathrm{H}, 6-\mathrm{H}), 3.70(\mathrm{dd}, \mathrm{J}=9.7,2.2 \mathrm{~Hz}, 1 \mathrm{H}, 10-\mathrm{H}), 3.80(\mathrm{dd}, \mathrm{J}=11.0,4.4$ Hz, 1-H, 6'-H), 3.83 (dd, J = 2.4, 2.2 Hz, 1 H, 9-H), 3.96 (dd, J = 9.0, 2.4 Hz, 1 H, H-8), 4, 17 $\left(\mathrm{d}, \mathrm{J}=11.5 \mathrm{~Hz}, 1 \mathrm{H}, \mathrm{CH}_{2}-\mathrm{Ph}\right), 4.28\left(\mathrm{~d}, \mathrm{~J}=11.5 \mathrm{~Hz}, 1 \mathrm{H}, \mathrm{CH}_{2}-\mathrm{Ph}\right), 4.32$ (dd, J = 9.4, $2.4 \mathrm{~Hz}, 1$ H, H-3), 4.33 - 4.38 (m, 2 H, 13-H, 13'-H), 4.36 (d, J = 11.5 Hz, 1 H, $\mathrm{CH}_{2}-\mathrm{Ph}$ ), 4.43 (d, J = 8.4 Hz, $1 \mathrm{H}, 1-\mathrm{H}), 4.46$ (d, J = 11.5 Hz, $\left.1 \mathrm{H}, \mathrm{CH}_{2}-\mathrm{Ph}\right), 4.52$ (d, J = 12.1 Hz, $\left.1 \mathrm{H}, \mathrm{CH}_{2}-\mathrm{Ph}\right), 4.55$ $\left(\mathrm{d}, \mathrm{J}=12.1 \mathrm{~Hz}, 1 \mathrm{H}, \mathrm{CH}_{2}-\mathrm{Ph}\right), 4.59\left(\mathrm{~d}, \mathrm{~J}=12.1 \mathrm{~Hz}, 1 \mathrm{H}, \mathrm{CH}_{2}-\mathrm{Ph}\right), 4.66$ (d, J = 12.1 Hz, $1 \mathrm{H}$, $\left.\mathrm{CH}_{2}-\mathrm{Ph}\right), 4.71\left(\mathrm{~d}, \mathrm{~J}=11.5 \mathrm{~Hz}, 1 \mathrm{H}, \mathrm{CH}_{2}-\mathrm{Ph}\right), 4.75\left(\mathrm{~d}, \mathrm{~J}=12.1 \mathrm{~Hz}, 1 \mathrm{H}, \mathrm{CH}_{2}-\mathrm{Ph}\right), 4.87(\mathrm{~d}, \mathrm{~J}=$ $11.5 \mathrm{~Hz}, 1 \mathrm{H}, \mathrm{CH}_{2}-\mathrm{Ph}$ ), 4.89 (d, J = 11.5 Hz, $1 \mathrm{H}, \mathrm{CH}_{2}-\mathrm{Ph}$ ), 5.12 (d, J = $\left.10.2 \mathrm{~Hz}, 1 \mathrm{H}, 7-\mathrm{H}\right)$, $7.05-7.25$ (m, $30 \mathrm{H}$, arom. H); ${ }^{13} \mathrm{C}-\mathrm{NMR}\left(75 \mathrm{MHz}, \mathrm{CDCl}_{3}\right): \delta=46.5$ (d, C-2), 57.0 (q, OMe), 67.9, 68.1 (2t, C-6, C-12), 72.3 (t, $\mathrm{CH}_{2}-\mathrm{NO}_{2}$ ), 72.7, 73.1 (2t, $\left.\mathrm{CH}_{2}-\mathrm{Ph}\right), 73.1$ (d, C-3 ), 73.4 (t, $\mathrm{CH}_{2}-\mathrm{Ph}$ ), 73.7 (d, C- 4) 74.7, 74.9 (2t, $\mathrm{CH}_{2}-\mathrm{Ph}$ ), 75.3 (d, C- 5), 75.3 (t, $\mathrm{CH}_{2}-\mathrm{Ph}$ ), 77.2, 77.4, 80.0, 82.4, 101.4, 102.6 (6d, C-8, C-9, C- 10, C- 11, C-1, C-7), 127.3, 127.4, 127.5, $127.5,127.6,127.7,127.8,127.9,128.0,128.1,128.2,128.3,128.4$ (m, arom. C-H), 138.1, 138.2, 138.4, 138.5, 138.7, 139.0 (6s, arom. C-CH ${ }_{2} \mathrm{O}$ ); IR (Film) : v = 3028, 2862, 1555, $1495,1452,1361,1207,1046,909,731,694,600,568,555 \mathrm{~cm}^{-1}$; elemental analysis cald (\%) for $\mathrm{C}_{56} \mathrm{H}_{61} \mathrm{NO}_{12}$ (939) : C 71.55, H 6.54, N 1.49; found: C 71.32, H 6.81, N 1.60.

\section{Methyl 3,6,8,9,10,12-hexa- $O$-benzyl-2-deoxy2-epi-2-C-nitromethyl- $\alpha$-D-lactopyranoside $(a x-4 g):$}

$\mathrm{R}_{\mathrm{f}}=0.52$ (cyclohexane / ethyl acetate $\left.2: 1\right) ;[\alpha]_{\mathrm{D}}{ }^{20}=+19.6\left(\mathrm{c}=0.99\right.$ in $\left.\mathrm{CHCl}_{3}\right) ;{ }^{1} \mathrm{H}-\mathrm{NMR}$ $\left(500 \mathrm{MHz}, \mathrm{CDCl}_{3}\right): \delta=3.03(\mathrm{dddd}, \mathrm{J}=9.1,8.1,5.9,3.5 \mathrm{~Hz}, 1 \mathrm{H}, 2-\mathrm{H}), 3.37$ (s, $\left.3 \mathrm{H}, \mathrm{OMe}\right)$, 3.37 (ddd, J = 4.6, 3.8, 2.8 Hz, $1 \mathrm{H}, 5-\mathrm{H}), 3.42$ (dd, J = 4.6, $3.1 \mathrm{~Hz}, 1 \mathrm{H}, 4-\mathrm{H}), 3.48$ (dd, J = 10.8, $5.4 \mathrm{~Hz}, 1 \mathrm{H}, 12-\mathrm{H}), 3.52$ (ddd, J = 9.2, 5.4, $2.0 \mathrm{~Hz}, 1 \mathrm{H}, 11-\mathrm{H}), 3.58$ (dd, J = 10.8, 2.0 
Hz, $\left.1 \mathrm{H}, 12^{\prime}-\mathrm{H}\right), 3.68$ (dd, J = 9.2, 4.0 Hz, $\left.1 \mathrm{H}, 10-\mathrm{H}\right), 3.76$ (dd, J = 10.7, 8.7 Hz, $1 \mathrm{H}, \mathrm{H}-8$ ), $3.82(\mathrm{dd}, \mathrm{J}=11.0,3.8 \mathrm{~Hz}, 1-\mathrm{H}, 6-\mathrm{H}), 3.90$ (dd, J = 11.0, $2.8 \mathrm{~Hz}, 1 \mathrm{H}, 6$ '-H), 4.02 (dd, J = 9.1, $3.1 \mathrm{~Hz}, 1 \mathrm{H}, \mathrm{H}-3), 4.26$ (d, J = 11.8, Hz, $1 \mathrm{H}, \mathrm{CH}_{2}-\mathrm{Ph}$ ), 4.30 (d, J = 11.5, Hz, $1 \mathrm{H}, \mathrm{CH}_{2}-\mathrm{Ph}$ ), $4.32(\mathrm{~d}, \mathrm{~J}=8.1 \mathrm{~Hz}, 1 \mathrm{H}, 1-\mathrm{H}), 4.33\left(\mathrm{~d}, \mathrm{~J}=11.8, \mathrm{~Hz}, 1 \mathrm{H}, \mathrm{CH}_{2}-\mathrm{Ph}\right), 4.35(\mathrm{dd}, \mathrm{J}=8.7,4.0 \mathrm{~Hz}, 1$ $\mathrm{H}, 9-\mathrm{H}), 4.40\left(\mathrm{~d}, \mathrm{~J}=12.1 \mathrm{~Hz}, 1 \mathrm{H}, \mathrm{CH}_{2}-\mathrm{Ph}\right), 4.54$ (d, J = 11.5 Hz, $\left.1 \mathrm{H}, \mathrm{CH}_{2}-\mathrm{Ph}\right), 4.57$ (d, J = $\left.12.1 \mathrm{~Hz}, 1 \mathrm{H}, \mathrm{CH}_{2}-\mathrm{Ph}\right), 4.58\left(\mathrm{~d}, \mathrm{~J}=11.5 \mathrm{~Hz}, 1 \mathrm{H}, \mathrm{CH}_{2}-\mathrm{Ph}\right), 4.62$ (d, J = $11.5 \mathrm{~Hz}, 1 \mathrm{H}, \mathrm{CH}_{2}-$

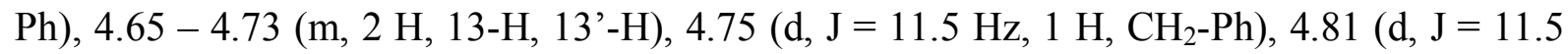
$\left.\mathrm{Hz}, 1 \mathrm{H}, \mathrm{CH}_{2}-\mathrm{Ph}\right), 4.85\left(\mathrm{~d}, \mathrm{~J}=11.5 \mathrm{~Hz}, 1 \mathrm{H}, \mathrm{CH}_{2}-\mathrm{Ph}\right), 4.97$ (d, J = $\left.11.5 \mathrm{~Hz}, 1 \mathrm{H}, \mathrm{CH}_{2}-\mathrm{Ph}\right)$, $5.16(\mathrm{~d}, \mathrm{~J}=10.7 \mathrm{~Hz}, 1 \mathrm{H}, 7-\mathrm{H}), 7.21-7.36$ (m, $30 \mathrm{H}$, arom. H); ${ }^{13} \mathrm{C}-\mathrm{NMR}\left(75 \mathrm{MHz}, \mathrm{CDCl}_{3}\right)$ : $\delta=42.4(\mathrm{~d}, \mathrm{C}-2), 57.2(\mathrm{q}, \mathrm{OMe}), 68.4$ (t, C-6), 68.7 (t, C-12), 71.0 (d, C-3 ), 72.7 (t, $\mathrm{CH}_{2-}$ $\mathrm{NO}_{2}$ ), 72.7, 72.9, 73.3, 73.5, (4t, $\left.\mathrm{CH}_{2}-\mathrm{Ph}\right), 73.5$ (d, C-4 ), 73.8 (d, C-5 ), 74.5 (d, C-8 ), 74.6, 75.2 (2t, $\left.\mathrm{CH}_{2}-\mathrm{Ph}\right), 75.4$ (d, C-9 ), 80.0 (d, C-10 ), 82.4 (d, C-11 ), 98.9 (d, C-1), 103.3 (d, C7), 127.4, 127.6, 127.7, 127.8, 128.1, 128.2, 128.3 (m, arom. C-H), 138.1, 138.2, 138.4, 138.5, 138.8, 138.8 (6s, arom. C- $\mathrm{CH}_{2} \mathrm{O}$ ); IR (Film) : $v=3033,2799,1547,1487,1465,1321$, $1256,1135,967,721,687,643,554 \mathrm{~cm}^{-1}$; elemental analysis cald (\%) for $\mathrm{C}_{56} \mathrm{H}_{61} \mathrm{NO}_{12}(939)$ : C 71.55, H 6.54, N 1.49; found: C 71.93, H 6.59, N 1.74.

\section{Reduction of the nitro group with lithium aluminium hydride $\left(\mathrm{LiAlH}_{4}\right)(\operatorname{method} \mathrm{D})$ :}

The 2-deoxy-2- $C$-nitromethyl-pyranoside 4 (1 mmol) was dissolved in tetrahydrofuran (THF) $(5 \mathrm{~mL})$ and lithium aluminum hydride $(76 \mathrm{mg}, 2 \mathrm{mmol})$ was added to the solution under an argon atmosphere. The mixture was heated under reflux for $1 \mathrm{~h}$ until TLC showed complete conversion. The reaction mixture was quenched with water and extracted by ethyl acetate. The combined organic extracts were dried over anhydrous sodium sulfate. After evaporation of the solvents the crude product was acetylated with acetic anhydride $(1 \mathrm{~mL})$ and pyridine $(4$ $\mathrm{mL}$ ) for $1.5 \mathrm{~h}$ to afford after concentration and column chromatography (ethyl acetate /methanol 9:1) the protected amides 7 in analytically pure form.

\section{Methyl 2-acetamidomethyl-3,4,6-tri- $O$-benzyl-2-deoxy- $\beta$-D-glucopyranoside (7b):}

$\mathrm{R}_{\mathrm{f}}=0.65$ (ethyl acetate /methanol 9:1); m.p : $113-114{ }^{0} \mathrm{C} ;[\alpha]_{\mathrm{D}}{ }^{20}=+32.5\left(\mathrm{c}=1.00\right.$ in $\left.\mathrm{CHCl}_{3}\right)$; ${ }^{1} \mathrm{H}-\mathrm{NMR}\left(500 \mathrm{MHz}, \mathrm{CDCl}_{3}\right.$ ): $\delta=1.78$ (s, $\left.3 \mathrm{H}, \mathrm{Ac}\right), 1.82$ (dddd, $\mathrm{J}=10.4,8.7,4.6,4.1 \mathrm{~Hz}, 1 \mathrm{H}$, 2-H), 3.19 (ddd, J = 10.2, 4.7, 4.3 Hz, $1 \mathrm{H}, 5-\mathrm{H}), 3.40$ (dd, J = 10.4, 9.5 Hz, $1 \mathrm{H}, 3-\mathrm{H}), 3.45$ (dd, J = 12.6, 4.3 Hz, 1 H, 6-H), 3.54 (s, 3 H, OMe), 3.65 (dd, J = 12.6, 4.7 Hz, 1 H, 6-H'), 
3.71 (dd, J = 10.2, 9.5 Hz, 1 H, 4-H), 3.76 - 3.77 (m, 2 H, 7-H, 7'-H), 4.15 (d, J = 8.7 Hz. 1 H, 1-H), 4.58 (d, J = 12.2 Hz, $\left.1 \mathrm{H}, \mathrm{CH}_{2} \mathrm{Ph}\right), 4.63$ (d, J = 11.1 Hz, $\left.1 \mathrm{H}, \mathrm{CH}_{2} \mathrm{Ph}\right), 4.67$ (d, J = $\left.12.2 \mathrm{~Hz}, 1 \mathrm{H}, \mathrm{CH}_{2} \mathrm{Ph}\right), 4.70\left(\mathrm{~d}, \mathrm{~J}=11.1 \mathrm{~Hz}, 1 \mathrm{H}, \mathrm{CH}_{2} \mathrm{Ph}\right), 4.82\left(\mathrm{~d}, \mathrm{~J}=11.1 \mathrm{~Hz}, 1 \mathrm{H}, \mathrm{CH}_{2} \mathrm{Ph}\right)$, $4.94\left(\mathrm{~d}, \mathrm{~J}=11.1 \mathrm{~Hz}, 1 \mathrm{H}, \mathrm{CH}_{2} \mathrm{Ph}\right), 5.76(\mathrm{t}, \mathrm{J}=4.4 \mathrm{~Hz}, 1 \mathrm{H}, \mathrm{NH}), 7.21$ - 7.39 (m, $15 \mathrm{H}$, arom $\mathrm{H}) ;{ }^{13} \mathrm{C}-\mathrm{NMR}\left(75 \mathrm{MHz}, \mathrm{CDCl}_{3}\right): \delta=23.1(\mathrm{q}, \mathrm{Ac}), 37.8\left(\mathrm{t}, \mathrm{CH}_{2}-\mathrm{NH}\right), 47.3(\mathrm{~d}, \mathrm{C}-2), 56.8(\mathrm{q}$, OMe), 68.8 (t, C-6), 73.5, 74.6, 74.7 (3t, $\left.\mathrm{CH}_{2}-\mathrm{Ph}\right), 75.1,79.9,81.1,103.6$ (4d, C-3, C-4, C-5, C-1), 127.6, 127.7, 127.8, 128.0, 128.3,128.3,128.6 (m, arom. C-H), 137.9, 138.0, 138.1 (3q, arom. C-CH $\mathrm{CH}_{2} \mathrm{O}$ ), 169.8 (s, Ac); IR (Film) : $v=3292,2924,1650,1557,1356,1298,1104$, 1024, 964, 800, $730 \mathrm{~cm}^{-1}$; elemental analysis cald (\%) for $\mathrm{C}_{31} \mathrm{H}_{37} \mathrm{NO}_{6}(519)$ : C 71.65, $\mathrm{H} 7.18$, N 2.70; found: C 71.51, H 7.24, N 2.67.

\section{Methyl 2-acetamidomethyl-3,4,6-tri- $O$-benzyl-2-deoxy- $\beta$-D-galactopyranoside (7c):}

$\mathrm{R}_{\mathrm{f}}=0.67$ (ethyl acetate /methanol 9:1); m.p : 107-108 ${ }^{0} \mathrm{C} ;[\alpha]_{\mathrm{D}}{ }^{20}=+28.6\left(\mathrm{c}=1.00\right.$ in $\mathrm{CHCl}_{3}$ ); ${ }^{1} \mathrm{H}-\mathrm{NMR}\left(500 \mathrm{MHz}, \mathrm{CDCl}_{3}\right): \delta=1.78$ (s, $3 \mathrm{H}, \mathrm{Ac}$ ), 2.21 (dddd, J = 9.8, 8.7, 5.6, 4.8 Hz, $1 \mathrm{H}$, 2-H), $3.23(\mathrm{dd}, \mathrm{J}=11.0,5.6 \mathrm{~Hz}, 1 \mathrm{H}, 7-\mathrm{H}), 3.31$ (dd, J = 11.0, $4.8 \mathrm{~Hz}, 1 \mathrm{H}, 7$ '-H), 3.50 (s, OMe), $3.52(\mathrm{ddd}, \mathrm{J}=7.0,5.8,2.0 \mathrm{~Hz}, 1 \mathrm{H}, 5-\mathrm{H}), 3.58$ (dd, J = 11.4, $5.7 \mathrm{~Hz}, 1 \mathrm{H}, 6-\mathrm{H}), 3.65$ (dd, J = 11.4, 5.8 Hz, 1 H, 6'-H), 3.96 (dd, J = 3.6, 2.0 Hz, 1 H, 4-H), 4.10 (d, J = 8.7 Hz. 1 H, 1-H), 4.12 (dd, J = 9.8, 3.6 Hz, $1 \mathrm{H}, 3-\mathrm{H}), 4.41$ (d, J = 11.4 Hz, $\left.1 \mathrm{H}, \mathrm{CH}_{2} \mathrm{Ph}\right), 4.47$ (d, J = 11.8 $\left.\mathrm{Hz}, 1 \mathrm{H}, \mathrm{CH}_{2} \mathrm{Ph}\right), 4.51$ (d, J = 11.8 Hz, $1 \mathrm{H}, \mathrm{CH}_{2} \mathrm{Ph}$ ), 4.63 (d, J = 11.8 Hz, $1 \mathrm{H}, \mathrm{CH}_{2} \mathrm{Ph}$ ), 4.72 $\left(\mathrm{d}, \mathrm{J}=11.4 \mathrm{~Hz}, 1 \mathrm{H}, \mathrm{CH}_{2} \mathrm{Ph}\right), 4.89\left(\mathrm{~d}, \mathrm{~J}=11.8 \mathrm{~Hz}, 1 \mathrm{H}, \mathrm{CH}_{2} \mathrm{Ph}\right), 5.90(\mathrm{t}, \mathrm{J}=4.4 \mathrm{~Hz}, 1 \mathrm{H}$, $\mathrm{NH}), 7.26-7.40\left(\mathrm{~m}, 15 \mathrm{H}\right.$, arom H); ${ }^{13} \mathrm{C}-\mathrm{NMR}\left(75 \mathrm{MHz}, \mathrm{CDCl}_{3}\right): \delta=23.11$ (q, Ac), 38.0 (t, $\mathrm{CH}_{2}-\mathrm{NH}$ ), 42.2 (d, C-2), 56.7 (q, OMe), 68.9 (t, C-6), 70.2 (d, C-3), 71.2 (t, $\left.\mathrm{CH}_{2}-\mathrm{Ph}\right), 73.4$ (d, C-4), 73.5, 74.3 (2t, $\mathrm{CH}_{2}-\mathrm{Ph}$ ), 80.0, 103.8 (2d, C-5, C-1), 127.5, 127.7, 127.8, 128.0, 128.1, 128.4, 128.7 (m, arom. C-H), 137.4, 137.9, 138.5 (3q, arom, C- $\mathrm{CH}_{2} \mathrm{O}$ ), 169.8 (s, Ac); IR (Film) $: v=3062,2863,1721,1651,1520,1452,1365,1270,1066,1026,910,734,696,594$, $576 \mathrm{~cm}^{-1}$; elemental analysis cald (\%) for $\mathrm{C}_{31} \mathrm{H}_{37} \mathrm{NO}_{6}(519)$ : C 71.65, H 7.18, N 2.70; found: C 71.87, H 7.43, N 2.62.

\section{Methyl 2-acetamidomethyl-3,4-di- $O$-benzyl-2-deoxy- $\beta$-D-xylopyranoside (7d):}

$\mathrm{R}_{\mathrm{f}}=0.71$ (ethyl acetate /methanol 9:1); $[\alpha]_{\mathrm{D}}{ }^{20}=+7.5\left(\mathrm{c}=1.00\right.$ in $\left.\mathrm{CHCl}_{3}\right) ;{ }^{1} \mathrm{H}-\mathrm{NMR}(500$ $\mathrm{MHz}, \mathrm{CDCl}_{3}$ ): $\delta=1.72(\mathrm{dddd}, \mathrm{J}=10.5,8.1,5.5,4.7 \mathrm{~Hz}, 1 \mathrm{H}, 2-\mathrm{H}), 1.74$ (s, $\left.3 \mathrm{H}, \mathrm{Ac}\right), 3.24$ (ddd, J = 8.8, 5.6, 4.0 Hz, $1 \mathrm{H}, 4-\mathrm{H}), 3.38$ (dd, J = 11.0, 4.7 Hz, $1 \mathrm{H}, 6-\mathrm{H}), 3.48$ (s, OMe), 3.56 (dd, J = 11.0, $5.5 \mathrm{~Hz}, 1 \mathrm{H}, 6$ '-H), 3.62 (dd, J = 12.0, 4.0 Hz, $1 \mathrm{H}, 5-\mathrm{H}), 3.68$ (dd, J = 10.5, 8.8 $\mathrm{Hz}, 1 \mathrm{H}, 3-\mathrm{H}), 4.02\left(\mathrm{dd}, \mathrm{J}=12.0,5.6 \mathrm{~Hz}, 1 \mathrm{H}, 5^{\prime}-\mathrm{H}\right), 4.12$ (d, J = 8.1 Hz, $\left.1 \mathrm{H}, 1-\mathrm{H}\right), 4.65$ (d, J 
$\left.=11.8 \mathrm{~Hz}, 1 \mathrm{H}, \mathrm{CH}_{2} \mathrm{Ph}\right), 4.69\left(\mathrm{~d}, \mathrm{~J}=11.8 \mathrm{~Hz}, 1 \mathrm{H}, \mathrm{CH}_{2} \mathrm{Ph}\right), 4.71(\mathrm{~d}, \mathrm{~J}=11.8 \mathrm{~Hz}, 1 \mathrm{H}$, $\left.\mathrm{CH}_{2} \mathrm{Ph}\right), 4.94\left(\mathrm{~d}, \mathrm{~J}=11.8 \mathrm{~Hz}, 1 \mathrm{H}, \mathrm{CH}_{2} \mathrm{Ph}\right), 5.68(\mathrm{t}, \mathrm{J}=4.5 \mathrm{~Hz}, 1 \mathrm{H}, \mathrm{NH}), 7.27-7.39(\mathrm{~m}, 10 \mathrm{H}$, $\operatorname{arom~} \mathrm{H}) ;{ }^{13} \mathrm{C}-\mathrm{NMR}\left(75 \mathrm{MHz}, \mathrm{CDCl}_{3}\right): \delta=23.1$ (q, Ac), $38.2\left(\mathrm{t}, \mathrm{CH}_{2}-\mathrm{NH}\right), 46.1(\mathrm{~d}, \mathrm{C}-2), 56.6$ (q, OMe), 63.0 (t, C-5), 72.5, 74.2 (2t, $\left.\mathrm{CH}_{2} \mathrm{Ph}\right), 79.2,79.5,103.6$ (3d, C-3, C-4, C-1), 127.9, 127.9, 128.1, 128.5, 128.5, 128.6 (m, arom C-H), 137.9138 .1 (2q, arom $\left.\mathrm{C}-\mathrm{CH}_{2} \mathrm{O}\right), 169.9$ (s, Ac); IR (Film) $: v=3305,2926,1722,1650,1549,1452,13720,1262,1070,1027,736,697$, $596 \mathrm{~cm}^{-1}$; elemental analysis cald (\%) for $\mathrm{C}_{23} \mathrm{H}_{29} \mathrm{NO}_{5}$ (399) : C 69.15, H 7.32, N 3.51; found: C 69.24, H 7.53, N 3.24.

\section{Methyl 2-acetamidomethyl-3,4-di- $O$-benzyl-2-deoxy- $\alpha$-D-arabinopyranoside (7e):}

$\mathrm{R}_{\mathrm{f}}=0.50$ (ethyl acetate /methanol 9:1); $[\alpha]_{\mathrm{D}}{ }^{20}=-71.0\left(\mathrm{c}=1.00\right.$ in $\left.\mathrm{CHCl}_{3}\right) ;{ }^{1} \mathrm{H}-\mathrm{NMR}(500$ $\mathrm{MHz}, \mathrm{CDCl}_{3}$ ): $\delta=1.78$ (s, $3 \mathrm{H}, \mathrm{Ac}$ ), 2.23 (dddd, $\left.\mathrm{J}=10.5,8.4,5.6,4.8 \mathrm{~Hz}, 1 \mathrm{H}, 2-\mathrm{H}\right), 3.27$ $(\mathrm{dd}, \mathrm{J}=10.5,1.0 \mathrm{~Hz}, 1 \mathrm{H}, 3-\mathrm{H}), 3.32(\mathrm{dd}, \mathrm{J}=10.7,3.1 \mathrm{~Hz}, 1 \mathrm{H}, 5-\mathrm{H}), 3.37$ (dd, J = 11.1, 4.8 Hz, $1 \mathrm{H}, 6-\mathrm{H}), 3.51$ (s, 3H, OMe), 3.56 (dd, J = 11.1, 5.6 Hz, $1 \mathrm{H}, 6$ '-H), 3.72 (dd, J = 3.1, 2.4, $1.0 \mathrm{~Hz}, 1 \mathrm{H}, 4-\mathrm{H}), 4.04$ (d, J = 8.4 Hz, 1 H, 1-H), 4.19 (dd, J = 10.7, 2.4 Hz, 1 H, 5'-H), 4.29 $\left(\mathrm{d}, \mathrm{J}=11.5 \mathrm{~Hz}, 1 \mathrm{H}, \mathrm{CH}_{2} \mathrm{Ph}\right), 4.59\left(\mathrm{~d}, \mathrm{~J}=11.5 \mathrm{~Hz}, 1 \mathrm{H}, \mathrm{CH}_{2} \mathrm{Ph}\right), 4.64(\mathrm{~d}, \mathrm{~J}=12.5 \mathrm{~Hz}, 1 \mathrm{H}$, $\left.\mathrm{CH}_{2} \mathrm{Ph}\right), 4.81\left(\mathrm{~d}, \mathrm{~J}=12.5 \mathrm{~Hz}, 1 \mathrm{H}, \mathrm{CH}_{2} \mathrm{Ph}\right), 6.0(\mathrm{t}, \mathrm{J}=4.4 \mathrm{~Hz}, 1 \mathrm{H}, \mathrm{NH}), 7.27-7.40(\mathrm{~m}, 10 \mathrm{H}$, $\operatorname{arom~} \mathrm{H}) ;{ }^{13} \mathrm{C}-\mathrm{NMR}\left(75 \mathrm{MHz}, \mathrm{CDCl}_{3}\right): \delta=23.1$ (q, Ac), $38.5\left(\mathrm{t}, \mathrm{CH}_{2}-\mathrm{NH}\right), 42.3(\mathrm{~d}, \mathrm{C}-2), 56.7$ (q, OMe), 63.1 (t, C-5), 69.1 (d, C-3), 70.5, 70.9 (2t, $\left.\mathrm{CH}_{2} \mathrm{Ph}\right), 78.3,104.0$ (2d, C-4, C-1), $127.9,127.7,128.0,128.1,128.2,128.4,128.6(\mathrm{~m}$, arom C-H), 137.4, 138.1 (2q, arom C$\mathrm{CH}_{2} \mathrm{O}$ ), 170.0 (s, Ac); IR (Film) : $v=3325(\mathrm{NH}), 2921,2876,1642,1544,1450,1364,1085$, $1031,731 \mathrm{~cm}^{-1}$; elemental analysis cald (\%) for $\mathrm{C}_{23} \mathrm{H}_{29} \mathrm{NO}_{5}(399)$ : C 69.15, H 7.32, N 3.51; found: C 68.96, H 7.18, N 3.39.

\section{Methyl 2-acetamidomethyl-3,6,8,9,10,12-hexa- $O$-benzyl-2-deoxy- $\beta$-D-maltopyranoside} (7f):

$\mathrm{R}_{\mathrm{f}}=0.80$ (ethyl acetate /methanol 9:1); $[\alpha]_{\mathrm{D}}{ }^{20}=+47.8\left(\mathrm{c}=1.00\right.$ in $\left.\mathrm{CHCl}_{3}\right) ;{ }^{1} \mathrm{H}-\mathrm{NMR}(500$ $\mathrm{MHz}, \mathrm{CDCl}_{3}$ ): $\delta=1.73$ (s, $3 \mathrm{H}, \mathrm{Ac}$ ), 2.00 (dddd, $\left.\mathrm{J}=10.1,8.1,6.6,4.2 \mathrm{~Hz}, 1 \mathrm{H}, 2-\mathrm{H}\right), 3.16$ (dd, J = 11.2, 6.1 Hz, $1 \mathrm{H}, 12-\mathrm{H}), 3.40$ (ddd, J = 7.7, 4.0, $2.4 \mathrm{~Hz}, 1 \mathrm{H}, 5-\mathrm{H}), 3.60$ (dd, J = 11.2, $\left.3.7 \mathrm{~Hz}, 1 \mathrm{H}, 12^{\prime}-\mathrm{H}\right), 3.50$ (s, $\left.3 \mathrm{H}, \mathrm{OMe}\right), 3.52$ (dd, J = 10.2, $\left.6.6 \mathrm{~Hz}, 1 \mathrm{H}, 13-\mathrm{H}\right), 3.57$ (dd, J = 9.3, 7.7 Hz, 1 H, 4-H), 3.63 (ddd, J=8.2, 6.1, 3.7 Hz, 1 H, 11-H), 3.77 (dd, J = 10.2, 4.2 Hz, 1 H, 13'-H), 3.83 - 3.86 (dd, J = 10.6, 8.2 Hz, 1 H, 10-H), 3.88 (dd, J = 11.4, 4.0 Hz, 1 H, 6-H), 3.90 (dd, J = 11.4, 2.4 Hz, 1 H, 6'-H), 3.92 (dd, J = 8.2, 4.0 Hz, 1 H, H-8), 4.07 (dd, J = 10.6, $8.2 \mathrm{~Hz}, 1 \mathrm{H}, 9-\mathrm{H}), 4.12(\mathrm{dd}, \mathrm{J}=10.1,9.3 \mathrm{~Hz}, 1 \mathrm{H}, 3-\mathrm{H}), 4.18(\mathrm{~d}, \mathrm{~J}=8.1 \mathrm{~Hz}, 1 \mathrm{H}, 1-\mathrm{H}), 4.28$ 
$\left(\mathrm{d}, \mathrm{J}=12.0 \mathrm{~Hz}, 1 \mathrm{H}, \mathrm{CH}_{2}-\mathrm{Ph}\right), 4.38\left(\mathrm{~d}, \mathrm{~J}=12.0 \mathrm{~Hz}, 1 \mathrm{H}, \mathrm{CH}_{2}-\mathrm{Ph}\right), 4.47(\mathrm{~d}, \mathrm{~J}=11.1 \mathrm{~Hz}, 1 \mathrm{H}$, $\left.\mathrm{CH}_{2}-\mathrm{Ph}\right), 4.52\left(\mathrm{~d}, \mathrm{~J}=12.0 \mathrm{~Hz}, 1 \mathrm{H}, \mathrm{CH}_{2}-\mathrm{Ph}\right), 4.55$ (d, J = $\left.12.0 \mathrm{~Hz}, 1 \mathrm{H}, \mathrm{CH}_{2}-\mathrm{Ph}\right), 4.59$ (d, J = $\left.12.0 \mathrm{~Hz}, 1 \mathrm{H}, \mathrm{CH}_{2}-\mathrm{Ph}\right), 4.78\left(\mathrm{~d}, \mathrm{~J}=11.1 \mathrm{~Hz}, 1 \mathrm{H}, \mathrm{CH}_{2}-\mathrm{Ph}\right), 4.81$ (d, J = $11.1 \mathrm{~Hz}, 1 \mathrm{H}, \mathrm{CH}_{2}-$ $\mathrm{Ph}), 4.83\left(\mathrm{~d}, \mathrm{~J}=11.1 \mathrm{~Hz}, 1 \mathrm{H}, \mathrm{CH}_{2}-\mathrm{Ph}\right), 4.87$ (d, J = 11.1 Hz, $\left.1 \mathrm{H}, \mathrm{CH}_{2}-\mathrm{Ph}\right), 4.89$ (d, J = 11.1 $\mathrm{Hz}, 1 \mathrm{H}, \mathrm{CH}_{2}-\mathrm{Ph}$ ), 4.9 (d, J = 12.0 Hz, $\left.1 \mathrm{H}, \mathrm{CH}_{2}-\mathrm{Ph}\right), 5.33$ (d, J = 3.5 Hz, $\left.1 \mathrm{H}, 7-\mathrm{H}\right), 5.95$ (t, J $=4.5 \mathrm{~Hz}, 1 \mathrm{H}, \mathrm{NH}), 7.13-7.33\left(\mathrm{~m}, 30 \mathrm{H}\right.$, arom. H); ${ }^{13} \mathrm{C}-\mathrm{NMR}\left(75 \mathrm{MHz}, \mathrm{CDCl}_{3}\right): \delta=23.0$ (q, Ac), 38.2 (t, $\left.\mathrm{CH}_{2}-\mathrm{NH}\right), 445.0$ (d, C-2), 56.5 (q, OMe), 68.5, 69.6, (2t, C-6, C-12) 71.1 (d, C-3), 73.3 , $73.2,73.4$ (t, $\mathrm{CH}_{2}-\mathrm{Ph}$ ), 74.7 (d, C-4), 75.0 (t, $\left.\mathrm{CH}_{2}-\mathrm{Ph}\right), 75.3$ (d, C-5), 75.4 (t, $\mathrm{CH}_{2}-$ $\mathrm{Ph}), 77.7,79.7,80.0,81.8,97.1,103.1$ (6d, C-8, C-9, C-10, C-11, C-7, C-1), 127.5, 127.6, 127.6, 127.7, 127.8, 127.8, 128.0, 128.0, 128.2, 128.3, 128.3, 128.5 (m, arom. C-H), 137.7, 137.9,137.9, 138.2, 138.3, 138.6 (6q, arom. C- $\mathrm{CH}_{2} \mathrm{O}$ ), 168.9 (s, Ac); IR (Film) : v = 3031, $2895,1694,1554,1497,1392,1362,1244,1075,910,744,685,556 \mathrm{~cm}^{-1}$; elemental analysis cald (\%) for $\mathrm{C}_{58} \mathrm{H}_{65} \mathrm{NO}_{11}(952)$ : C 73.16, H 6.88, N 1.47; found: $\mathrm{C} 73.24, \mathrm{H} 7.01, \mathrm{~N} 1.64$.

\section{Methyl 2-acetamidomethyl-3,6,8,9,10,12-hexa- $O$-benzyl-2-deoxy- $\beta$-D-lactopyranoside} (7g):

$\mathrm{R}_{\mathrm{f}}=0.74$ (ethyl acetate /methanol 9:1); $[\alpha]_{\mathrm{D}}{ }^{20}=+13.2\left(\mathrm{c}=1.00\right.$ in $\left.\mathrm{CHCl}_{3}\right) ;{ }^{1} \mathrm{H}-\mathrm{NMR}(500$ $\mathrm{MHz}, \mathrm{CDCl}_{3}$ ): $\delta=1.64$ (s, $3 \mathrm{H}, \mathrm{Ac}$ ), 1.71 (dddd, $\mathrm{J}=10.4,8.6,5.5,4.2 \mathrm{~Hz}, 1 \mathrm{H}, 2-\mathrm{H}$ ), 3.25 (ddd, J = 7.4, 5.2, $2.2 \mathrm{~Hz}, 1 \mathrm{H}, 5-\mathrm{H}), 3.29$ (dd, J = 7.4, $2.0 \mathrm{~Hz}, 1 \mathrm{H}, 4-\mathrm{H}), 3.34$ (ddd, J = 8.8, 4.4, 1.4 Hz, $1 \mathrm{H}, 11-\mathrm{H}), 3.40$ (dd, J = 8.8, $4.0 \mathrm{~Hz}, 1 \mathrm{H}, 10-\mathrm{H}), 3.41$ (s, $3 \mathrm{H}, \mathrm{OMe}), 3.62$ (dd, J $=10.7,1.4 \mathrm{~Hz}, 1 \mathrm{H}, 12-\mathrm{H}), 3.63(\mathrm{dd}, \mathrm{J}=11.0,4.2 \mathrm{~Hz}, 1 \mathrm{H}, 13-\mathrm{H}), 3.65$ (dd, J = 11.0, $5.5 \mathrm{~Hz}$, $1 \mathrm{H}, 13$ '-H), 3.67 (dd, J = 10.0, $\left.4.4 \mathrm{~Hz}, 1 \mathrm{H}, 12^{\prime}-\mathrm{H}\right), 3.79$ (dd, J = 11.0, $\left.5.2 \mathrm{~Hz}, 1-\mathrm{H}, 6-\mathrm{H}\right)$, $3.81(\mathrm{dd}, \mathrm{J}=11.0,2.2 \mathrm{~Hz}, 1-\mathrm{H}, 6$ '-H), 3.82 (dd, J = 9.1, 4.0 Hz, $1 \mathrm{H}, 9-\mathrm{H}), 3.96$ (dd, J = 10.6, $9.1 \mathrm{~Hz}, 1 \mathrm{H}, \mathrm{H}-8), 4.02$ (dd, J = 10.4, $2.0 \mathrm{~Hz}, 1 \mathrm{H}, \mathrm{H}-3), 4.04(\mathrm{~d}, \mathrm{~J}=8.6 \mathrm{~Hz}, 1 \mathrm{H}, 1-\mathrm{H}), 4.18$ $\left(\mathrm{d}, \mathrm{J}=11.5 \mathrm{~Hz}, 1 \mathrm{H}, \mathrm{CH}_{2}-\mathrm{Ph}\right), 4.20\left(\mathrm{~d}, \mathrm{~J}=12.0 \mathrm{~Hz}, 1 \mathrm{H}, \mathrm{CH}_{2}-\mathrm{Ph}\right), 4,29$ (d, J = $12.0 \mathrm{~Hz}, 1 \mathrm{H}$, $\left.\mathrm{CH}_{2}-\mathrm{Ph}\right), 4.37$ (d, J = 11.4, Hz, $\left.1 \mathrm{H}, \mathrm{CH}_{2}-\mathrm{Ph}\right), 4.38$ (d, J = 12.0 Hz, $\left.1 \mathrm{H}, \mathrm{CH}_{2}-\mathrm{Ph}\right), 4.45$ (d, J = $11.4 \mathrm{~Hz}, 1 \mathrm{H}, \mathrm{CH}_{2}-\mathrm{Ph}$ ), 4.48 (d, J = 12.0 Hz, $1 \mathrm{H}, \mathrm{CH}_{2}-\mathrm{Ph}$ ), 4.50 (d, J = $12.0 \mathrm{~Hz}, 1 \mathrm{H}, \mathrm{CH}_{2}-$ $\mathrm{Ph}), 4.64\left(\mathrm{~d}, \mathrm{~J}=12.0 \mathrm{~Hz}, 1 \mathrm{H}, \mathrm{CH}_{2}-\mathrm{Ph}\right), 4.71$ (d, J = 11.4 Hz, $\left.1 \mathrm{H}, \mathrm{CH}_{2}-\mathrm{Ph}\right), 4.78$ (d, J = 11.4 $\left.\mathrm{Hz}, 1 \mathrm{H}, \mathrm{CH}_{2}-\mathrm{Ph}\right), 4.87$ (d, J = 11.4 Hz, $\left.1 \mathrm{H}, \mathrm{CH}_{2}-\mathrm{Ph}\right), 5.07$ (d, J = 10.6 Hz, $\left.1 \mathrm{H}, 7-\mathrm{H}\right), 5.86$ (t, $\mathrm{J}=4.7 \mathrm{~Hz}, 1 \mathrm{H}, \mathrm{NH}), 7.10-7.30(\mathrm{~m}, 30 \mathrm{H}$, arom. $\mathrm{H}) ;{ }^{13} \mathrm{C}-\mathrm{NMR}\left(75 \mathrm{MHz}, \mathrm{CDCl}_{3}\right): \delta=23.0$ (q, Ac), 38.2 (t, $\left.\mathrm{CH}_{2}-\mathrm{NH}\right), 45.0$ (d, C-2), 56.5 (q, OMe), 68.5, 69.6 (2t, C-6, C-12) 71.1 (d, C3), 73.3 , 73.2, 73.4 (t, $\left.\mathrm{CH}_{2}-\mathrm{Ph}\right), 74.7$ (d, C-4), 75.0 (t, $\left.\mathrm{CH}_{2}-\mathrm{Ph}\right), 75.3$ (d, C-5), 75.4 (t, $\mathrm{CH}_{2}-$ $\mathrm{Ph}), 77.7,79.7,80.0,81.8,91.7,103.1$ (6d, C-8, C-9, C-10, C-11, C-7, C-1), 127.5, 127.6, $127.6,127.7,127.8,127.8,128.0,128.0,128.2$, 128.3, 128.3, 128.5 (m, arom, C-H), 137.7, 
137.9,137.9, 138.2, 138.3, 138.6 (6q, arom. C- $\mathrm{CH}_{2} \mathrm{O}$ ), 168.9 (s, Ac); IR (Film) : v = 3028, $2862,1722,1652,1496,1452,1362,1273,1065,910,732,695,596 \mathrm{~cm}^{-1}$; elemental analysis cald (\%) for $\mathrm{C}_{58} \mathrm{H}_{65} \mathrm{NO}_{11}(952): \mathrm{C}: 73.16, \mathrm{H}: 6.88, \mathrm{~N}: 1.47$; found: $\mathrm{C}: 72.97, \mathrm{H}: 6.71, \mathrm{~N}$ $: 1.42$.

\section{Catalytic hydrogenation of the nitro group (method E)}

The 2-deoxy-2-C-nitromethyl-pyranoside 4 ( $1 \mathrm{mmol})$ was dissolved in methanol $(5 \mathrm{~mL})$ and palladium on carbon $(10 \%)(120 \mathrm{mg})$ was added. The reaction mixture was stirred in an autoclave under $\mathrm{H}_{2}$ atmosphere (40 bar) for $1 \mathrm{~h}$ and TLC showed complete conversion. The mixture was filtered over celite, concentrated and acetylated with acetic anhydride $(0.5 \mathrm{~mL})$ for $30 \mathrm{~min}$. After concentration in vacuum, the crude product was purified by column chromatography (ethyl acetate/ methanol $8: 2$ ) to afford the branched-chain glycosamines 1 in analytically pure form.

\section{Methyl 2-acetamidomethyl-2-deoxy- $\beta$-D-glucopyranoside (1b):}

$\mathrm{R}_{\mathrm{f}}=0.50$ (ethyl acetate/ methanol 8:2); $[\alpha]_{\mathrm{D}}{ }^{20}=-8.1\left(\mathrm{c}=0.99\right.$ in $\left.\mathrm{H}_{2} \mathrm{O}\right) ;{ }^{1} \mathrm{H}-\mathrm{NMR}(500 \mathrm{MHz}$, $\left.\mathrm{D}_{2} \mathrm{O}\right): \delta=1.51$ (dddd, $\left.\mathrm{J}=10.6,8.8,5.6,3.2 \mathrm{~Hz}, 1 \mathrm{H}, 2-\mathrm{H}\right), 1.91(\mathrm{~s}, 3 \mathrm{H}, \mathrm{Ac}), 3.23$ (ddd, J = 9.9, 5.6, 1.5 Hz, 1 H, 5-H), 3.27 (dd, J = 9.9, 9.5 Hz, $1 \mathrm{H}, 4-\mathrm{H}), 3.29$ (dd, J = 14.4, $5.6 \mathrm{~Hz}, 1$ H, 7-H), 3.37 (dd, J = 10.6, 9.5 Hz, 1 H, 3-H), 3.43 (s, 3 H, OMe), 3.47 (dd, J = 14.4, 3.2 Hz, $\left.1 \mathrm{H}, 7^{\prime}-\mathrm{H}\right), 3.62$ (dd, J = 12.2, $\left.5.6 \mathrm{~Hz}, 1 \mathrm{H}, 6-\mathrm{H}\right), 3.82$ (dd, J =12.2, $1.5 \mathrm{~Hz}, 1 \mathrm{H}, 6$ '-H), 4.29 $(\mathrm{d}, \mathrm{J}=8.8 \mathrm{~Hz}, 1 \mathrm{H}, 1-\mathrm{H}), 4.69(\mathrm{~s}, 3 \mathrm{H}, \mathrm{OH}) ;{ }^{13} \mathrm{C}-\mathrm{NMR}\left(75 \mathrm{MHz}, \mathrm{D}_{2} \mathrm{O}\right): \delta=22.3(\mathrm{q}, \mathrm{Ac}), 36.4$ (t, $\left.\mathrm{CH}_{2}-\mathrm{NH}\right), 48.0$ (d, C-2), 57.4 (q, OMe), 61.4 (t, C-6), 71.1, 72.5, 76.1, 103.2 (4d, C-3, C-4, C-5, C-1), 174.6 (s, Ac); IR (Film) : v = 3276, 2867, 1627, 1552, 1451, 1370, 1210, 1069, 1024, 733, 696, 604, $573 \mathrm{~cm}^{-1}$; HR-MS(ES) $\left(\mathrm{C}_{10} \mathrm{H}_{19} \mathrm{NO}_{6}\right)$ : calcd for [M+Na] 272.1110; found 272.1128 .

\section{Methyl 2-acetamidomethyl-2-deoxy- $\beta$-D-galactopyranoside (1c):}

$\mathrm{R}_{\mathrm{f}}=0.44$ (ethyl acetate/ methanol 8:2); $[\alpha]_{\mathrm{D}}{ }^{20}=-4.9\left(\mathrm{c}=1.00\right.$ in $\left.\mathrm{H}_{2} \mathrm{O}\right) ;{ }^{1} \mathrm{H}-\mathrm{NMR}(500 \mathrm{MHz}$, $\left.\mathrm{D}_{2} \mathrm{O}\right): \delta=1.80$ (dddd, $\left.\mathrm{J}=11.6,8.8,5.5,3.2 \mathrm{~Hz}, 1 \mathrm{H}, 2-\mathrm{H}\right), 1.90$ (s, $3 \mathrm{H}, \mathrm{Ac}$ ), 3.25 (dd, $\mathrm{J}=$ 12.6, $5.5 \mathrm{~Hz}, 1 \mathrm{H}, 7-\mathrm{H}), 3.43$ (s, $3 \mathrm{H}, \mathrm{OMe}$ ), 3.45 (ddd, J = 11.8, 4.8, $2.0 \mathrm{~Hz}, 1 \mathrm{H}, 5-\mathrm{H}), 3.48$ (dd, J = 12.6, 3.2 Hz, 1 H, 7'-H), 3.44 (dd, J = 11.6, 4.7 Hz, 1 H, 3-H), 3.53 (dd, J = 11.3, 2.0 Hz, 1 H, 6-H), 3.64 (dd, J = 11.3, 4.8 Hz, 1 H, 6'-H), 3.72 (dd, J = 11.8, 4.7 Hz, 1 H, 4-H), $4.22(\mathrm{~d}, \mathrm{~J}=8.8 \mathrm{~Hz}, 1 \mathrm{H}, 1-\mathrm{H}) 4.69(\mathrm{~s}, 3 \mathrm{H}, \mathrm{OH}) ;{ }^{13} \mathrm{C}-\mathrm{NMR}\left(75 \mathrm{MHz}, \mathrm{D}_{2} \mathrm{O}\right): \delta=22.3(\mathrm{q}, \mathrm{Ac})$, 
36.4 (t, $\mathrm{CH}_{2}-\mathrm{NH}$ ), 43.2 (d, C-2), 57.4 (q, OMe), 61.7 (t, C-6), 67.8, 69.7, 75.3, 103.7 (4d, C-3, C-4, C-5, C-1), 174.6 (s, Ac); IR (Film) : $v=3304,2936,1735,1633,1556,1371,1234$, 1032, 776, $588 \mathrm{~cm}^{-1}$; HR-MS(ES) $\left(\mathrm{C}_{10} \mathrm{H}_{19} \mathrm{NO}_{6}\right)$ : calcd for [M+Na] 272.1110; found 272.1120.

\section{Methyl 2-acetamidomethyl-2-deoxy- $\beta$-D-xylopyranoside (1d):}

$\mathrm{R}_{\mathrm{f}}=0.60$ (ethyl acetate/ methanol 8:2); $[\alpha]_{\mathrm{D}}{ }^{20}=-20.1\left(\mathrm{c}=1.00\right.$ in $\left.\mathrm{H}_{2} \mathrm{O}\right) ;{ }^{1} \mathrm{H}-\mathrm{NMR}(500 \mathrm{MHz}$, $\left.\mathrm{D}_{2} \mathrm{O}\right): \delta=1.60$ (dddd, $\left.\mathrm{J}=10.8,8.7,5.5,3.3 \mathrm{~Hz}, 1 \mathrm{H}, 2-\mathrm{H}\right), 1.90$ (s, $\left.3 \mathrm{H}, \mathrm{Ac}\right), 3.16$ (dd, J = 11.5, 3.3 Hz, 1 H, 6-H), 3.26 (dd, J = 11.5, 5.5 Hz, 1 H, 6'-H), 3.30 (dd, J = 10.8, 8.9 Hz, 1 H, 3-H), 3.39 (s, 3 H, OMe), 3.47 (dd, J = 8.9, 5.5, 2.8 Hz, 1 H, 4-H), 3.50 (dd, J = 11.6, 2.8 Hz, $1 \mathrm{H}, 5-\mathrm{H}), 3.86\left(\mathrm{dd}, \mathrm{J}=11.6,5.5 \mathrm{~Hz}, 1 \mathrm{H}, 5^{\prime}-\mathrm{H}\right), 4.25$ (d, J = 8.7 Hz, $\left.1 \mathrm{H}, \mathrm{H}-1\right), 4.69$ (s, $2 \mathrm{H}$, $\mathrm{OH}) ;{ }^{13} \mathrm{C}-\mathrm{NMR}\left(75 \mathrm{MHz}, \mathrm{D}_{2} \mathrm{O}\right): \delta=22.3$ (q, Ac), $36.4\left(\mathrm{t}, \mathrm{CH}_{2}-\mathrm{NH}\right), 47.5$ (d, C-2), 57.3 (q, OMe), 65.2 (t, C-5), 70.5, 72.2, 103.8 (3d, C-3, C-4, C-1), 174.6 (s, Ac); IR (Film) : v = 3296, 2913, 1626, 1552, 1437, 1297, 1214, 1155, 1043, 1001, 943, 639, $599 \mathrm{~cm}^{-1}$; HR-MS(ES) $\left(\mathrm{C}_{9} \mathrm{H}_{17} \mathrm{NO}_{5}\right)$ : calcd for [M+Na] 242.1004; found 242.1016; elemental analysis cald (\%) for $\mathrm{C}_{9} \mathrm{H}_{17} \mathrm{NO}_{5}$ (219) : C 49.31, H 7.82, N 6.39; found: C 49.15, H 7.78, N 6.14.

\section{Methyl 2-acetamidomethyl-2-deoxy- $\alpha$-D-arabinopyranoside (1d):}

$\mathrm{R}_{\mathrm{f}}=0.53$ (ethyl acetate/ methanol 8:2); $[\alpha]_{\mathrm{D}}{ }^{20}=-4.2\left(\mathrm{c}=1.00\right.$ in $\left.\mathrm{H}_{2} \mathrm{O}\right) ;{ }^{1} \mathrm{H}-\mathrm{NMR}(500 \mathrm{MHz}$, $\left.\mathrm{D}_{2} \mathrm{O}\right): \delta=1.77$ (dddd, $\left.\mathrm{J}=10.2,8.6,5.8,3.4 \mathrm{~Hz}, 1 \mathrm{H}, 2-\mathrm{H}\right), 1.90(\mathrm{~s}, 3 \mathrm{H}, \mathrm{Ac}), 3.25$ (dd, J = 14.3, 5.8 Hz, 1 H, 6-H), 3.41 (s, 3 H, OMe), 3.46 (dd, J = 14.3, 3.4 Hz, 1 H, 6'-H), 3.52 (dd, J $=12.7,1.1 \mathrm{~Hz}, 1 \mathrm{H}, 5-\mathrm{H}), 3.58(\mathrm{dd}, \mathrm{J}=10.2,3.3 \mathrm{~Hz}, 1 \mathrm{H}, 3-\mathrm{H}), 3.75(\mathrm{dd}, \mathrm{J}=3.3,2.8,1.1 \mathrm{~Hz}$, $1 \mathrm{H}, 4-\mathrm{H}), 3.83$ (dd, J = 12.7, $\left.2.8 \mathrm{~Hz}, 1 \mathrm{H}, 5^{\prime}-\mathrm{H}\right), 4.20$ (d, J = 8.6 Hz, $\left.1 \mathrm{H}, 1-\mathrm{H}\right), 4.69$ (s, 2 H, $\mathrm{OH}) ;{ }^{13} \mathrm{C}-\mathrm{NMR}\left(75 \mathrm{MHz}, \mathrm{D}_{2} \mathrm{O}\right): \delta=22.3(\mathrm{q}, \mathrm{Ac}), 36.6\left(\mathrm{t}, \mathrm{CH}_{2}-\mathrm{NH}\right), 43.6(\mathrm{~d}, \mathrm{C}-2), 57.2(\mathrm{q}$, OMe), 66.0 (t, C-5), 67.2, 69.1, 103.6 (3d, C-3, C-4, C-1), 174.8 (s, Ac); IR (Film) : v = 3324, 2943, 1654, 1492, 1417, 1287, 1153, 1049, 967, $587 \mathrm{~cm}^{-1}$; HR-MS(ES) $\left(\mathrm{C}_{9} \mathrm{H}_{17} \mathrm{NO}_{5}\right)$ : calcd for $[M+\mathrm{Na}]$ 242.1004; found 242.1002.

\section{Methyl 2-acetamidomethyl-2-deoxy- $\beta$-D-maltopyranoside (1f):}

$\mathrm{R}_{\mathrm{f}}=0.54$ (ethyl acetate/ methanol 7:3); $[\alpha]_{\mathrm{D}}{ }^{20}=-14.2\left(\mathrm{c}=1.01\right.$ in $\left.\mathrm{H}_{2} \mathrm{O}\right) ;{ }^{1} \mathrm{H}-\mathrm{NMR}(500$ $\mathrm{MHz}, \mathrm{D}_{2} \mathrm{O}$ ): $\delta=1.67$ (dddd, J = 10.8, 8.6, 5.4, 3.2 Hz, $\left.1 \mathrm{H}, 2-\mathrm{H}\right), 2.02$ (s, $3 \mathrm{H}, \mathrm{Ac}$ ), 3.35 (dd, J $=11.6,5.4 \mathrm{~Hz}, 1 \mathrm{H}, 13-\mathrm{H}), 3.37$ (dd, J = 11.6, $\left.3.2 \mathrm{~Hz}, 1 \mathrm{H}, 13^{\prime}-\mathrm{H}\right), 3.38$ (dd, J = 11.4, $5.9 \mathrm{~Hz}$, $1 \mathrm{H}, 12-\mathrm{H}), 3.43$ (ddd, J = 9.3, 5.4, 3.3 Hz, 1 H, 5-H), 3.51 (dd, J = 11.4, 2.4 Hz, 1 H, 12'-H), 3,54 (s, 3 H, OMe), 3.57 (ddd, J = 10.7, 5.9, 2.4 Hz, $1 \mathrm{H}, 11-\mathrm{H}), 3.59$ (dd, J = 9.3, 8.7 Hz, 1 H, 4-H), 3.62 (dd, J = 10.7, 9.2 Hz, 1 H, 10-H), 3.69 (dd J = 10.8, 8.7 Hz, 1 H, 3-H), 3.72 (dd, 
$\mathrm{J}=12.0,5.4 \mathrm{~Hz}, 1 \mathrm{H}, 6-\mathrm{H}), 3.86(\mathrm{dd}, \mathrm{J}=9.3,8.6 \mathrm{~Hz}, 1 \mathrm{H} .9-\mathrm{H}), 3.89$ (dd, $\mathrm{J}=12.0,3.3 \mathrm{~Hz}, 1$ H, 6'-H), 3.97 (dd, J = 8.6, 3.5 Hz, 1 H, 8-H), 4.69 (s, 6 H, OH), 4.42 (d, J = 8.6 Hz, 1 H, 1-

$\mathrm{H}), 5.41(\mathrm{~d}, \mathrm{~J}=3.5 \mathrm{~Hz}, 1 \mathrm{H}, 7-\mathrm{H}) ;{ }^{13} \mathrm{C}-\mathrm{NMR}\left(75 \mathrm{MHz}, \mathrm{D}_{2} \mathrm{O}\right): \delta=22.3$ (q, Ac), $36.2\left(\mathrm{t}, \mathrm{CH}_{2}-\right.$ $\mathrm{NH}), 48.0$ (d, C-2), 57.4 (q, OMe), 61.0, 61.4 (2t, C-6, C-12), 69.8, 72.1, 73.0, 73.2, 73.3, 74.8, 78.7 (7d, C-3, C-4, C-5, C-8, C-9, C-10, C-11), 100.2, 103.1 (2d, C-1, C-7), 174.7 (s, Ac) ; IR (Film) : $v=3311,2925,2479,1624,1557,1427,1372,1244,1021,598,564 \mathrm{~cm}^{-1}$; HR-MS(ES) $\left(\mathrm{C}_{16} \mathrm{H}_{29} \mathrm{NO}_{11}\right)$ : calcd for $[M+\mathrm{Na}]$ 434.1638; found 434.1642.

\section{Methyl 2-acetamidomethyl-2-deoxy- $\beta$-D-lactopyranoside (1g):}

$\mathrm{R}_{\mathrm{f}}=0.59$ (ethyl acetate/ methanol 7:3); $[\alpha]_{\mathrm{D}}{ }^{20}=-8.5\left(\mathrm{c}=0.99\right.$ in $\left.\mathrm{H}_{2} \mathrm{O}\right) ;{ }^{1} \mathrm{H}-\mathrm{NMR}(500 \mathrm{MHz}$, $\mathrm{D}_{2} \mathrm{O}$ ): $\delta=1.53$ (dddd, $\left.\mathrm{J}=9.6,8.8,4.6,4.3 \mathrm{~Hz}, 1 \mathrm{H}, 2-\mathrm{H}\right), 1.89$ (s, $3 \mathrm{H}, \mathrm{Ac}$ ), 3.23 (dd, J = 11.1, 4.6 Hz, $1 \mathrm{H}, 13-\mathrm{H}), 3.28$ (dd, J = 12.2, $6.5 \mathrm{~Hz}, 1 \mathrm{H}, 12-\mathrm{H}), 3.42$ (s, OMe), 3.43 (dd, J = 11.1, $4.3 \mathrm{~Hz}, 1 \mathrm{H}, 13$ '-H), 3.46 (ddd, J = 8.8, 6.9, 3.5 Hz, $1 \mathrm{H}, 5-\mathrm{H}), 3.49$ (ddd, J = 9.6, 6.5, 3.7 Hz, $1 \mathrm{H}, 11-\mathrm{H}), 3.55$ (dd, J = 9.8, 3.2 Hz, $1 \mathrm{H}, 9-\mathrm{H}), 3.60$ (dd, J = 9.6, $3.4 \mathrm{~Hz}, 1 \mathrm{H}, 3-\mathrm{H}), 3.62$ (dd, $\mathrm{J}=8.4,3.4 \mathrm{~Hz}, 1 \mathrm{H}, 4-\mathrm{H}), 3.65(\mathrm{dd}, \mathrm{J}=12.4,3.5 \mathrm{~Hz}, 1 \mathrm{H}, 6-\mathrm{H}), 3.67$ (dd, J = 9.6, 3.2 Hz, $1 \mathrm{H}$, 10-H), 3.80-3.82 (m,1 H, H-8), 3.88 (dd, J = 12.2, 3.7 Hz, 1 H, 12'-H), 4.29 (d, J = 8.8 Hz, 1 H, 1-H), 4.34 (d, J = 7.7 Hz, 1 H, 7-H), 4.62 (dd, J = 12.4, 6.9 Hz, 1 H, 6'-H), 4.69 (s, 6 H, $\mathrm{OH}) ;{ }^{13} \mathrm{C}-\mathrm{NMR}\left(75 \mathrm{MHz}, \mathrm{D}_{2} \mathrm{O}\right): \delta=22.4(\mathrm{q}, \mathrm{Ac}), 36.4\left(\mathrm{t}, \mathrm{CH}_{2}-\mathrm{NH}\right), 47.5(\mathrm{~d}, \mathrm{C}-2), 57.5(\mathrm{q}$, OMe), 60.8, 61.4 (2t, C-6, C-12), 69.0, 71.2, 71.4, 73.0, 74.9, 75.8, 80.4 (7d, C-3, C-4, C-5, C-8, C-9, C-10, C-11), 103.0, 103.5 (2d, C-1, C-7), 174.4 (s, Ac); IR (Film) : v = 3369, 2845, 2494, 1627, 1425, 1373, 1072, 1030, 776, $592 \mathrm{~cm}^{-1}$; HR-MS(ES) $\left(\mathrm{C}_{16} \mathrm{H}_{29} \mathrm{NO}_{11}\right)$ : calcd for $[M+\mathrm{Na}]$ 434.1638; found 434.1662; elemental analysis cald (\%) for $\mathrm{C}_{16} \mathrm{H}_{29} \mathrm{NO}_{11}$ (411) : C 46.71, H 7.11, N 3.40; found: C 45.63, H 7.25, N 3.33. 


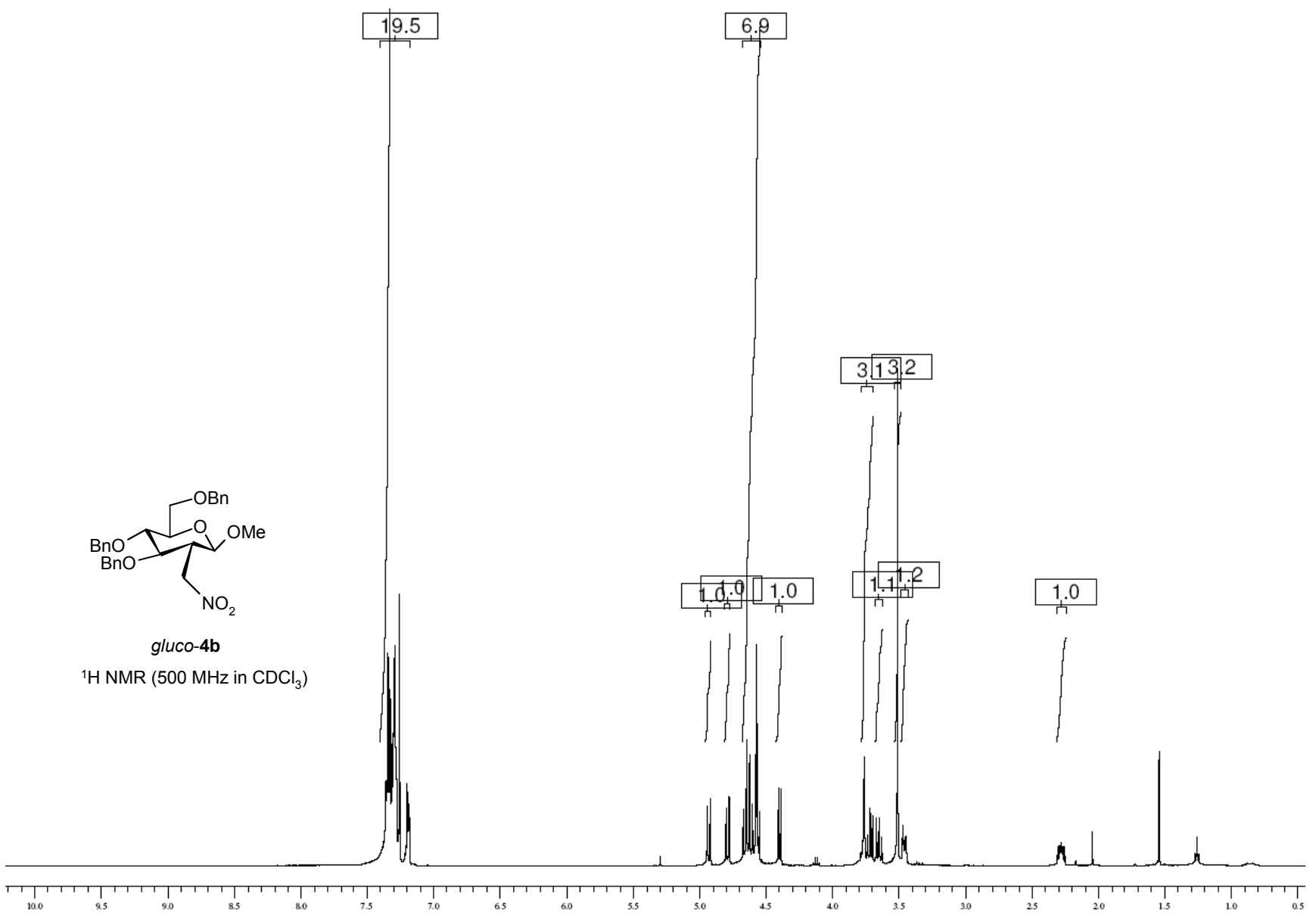

${ }^{13} \mathrm{C}$ NMR $\left(75 \mathrm{MHz}\right.$ in $\mathrm{CDCl}_{3}$ )
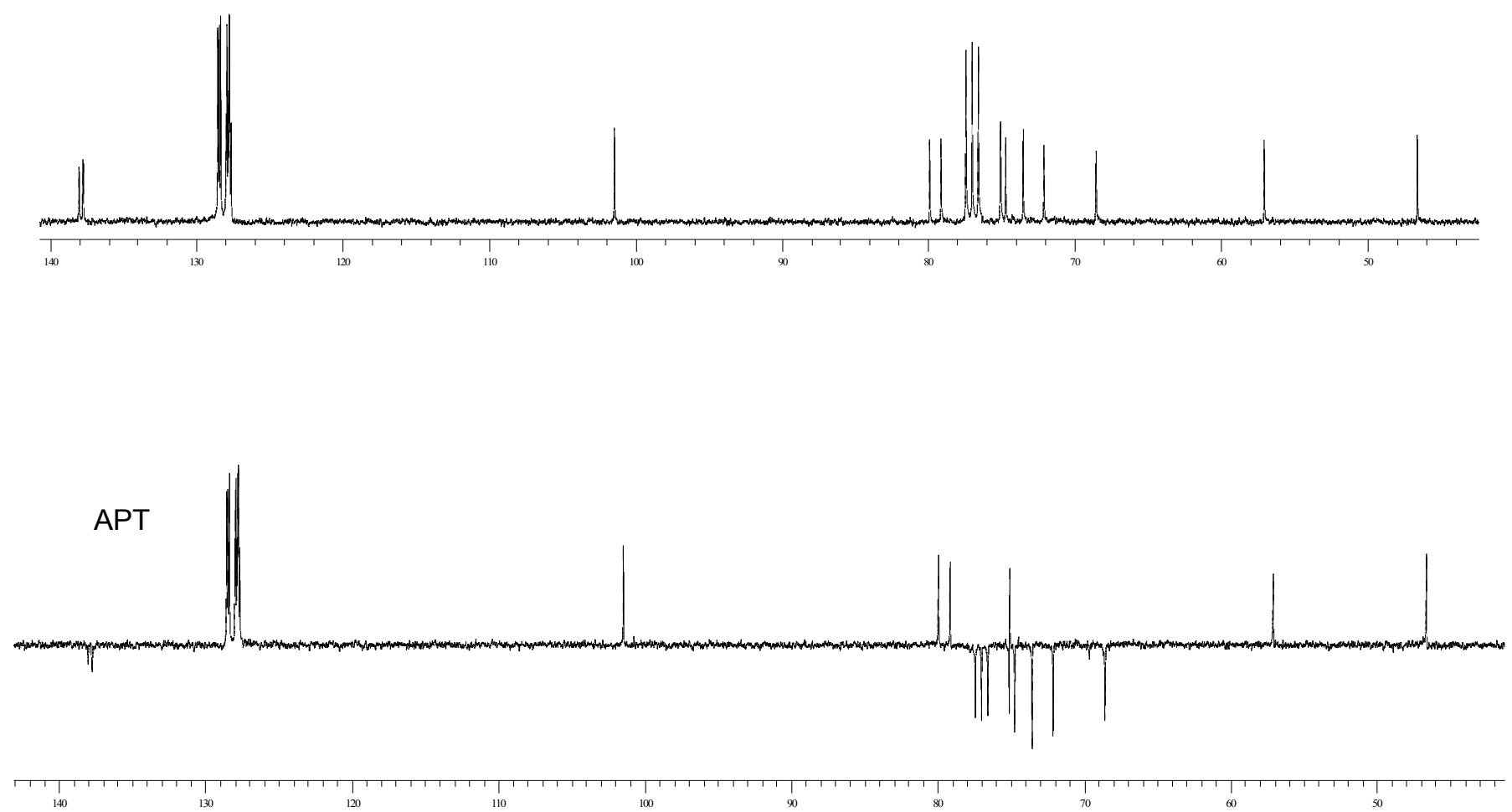
S16
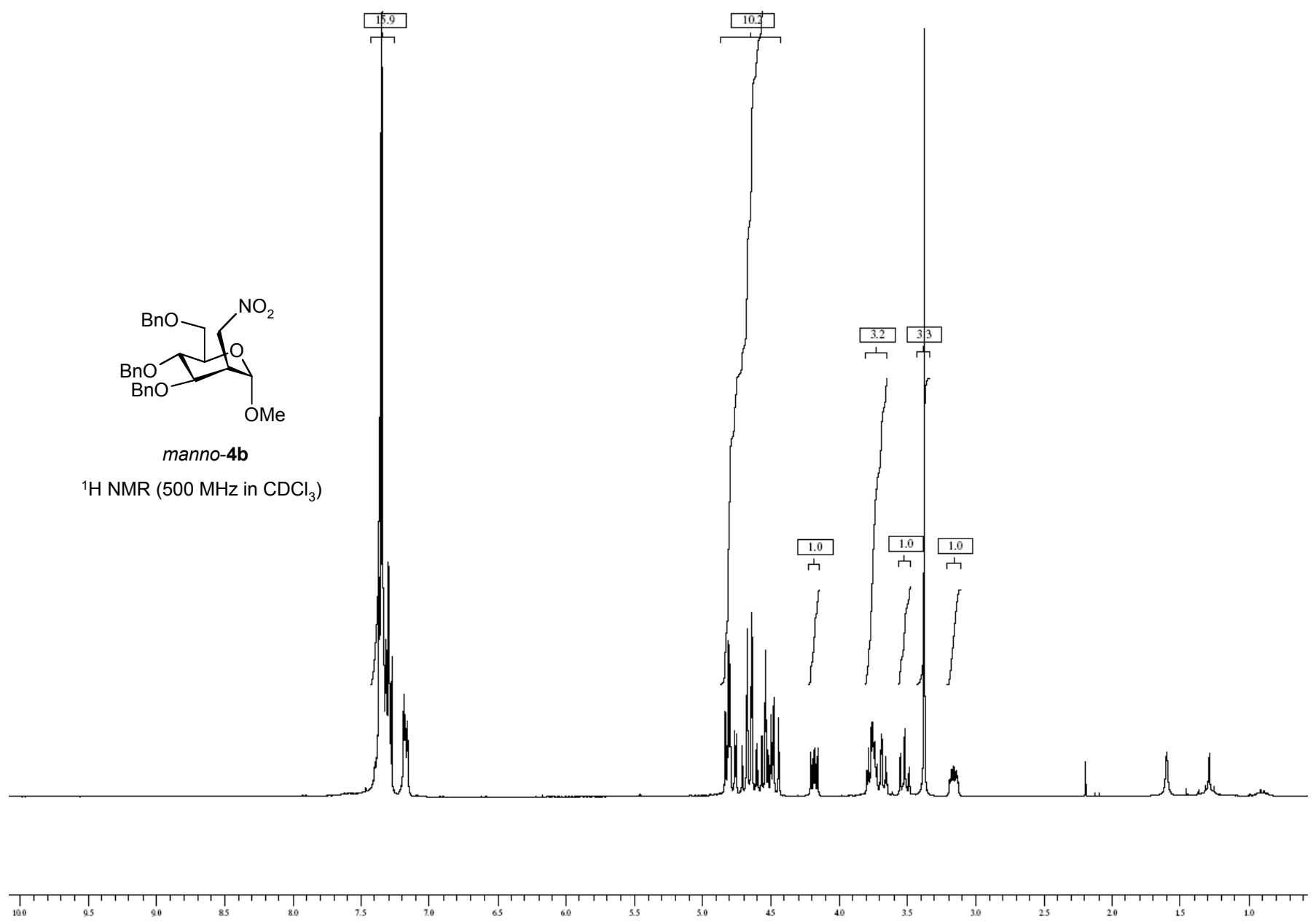

${ }^{13} \mathrm{C} \mathrm{NMR}\left(75 \mathrm{MHz}\right.$ in $\left.\mathrm{CDCl}_{3}\right)$
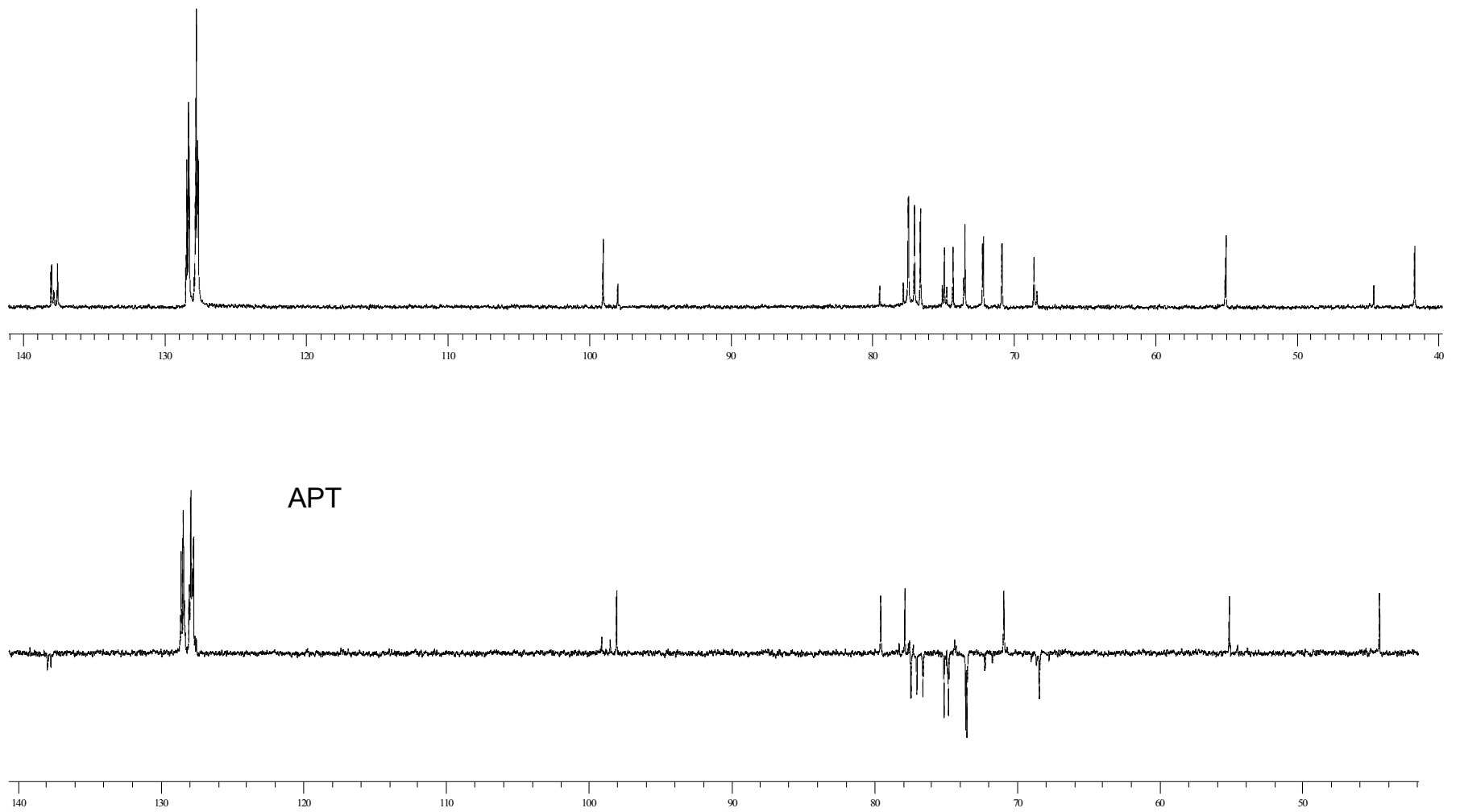


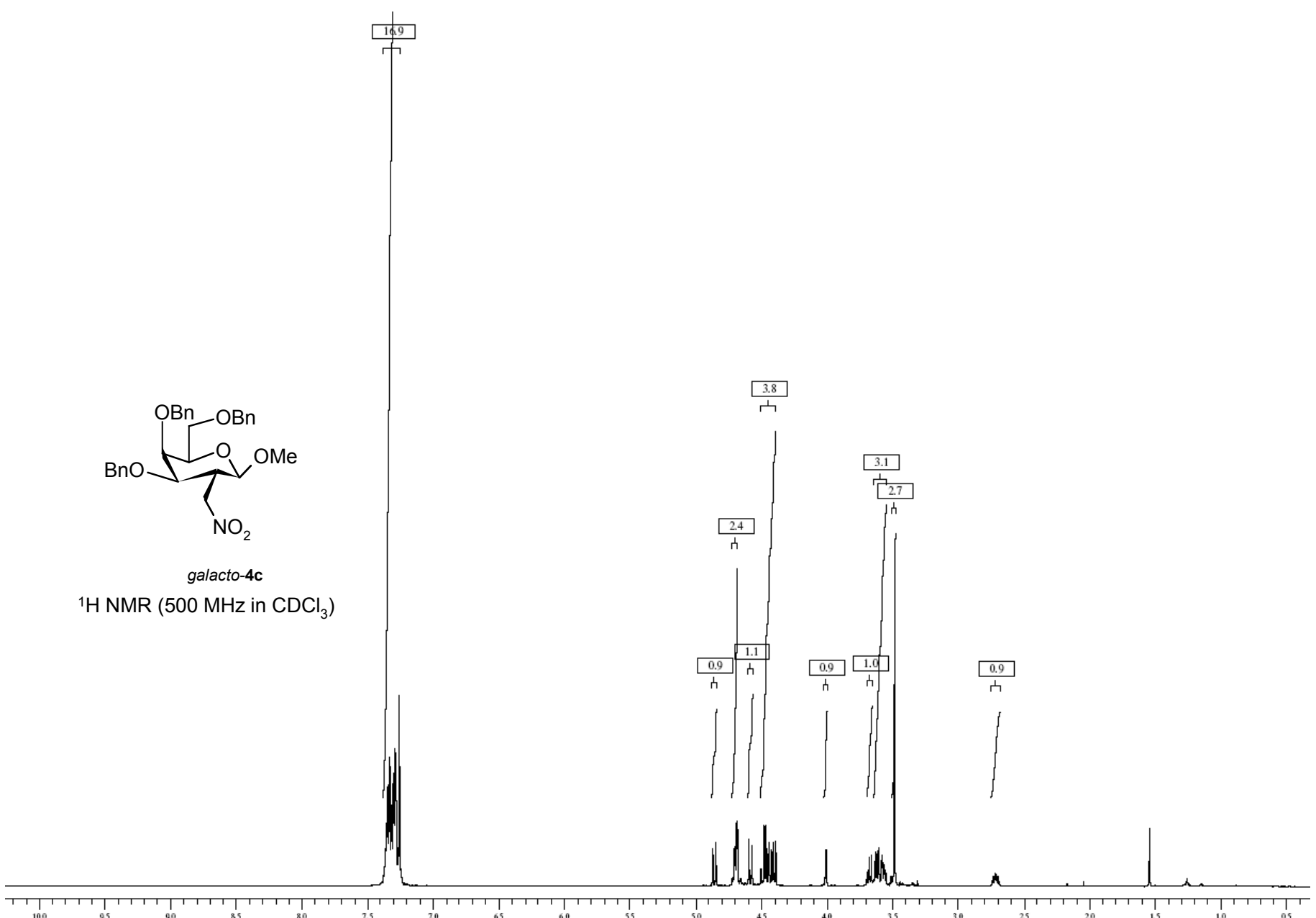

${ }^{13} \mathrm{C}$ NMR $\left(75 \mathrm{MHz}\right.$ in $\mathrm{CDCl}_{3}$ )
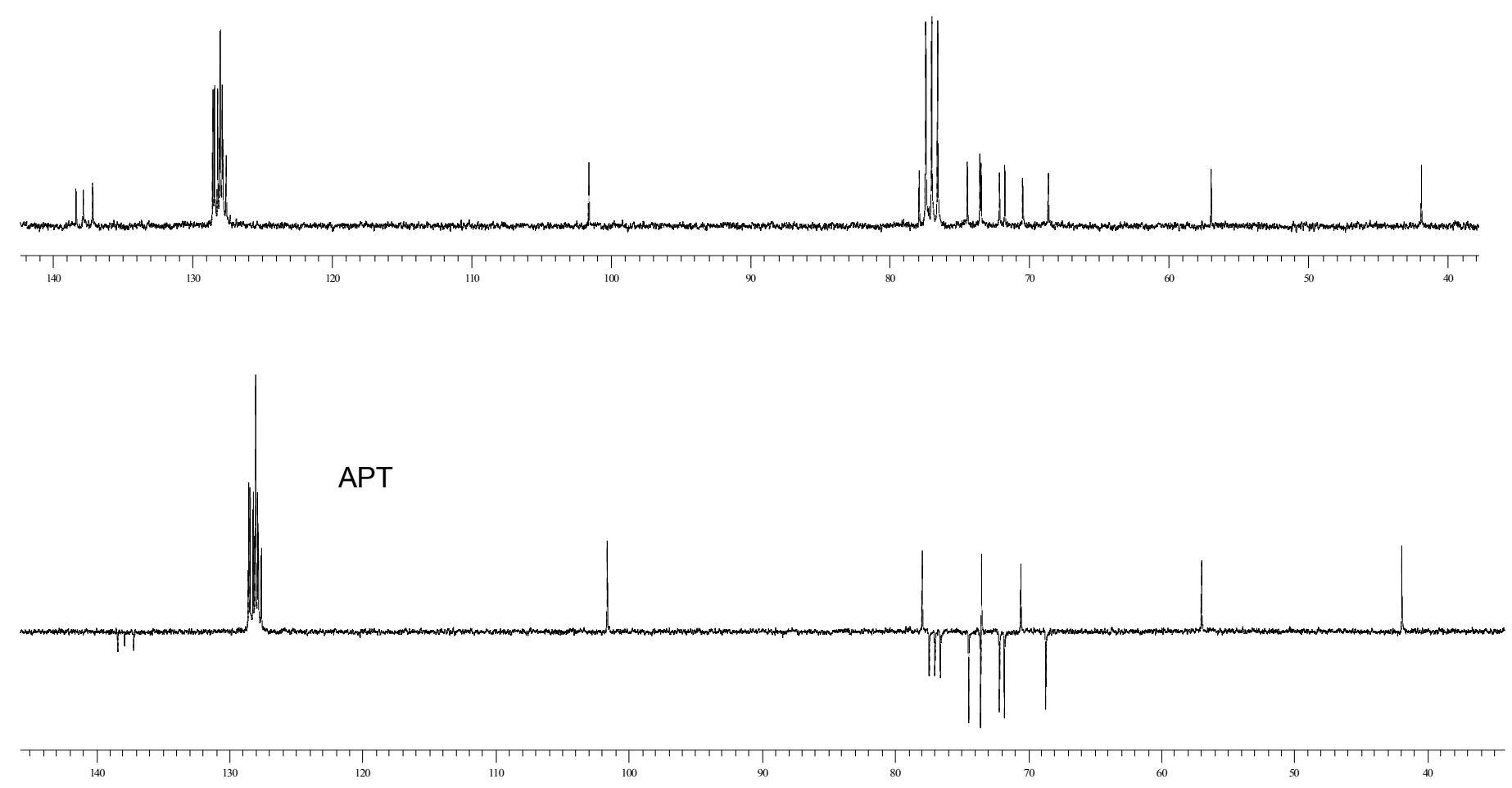

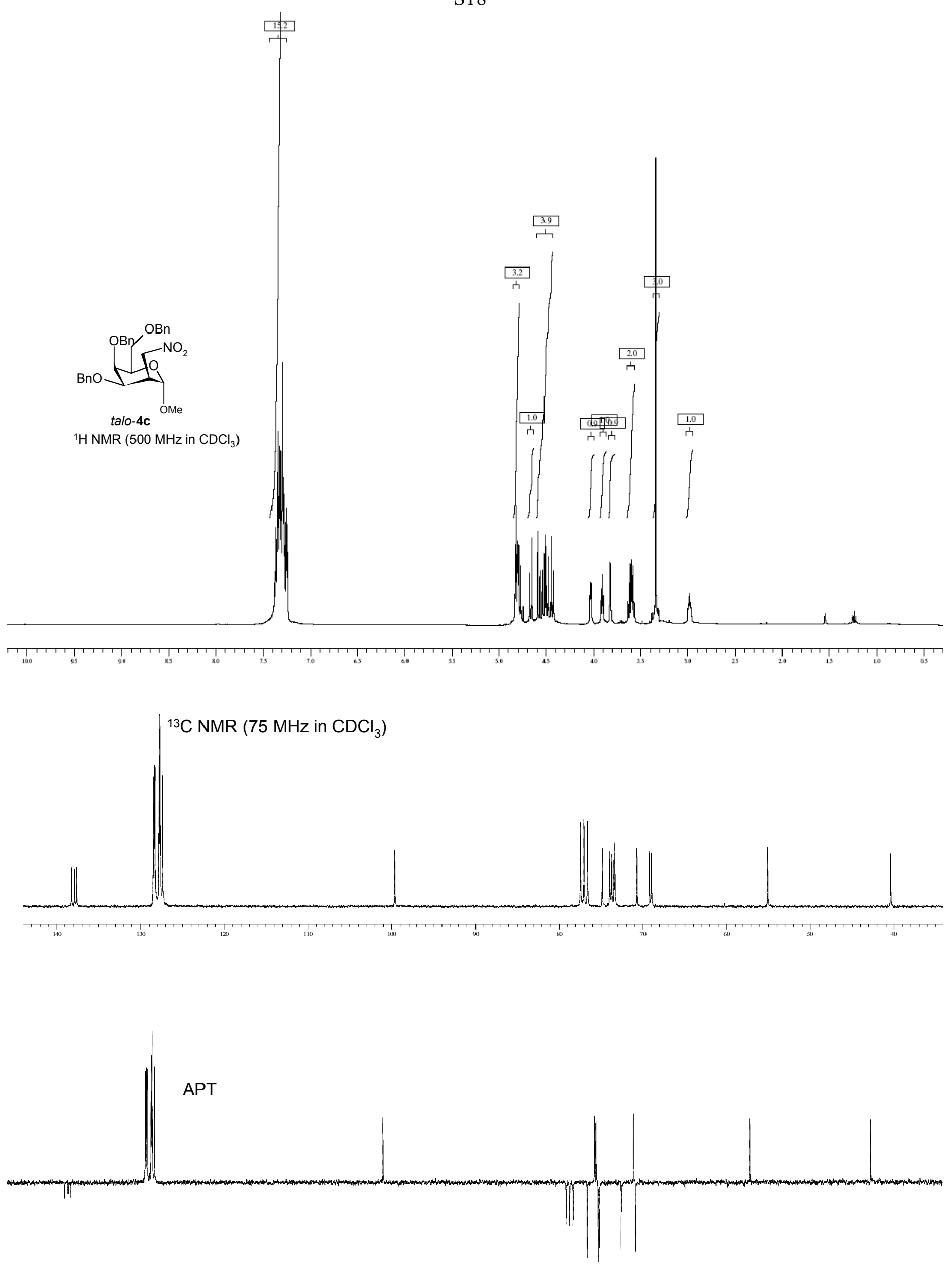


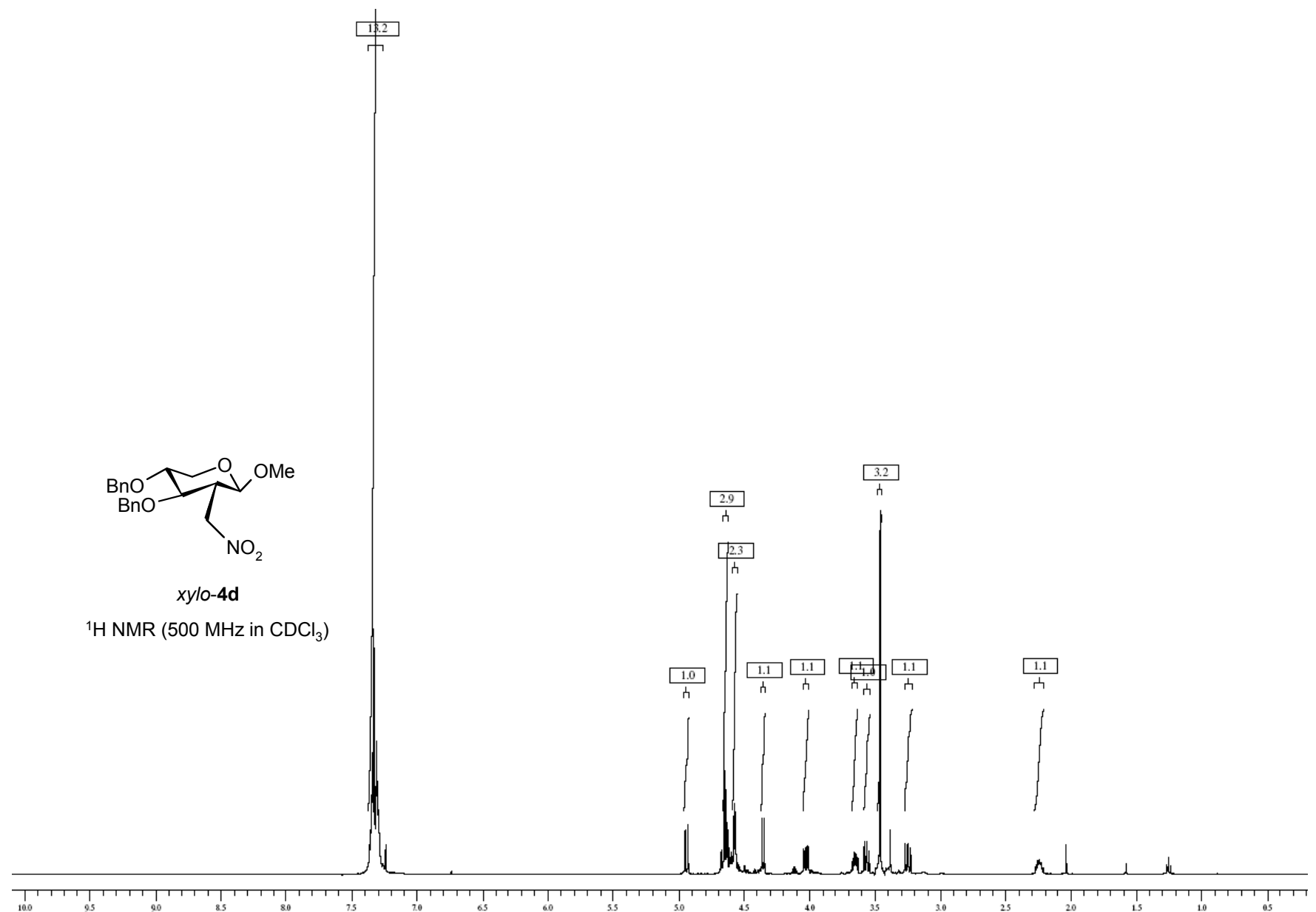

${ }^{13} \mathrm{C}$ NMR $\left(75 \mathrm{MHz}\right.$ in $\mathrm{CDCl}_{3}$ )
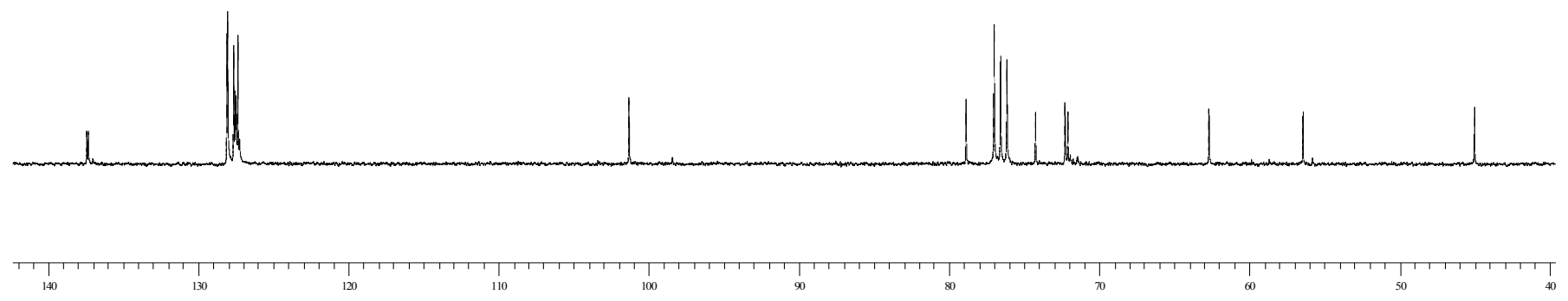

APT
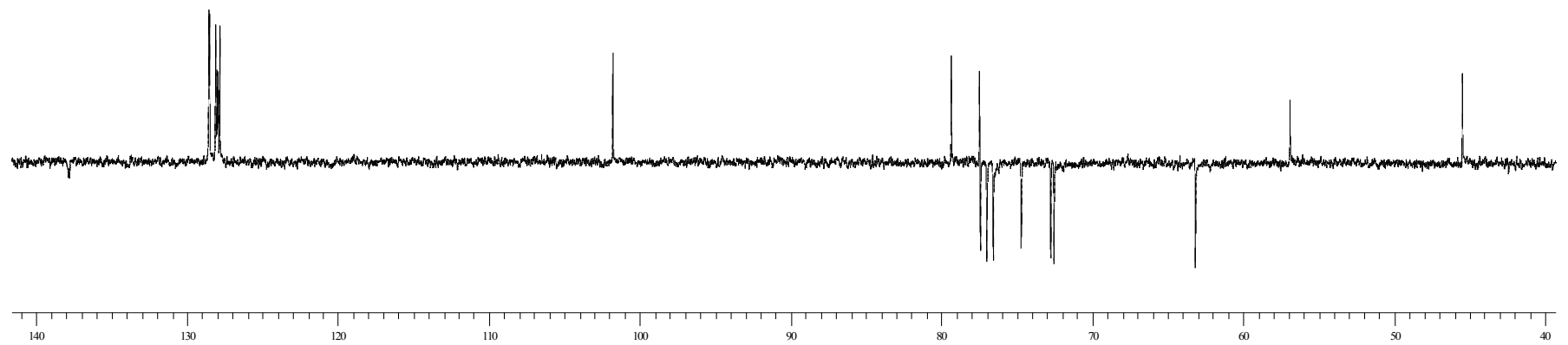

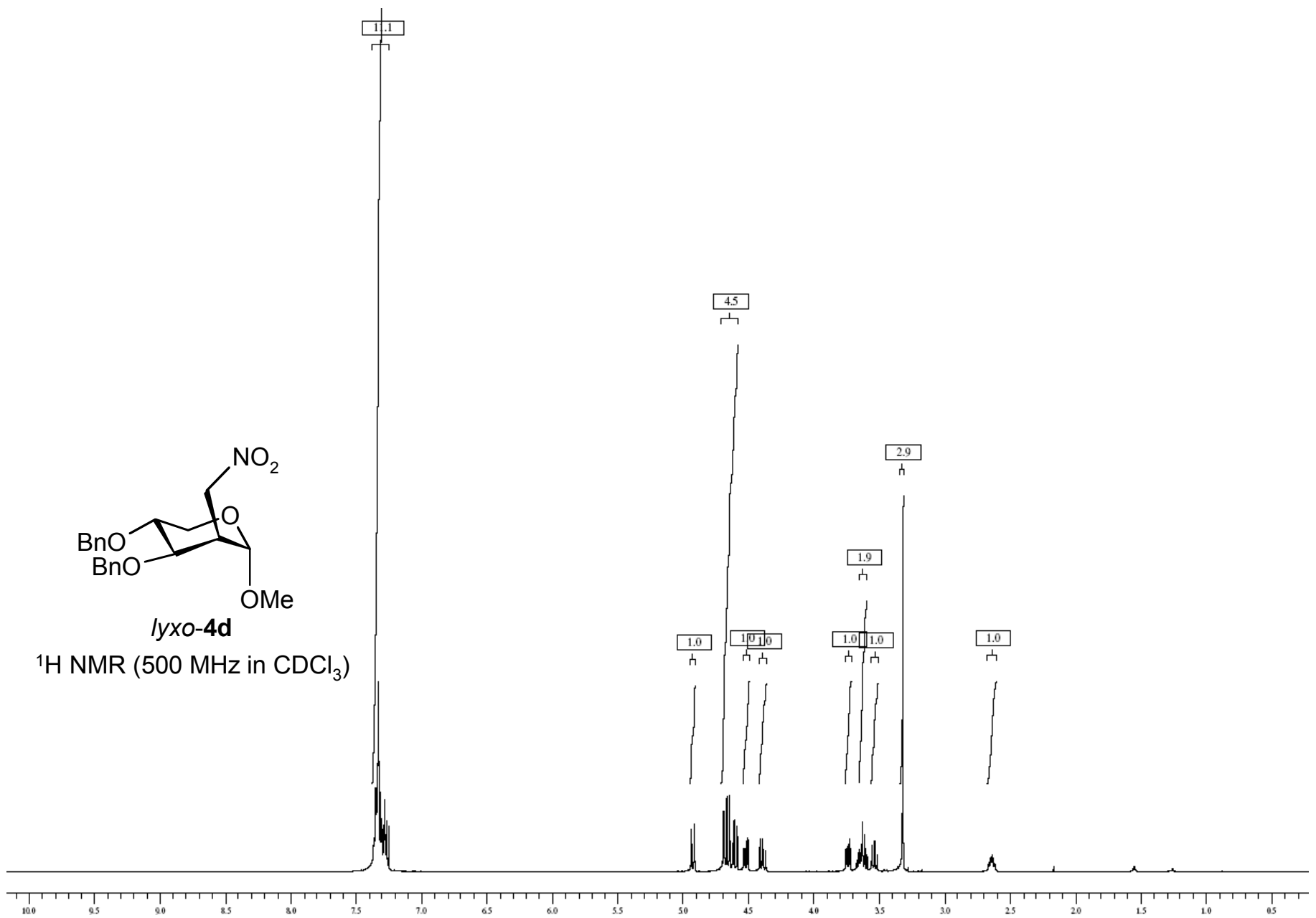

${ }^{13} \mathrm{C} \mathrm{NMR}\left(75 \mathrm{MHz}\right.$ in $\left.\mathrm{CDCl}_{3}\right)$
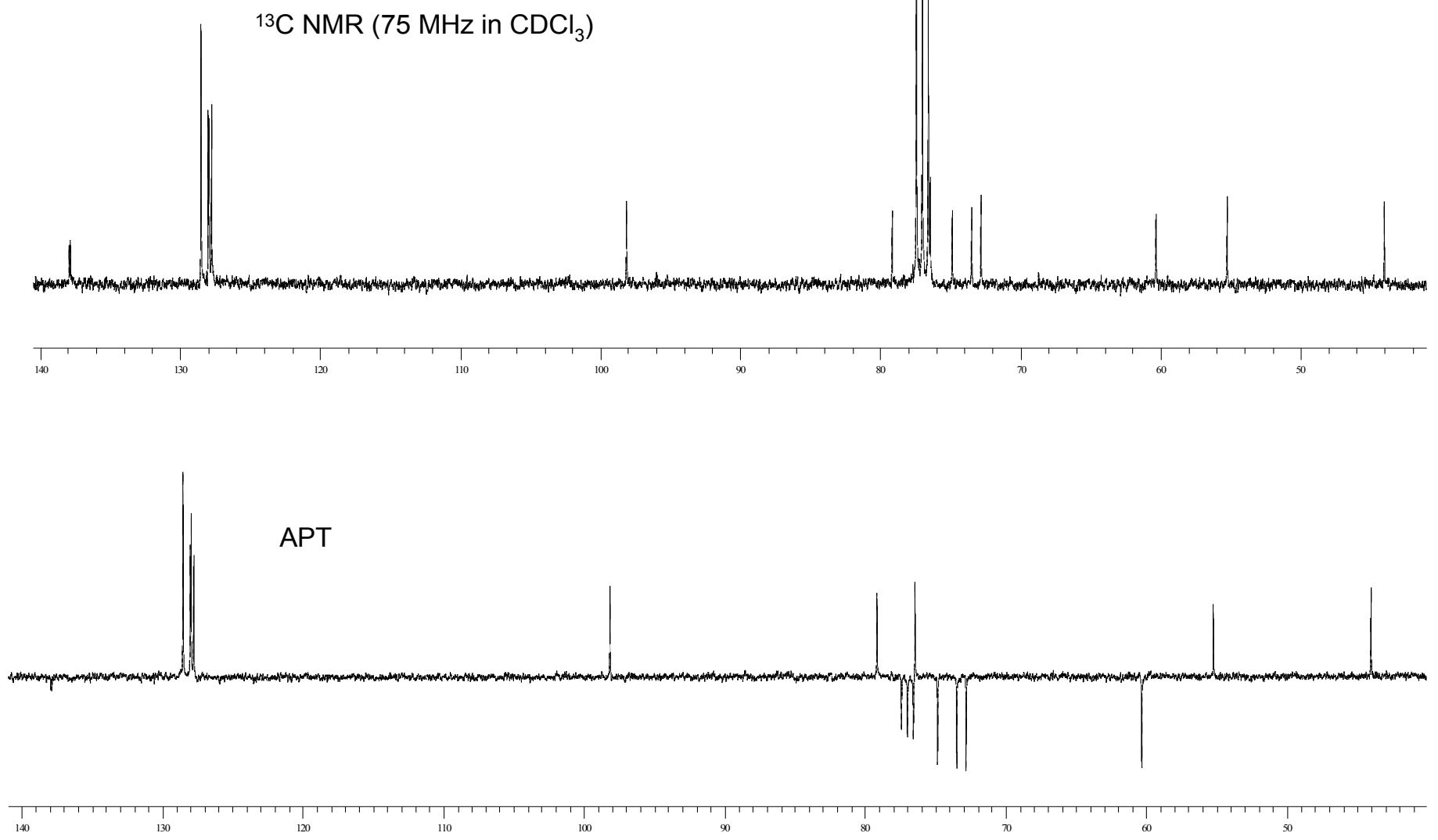


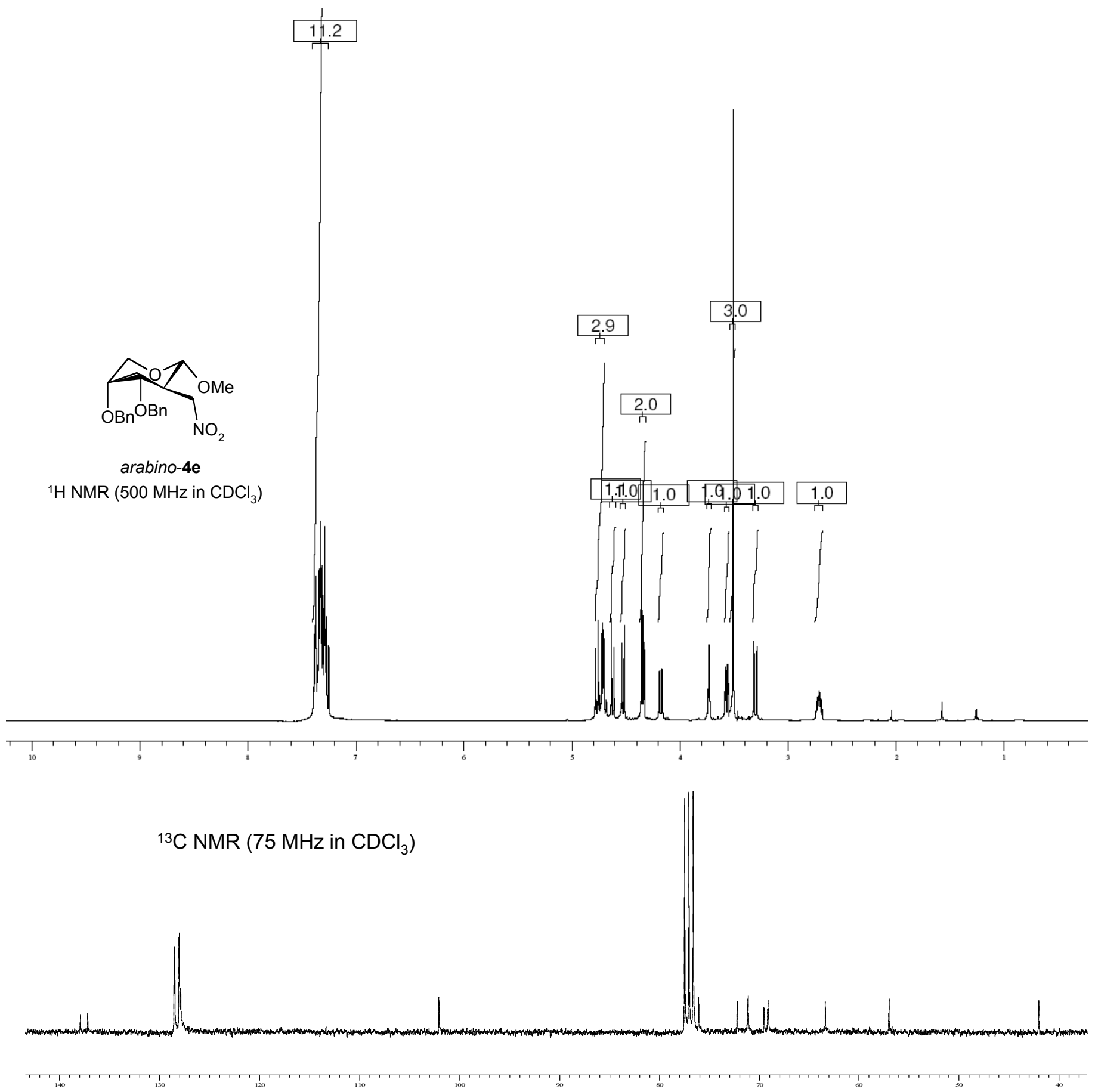

APT
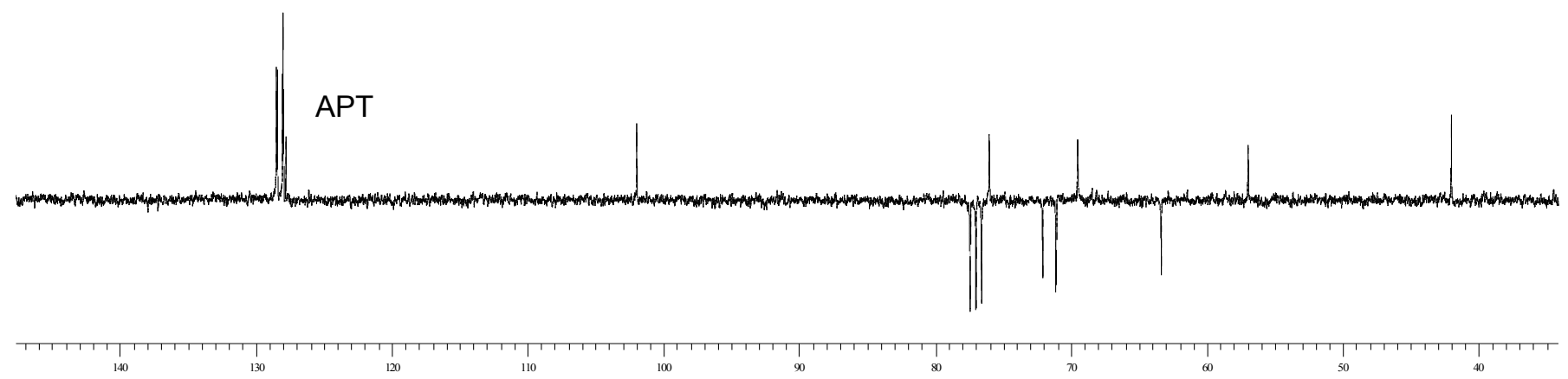

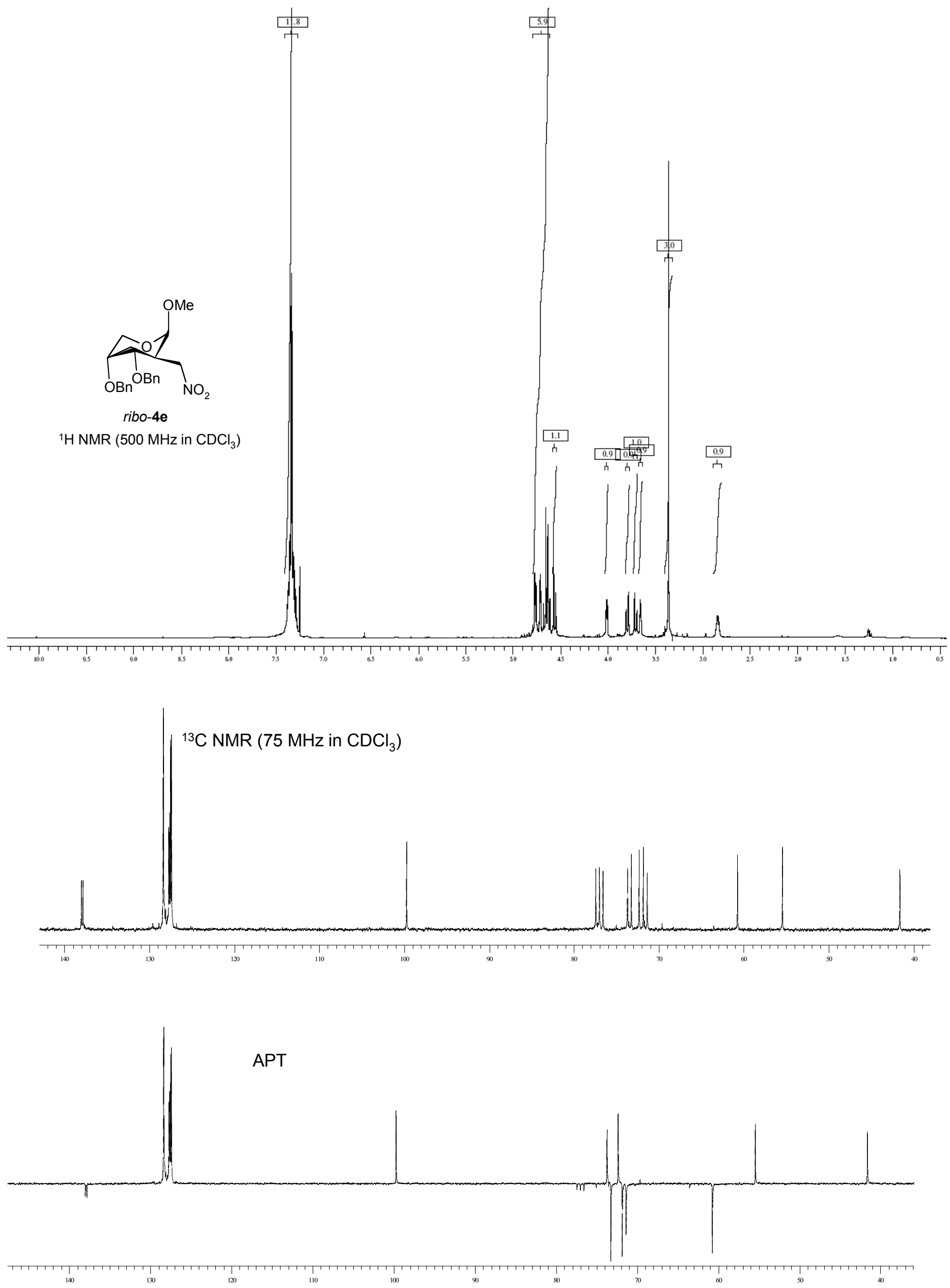

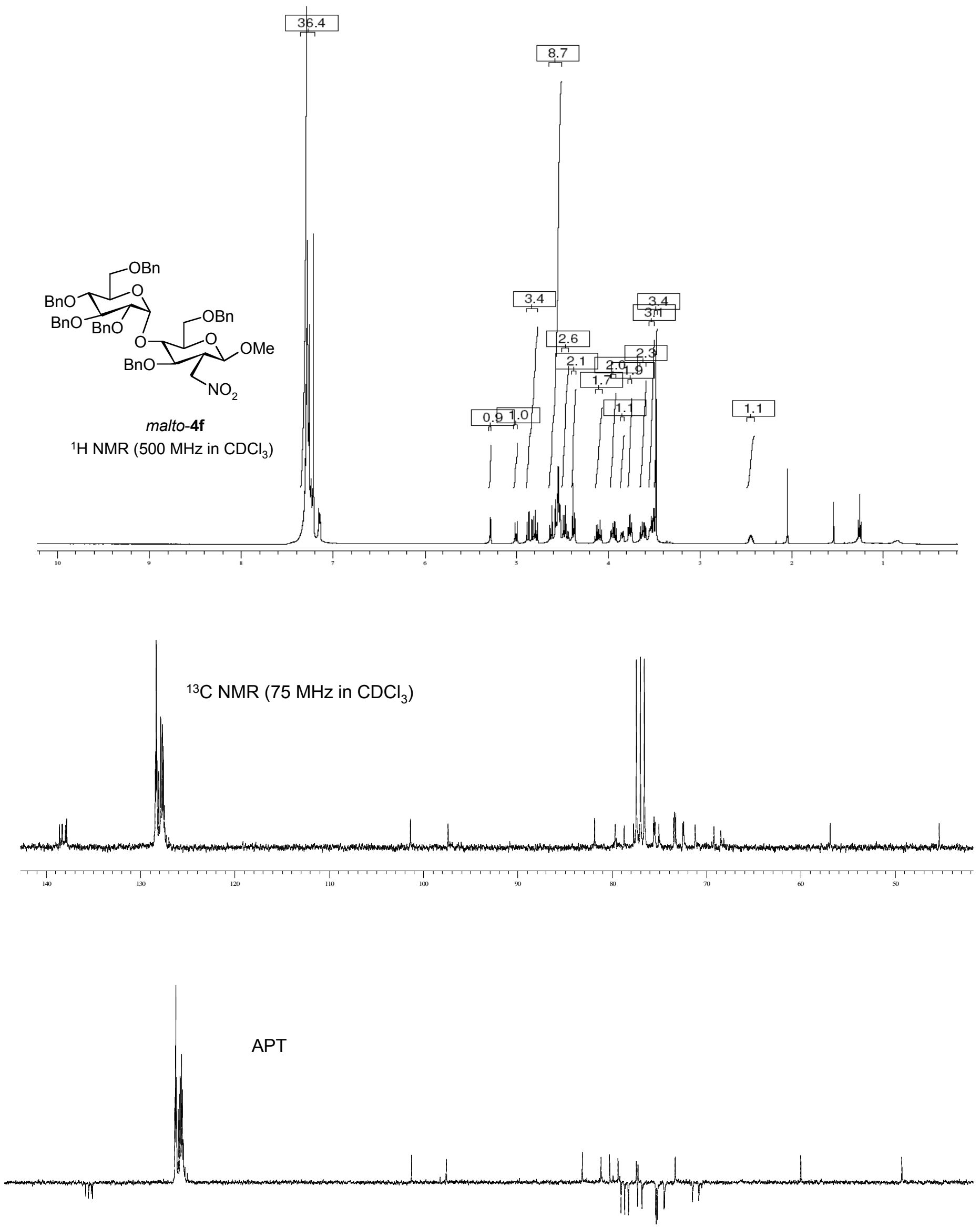

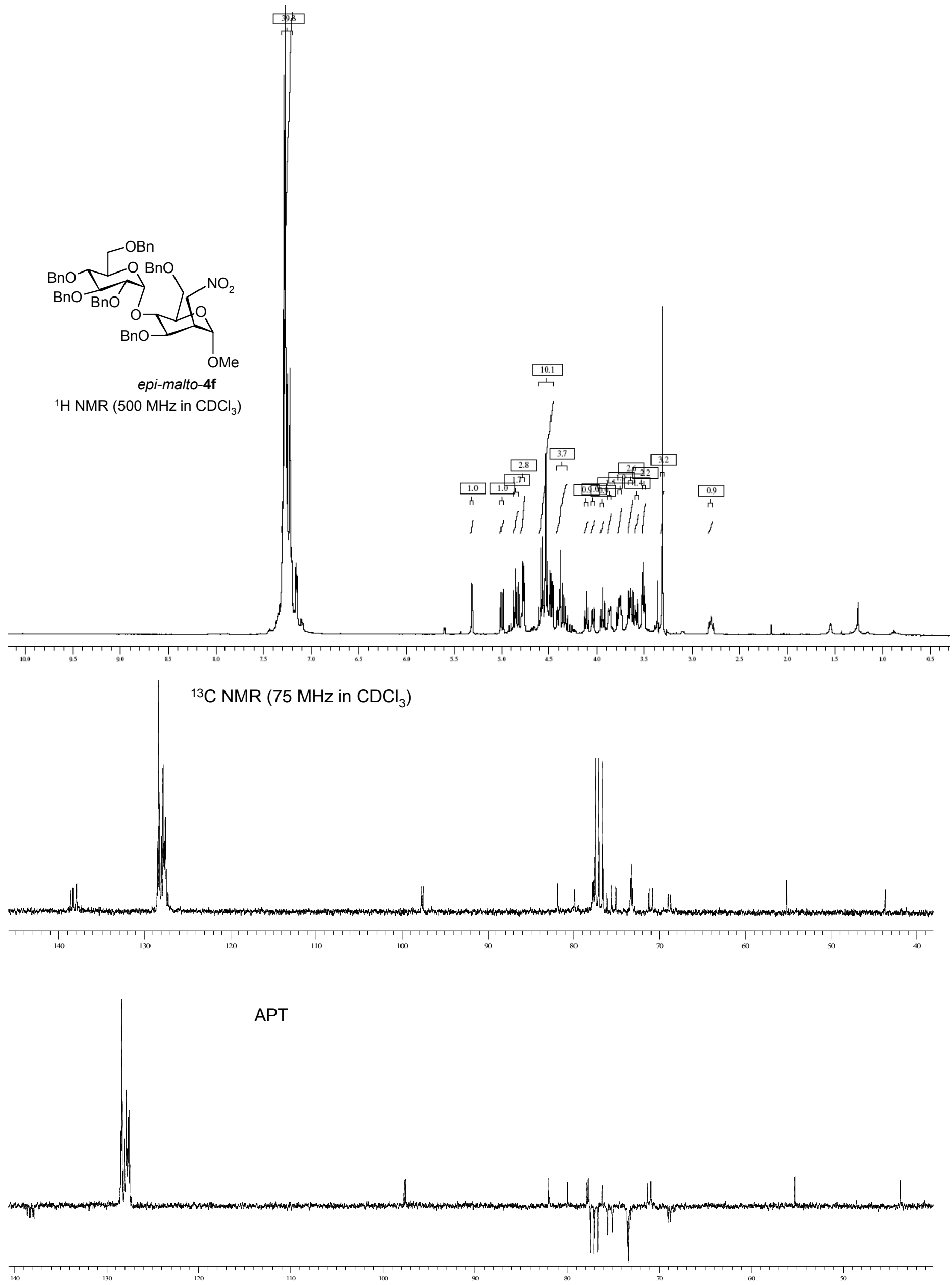

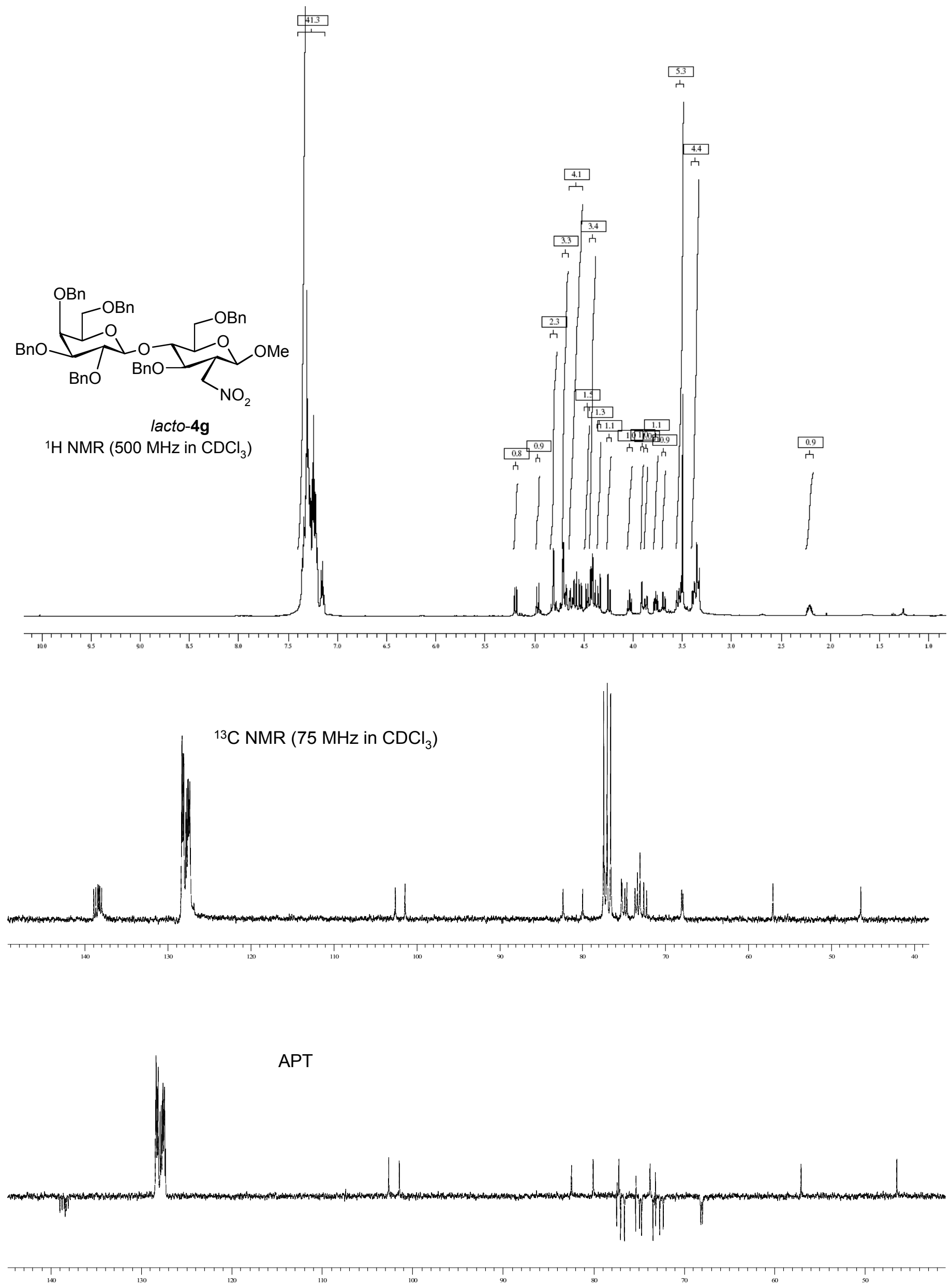

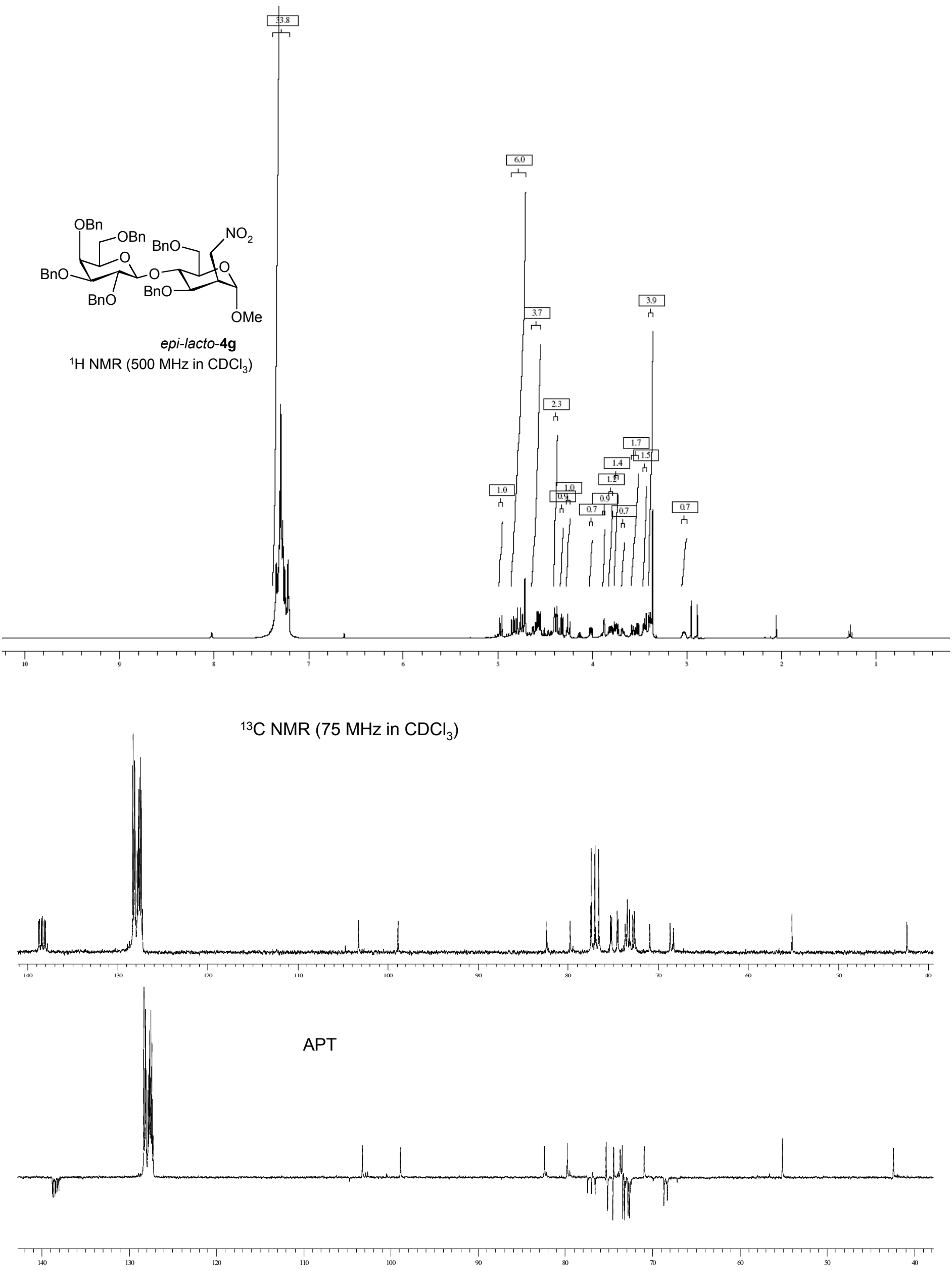

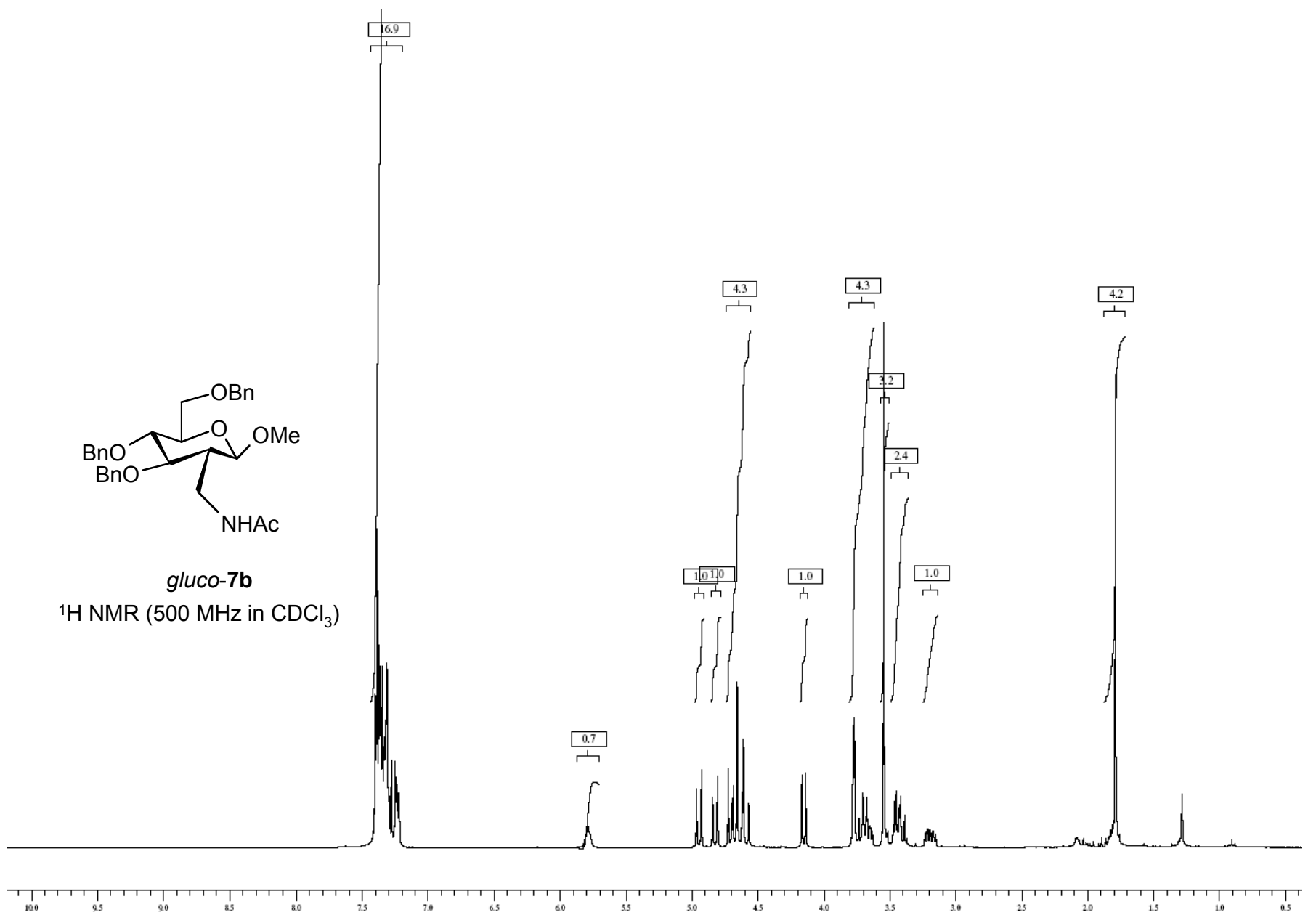

${ }^{13} \mathrm{C}$ NMR $\left(75 \mathrm{MHz}\right.$ in $\mathrm{CDCl}_{3}$ )
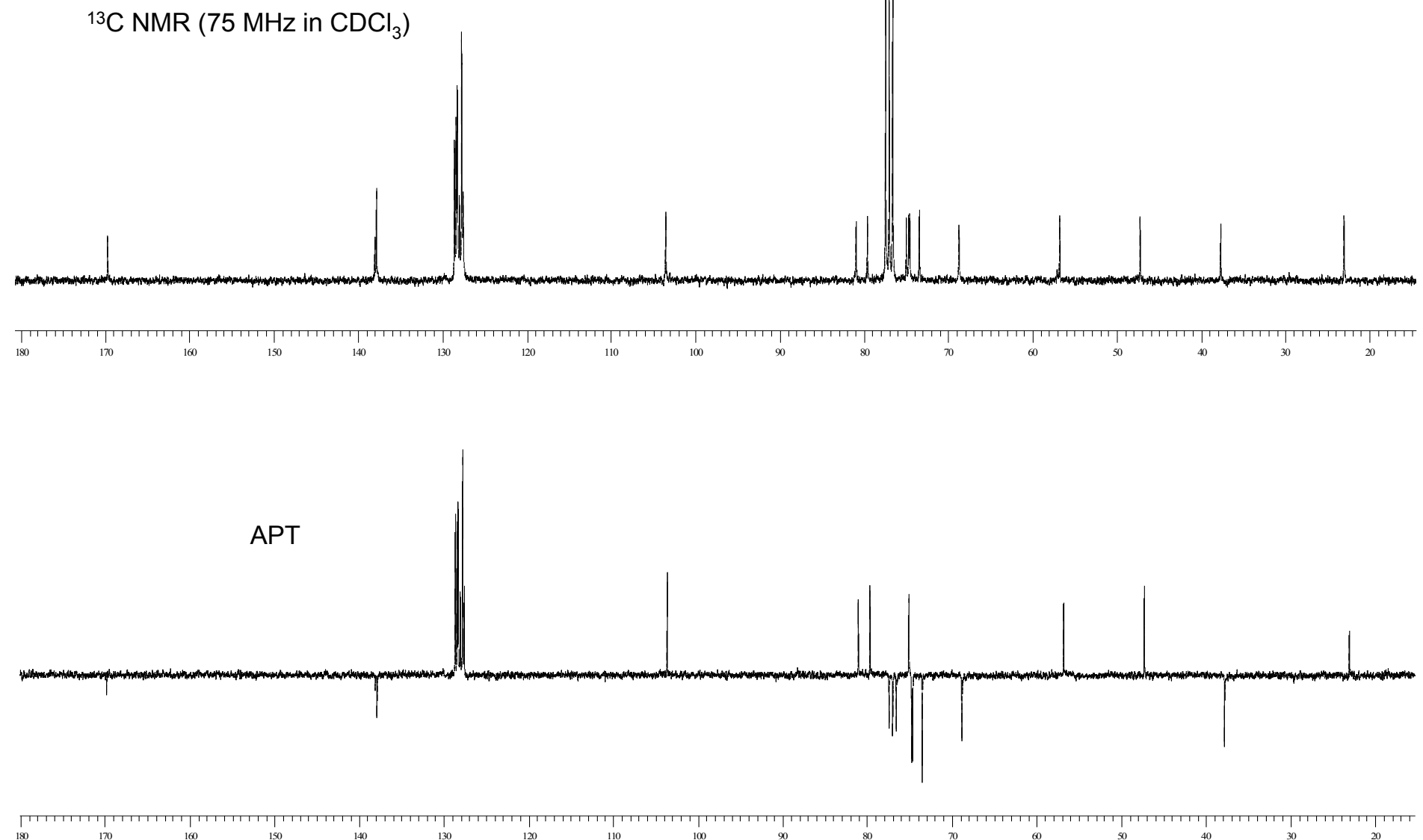


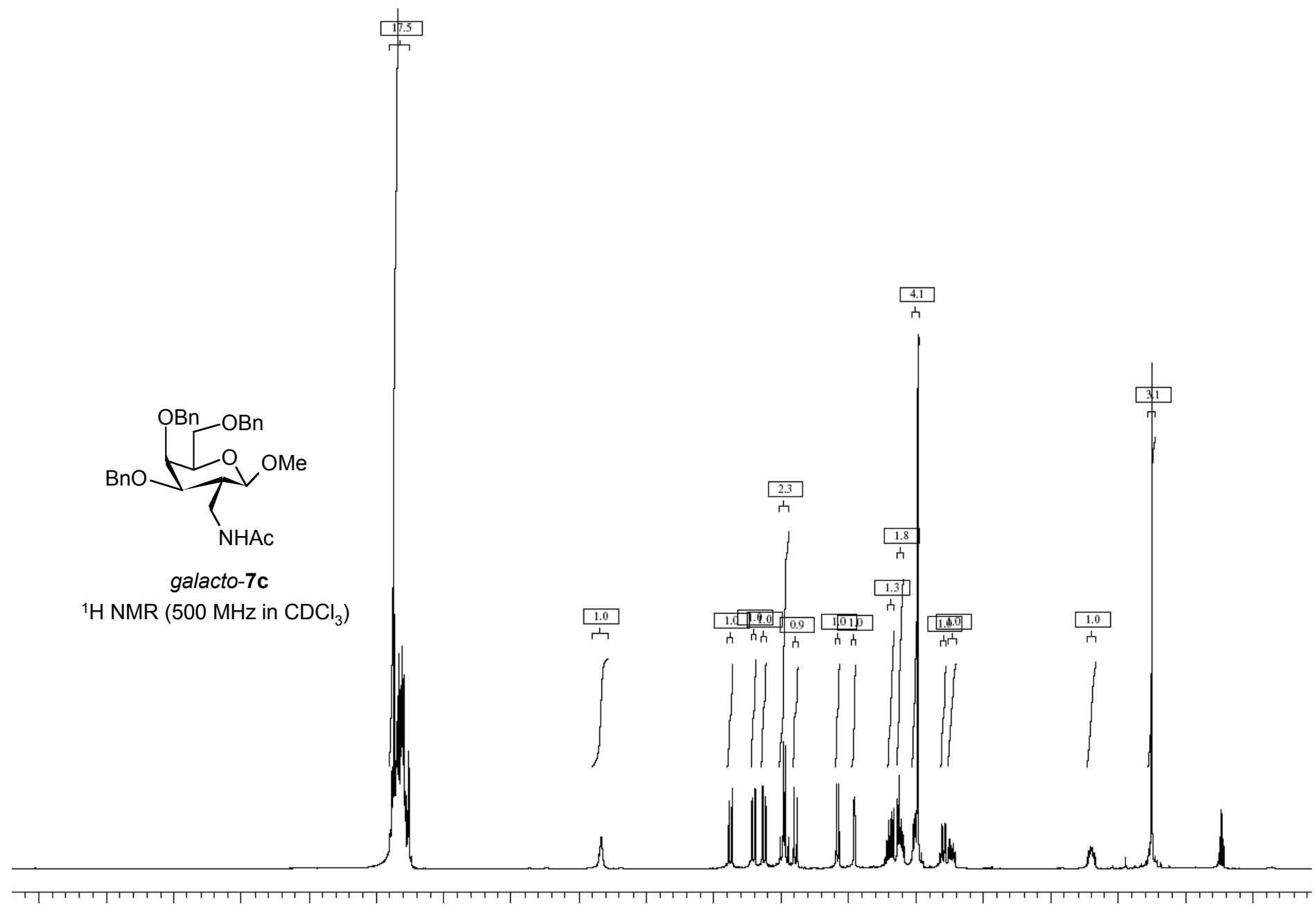

${ }^{13} \mathrm{C} \mathrm{NMR} \mathrm{(75} \mathrm{MHz}$ in $\mathrm{CDCl}_{3}$ )
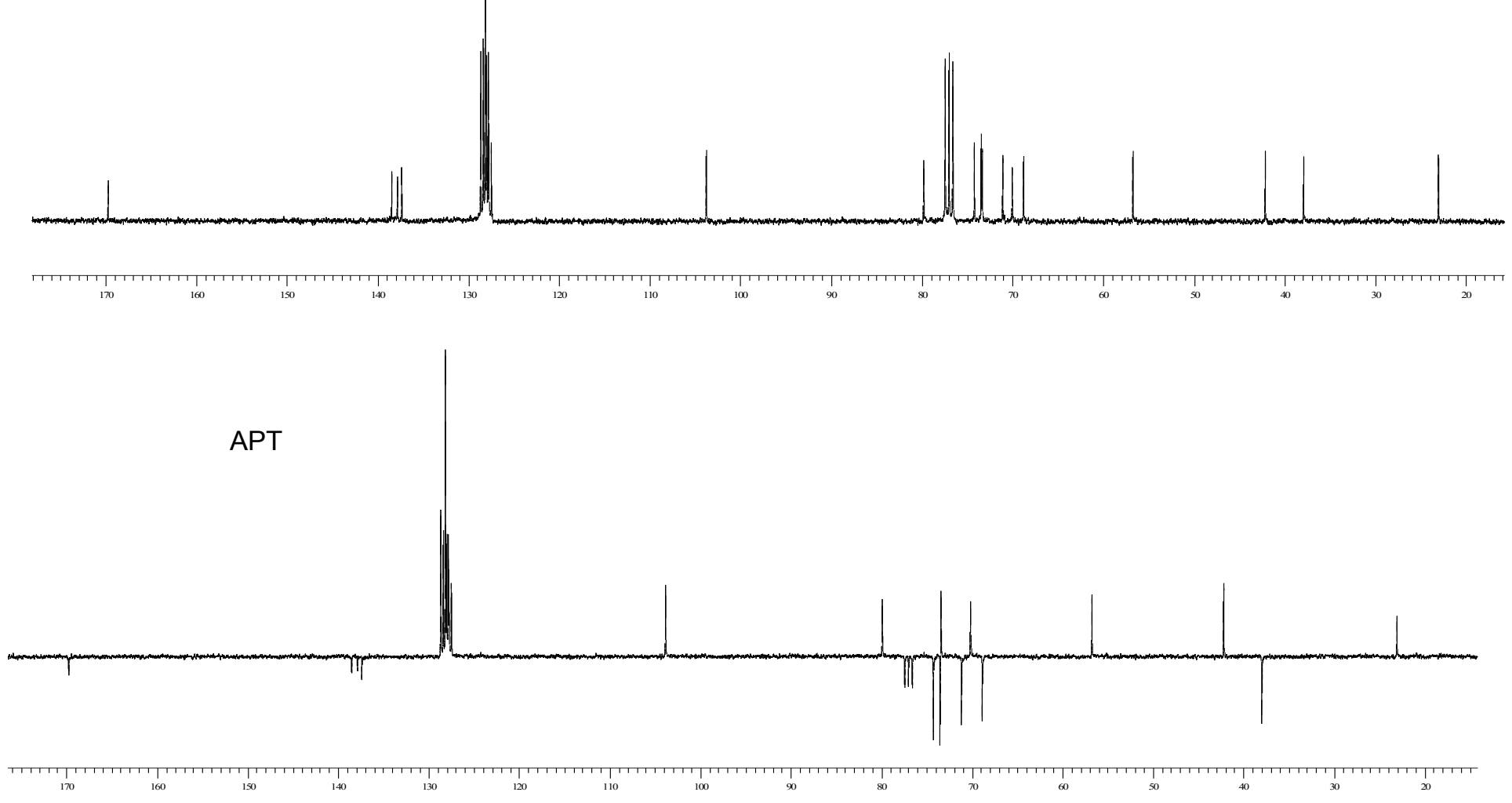


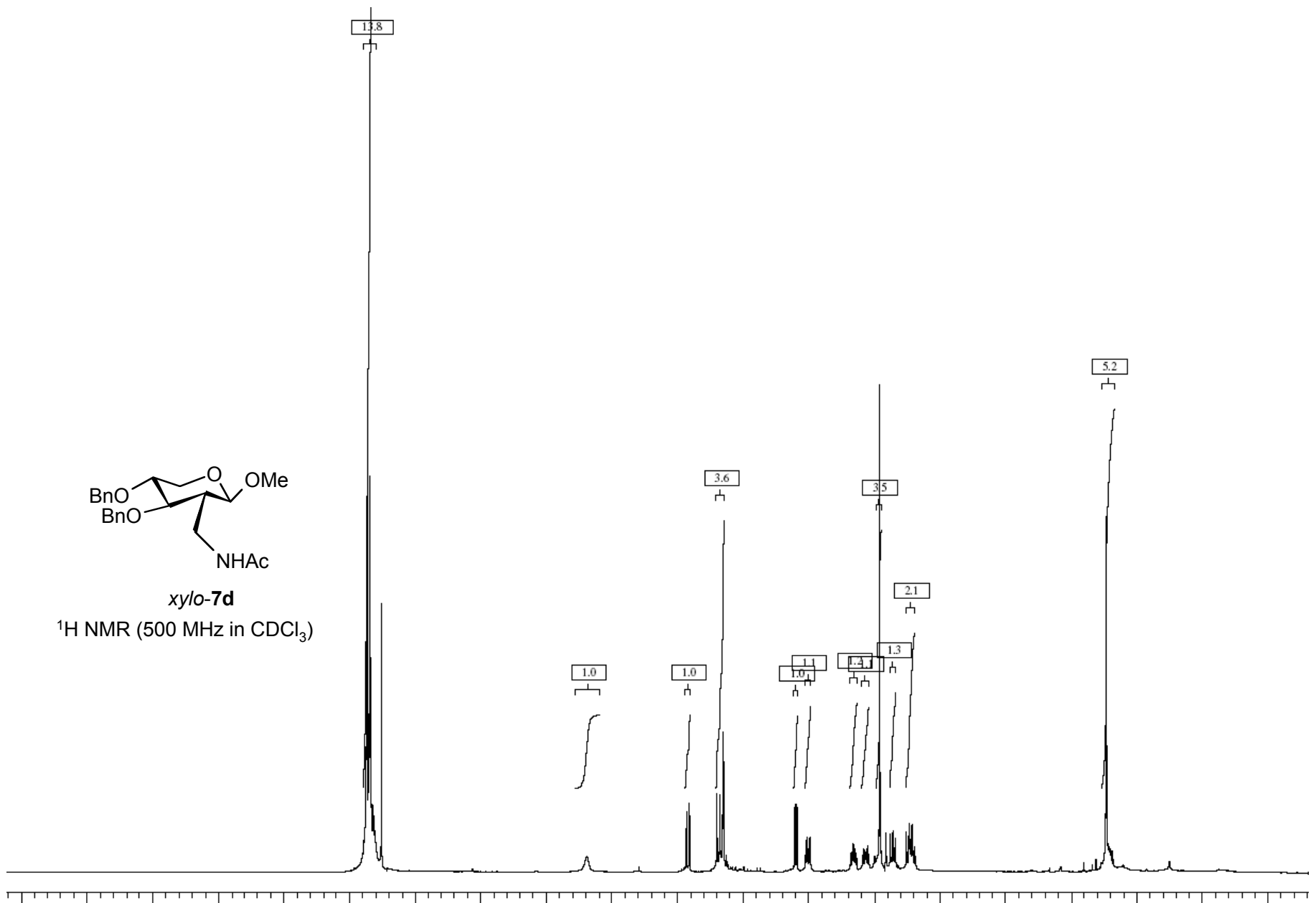

${ }^{13} \mathrm{C}$ NMR $\left(75 \mathrm{MHz}\right.$ in $\mathrm{CDCl}_{3}$ )

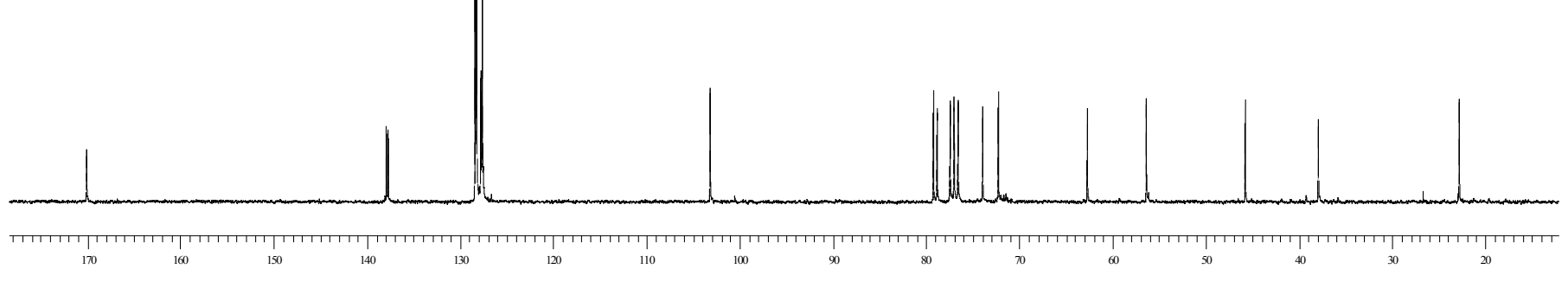

APT

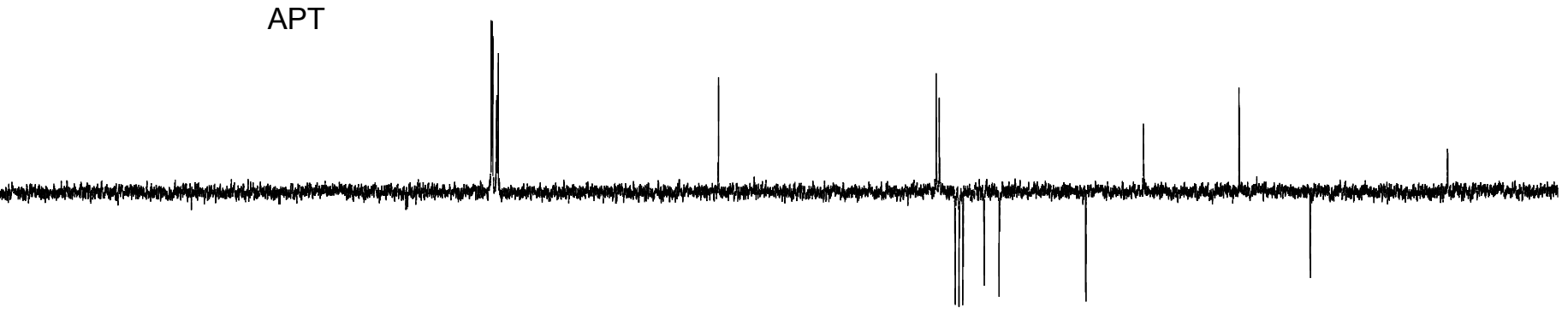




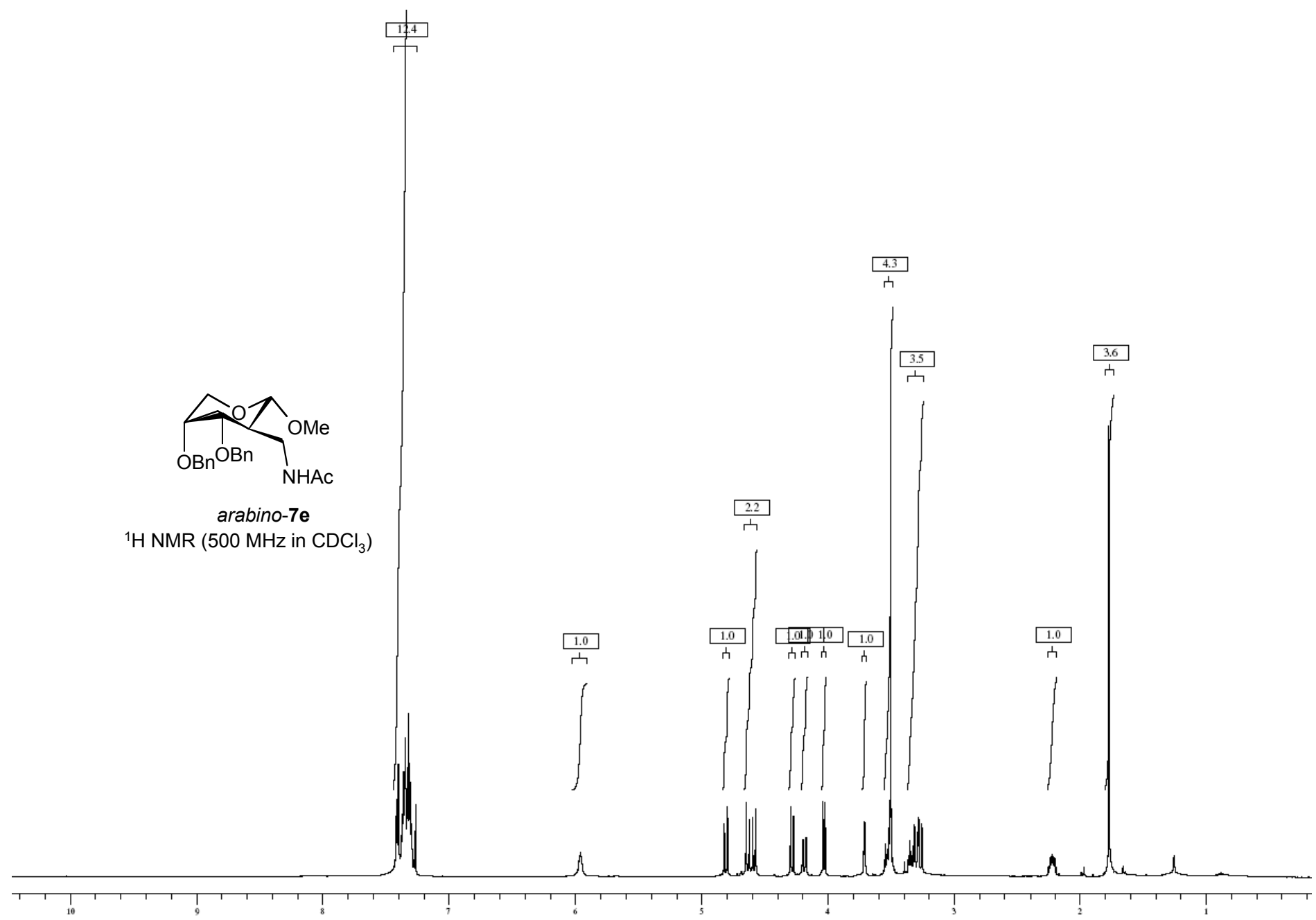

${ }^{13} \mathrm{C}$ NMR $\left(75 \mathrm{MHz}\right.$ in $\mathrm{CDCl}_{3}$ )
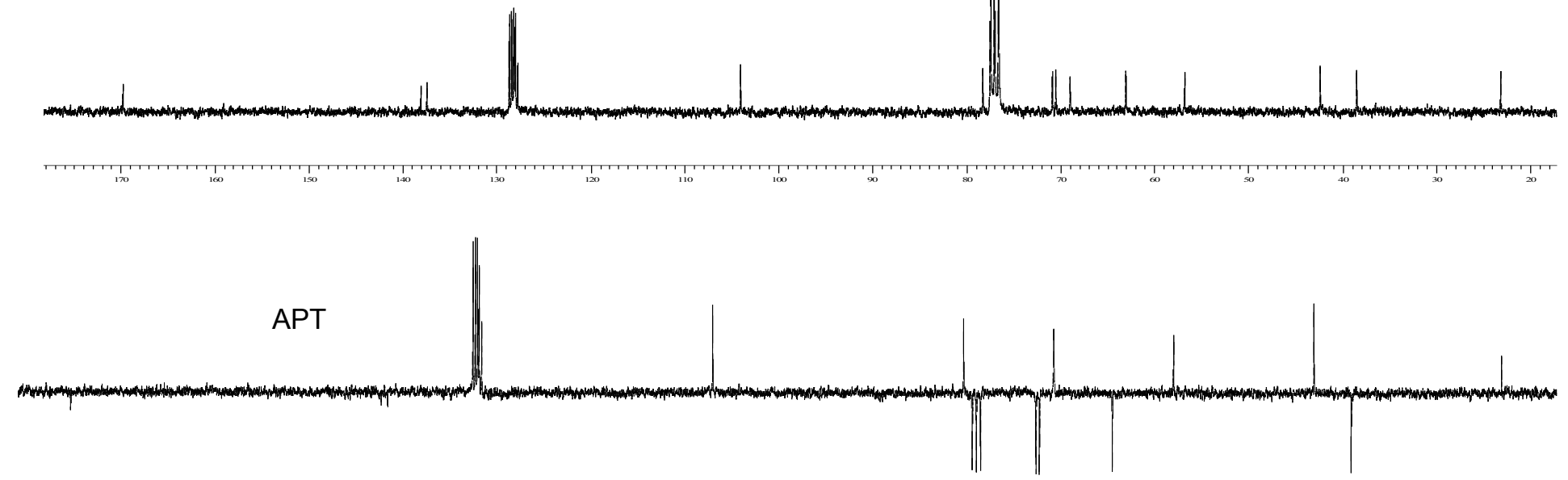

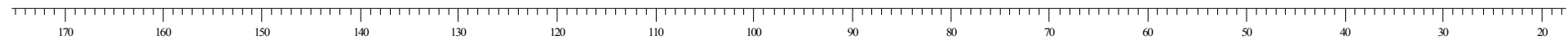



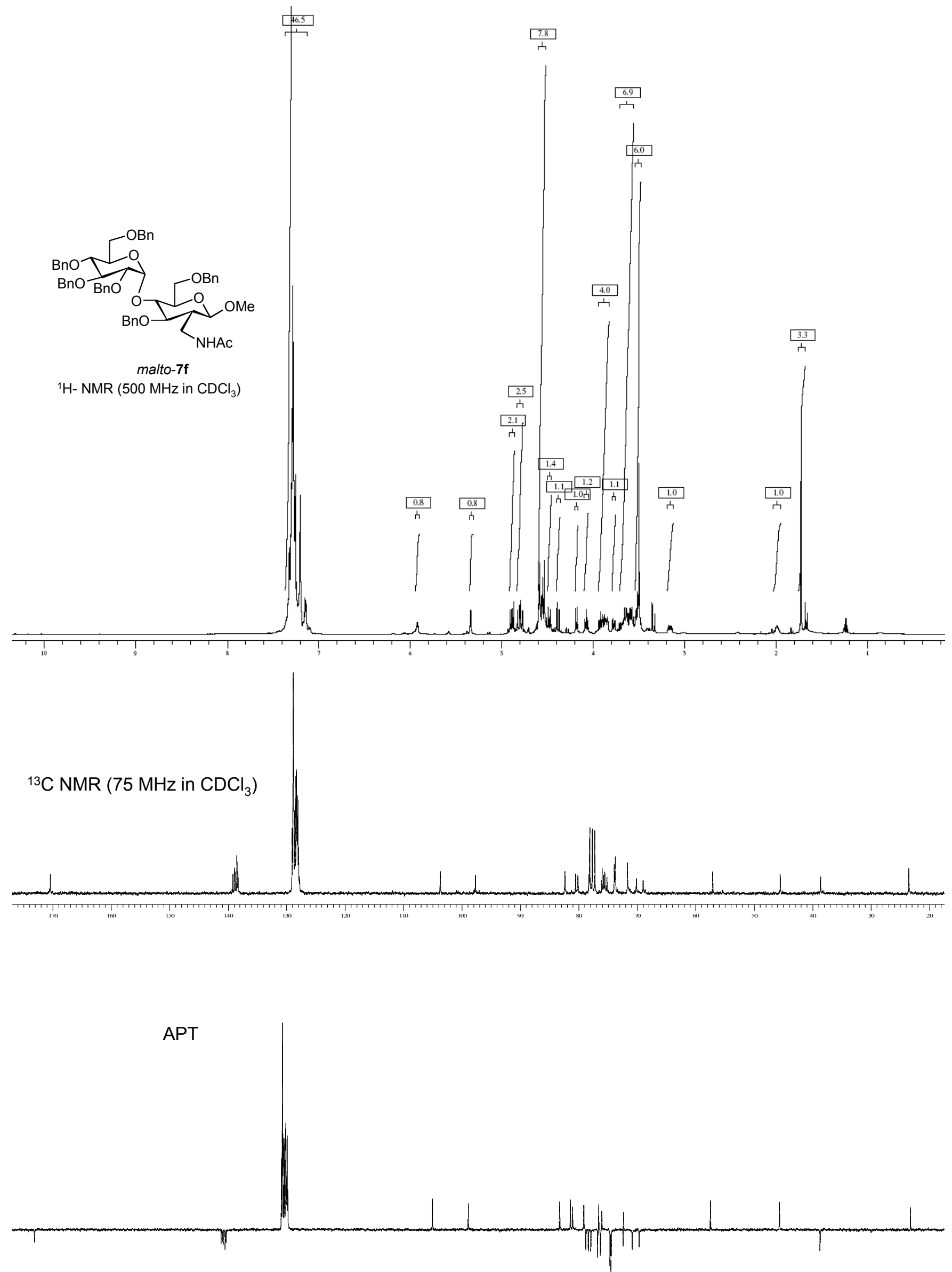

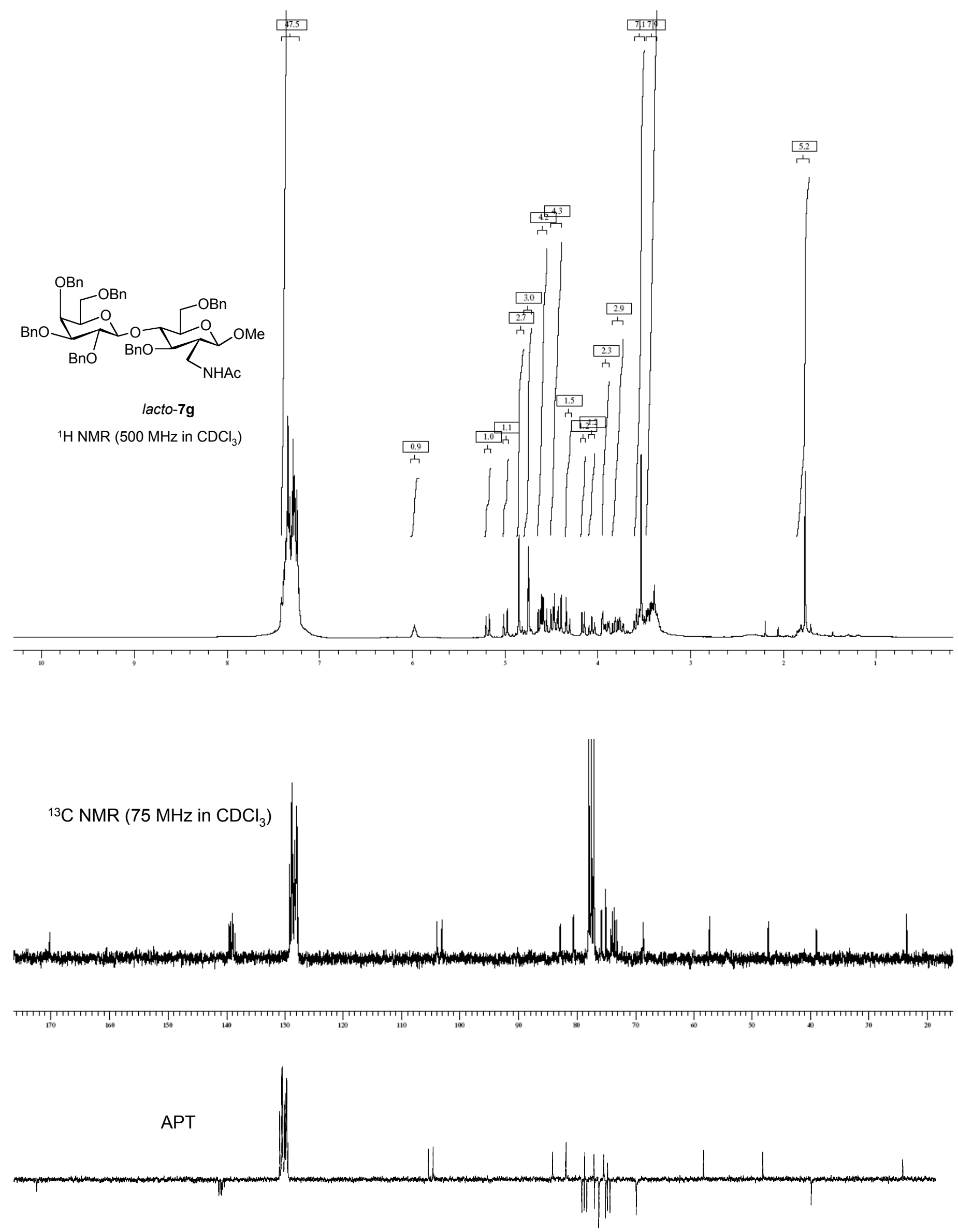


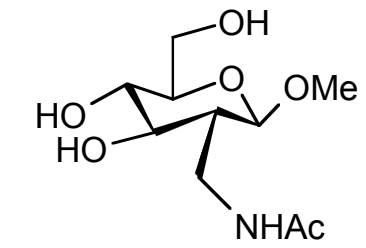

gluco-1b

${ }^{1} \mathrm{H}$ NMR $\left(500 \mathrm{MHz}\right.$ in $\left.\mathrm{D}_{2} \mathrm{O}\right)$

${ }^{13} \mathrm{C}$ NMR $\left(75 \mathrm{MHz}\right.$ in $\left.\mathrm{D}_{2} \mathrm{O}\right)$
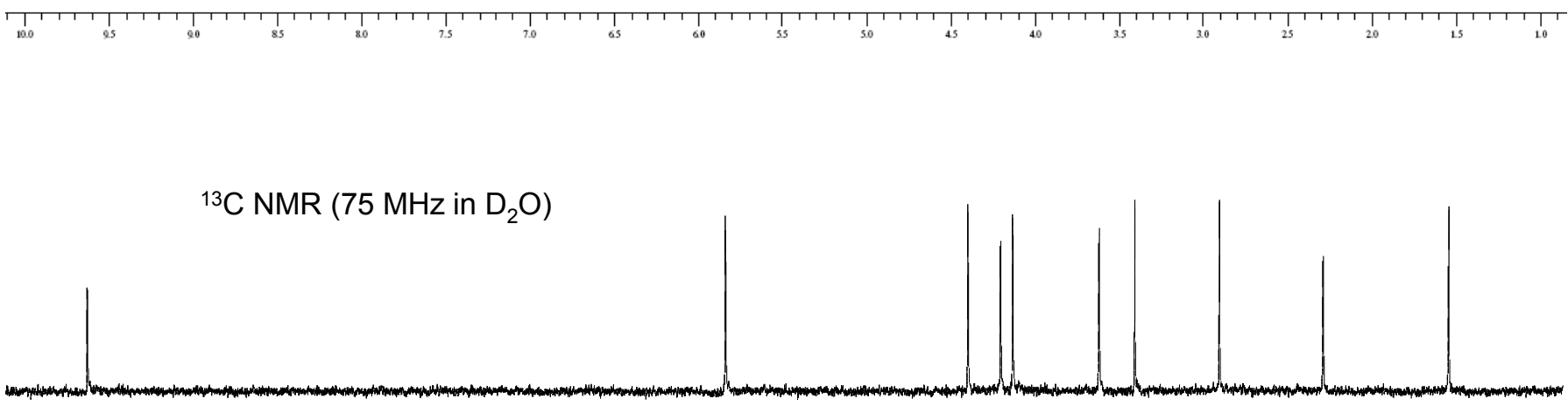

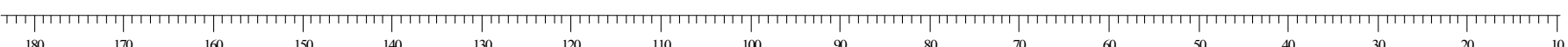
APT

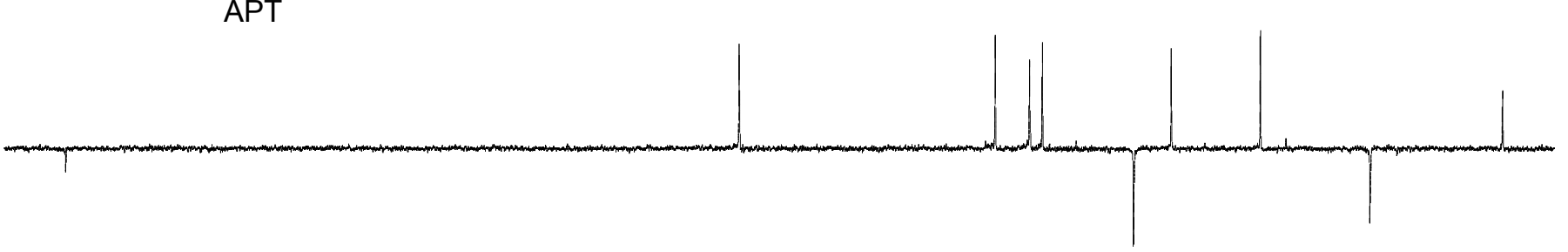

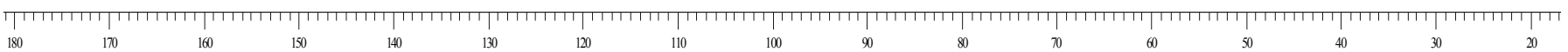




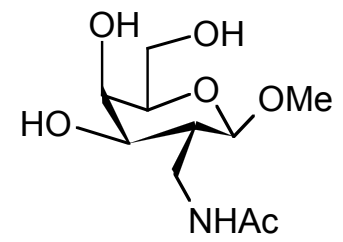

galacto-1c

${ }^{1} \mathrm{H}$ NMR $\left(500 \mathrm{MHz}\right.$ in $\left.\mathrm{D}_{2} \mathrm{O}\right)$

${ }^{13} \mathrm{C}$ NMR $\left(75 \mathrm{MHz}\right.$ in $\left.\mathrm{D}_{2} \mathrm{O}\right)$
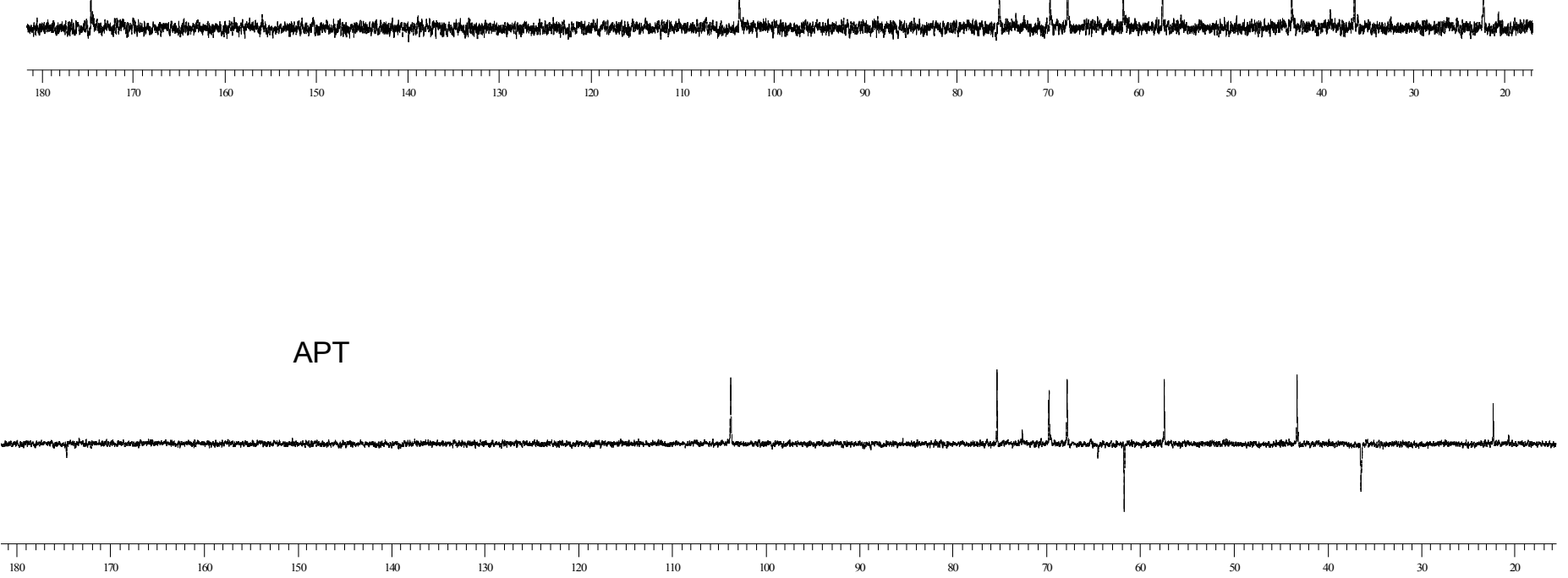


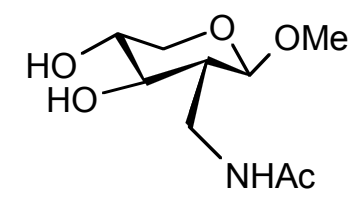

xylo-1d

${ }^{1} \mathrm{H}$ NMR $\left(500 \mathrm{MHz}\right.$ in $\left.\mathrm{D}_{2} \mathrm{O}\right)$

${ }^{13} \mathrm{C} \operatorname{NMR}\left(75 \mathrm{MHz}\right.$ in $\left.\mathrm{D}_{2} \mathrm{O}\right)$

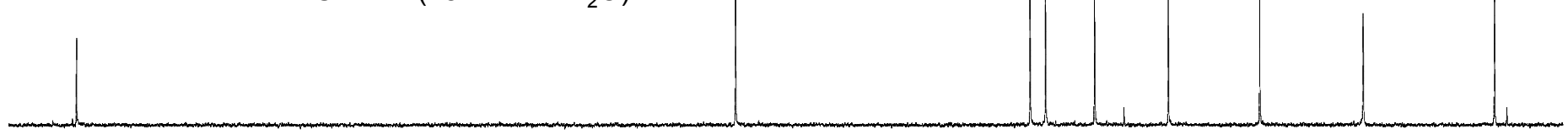

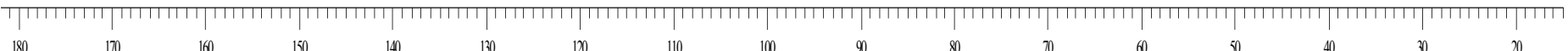

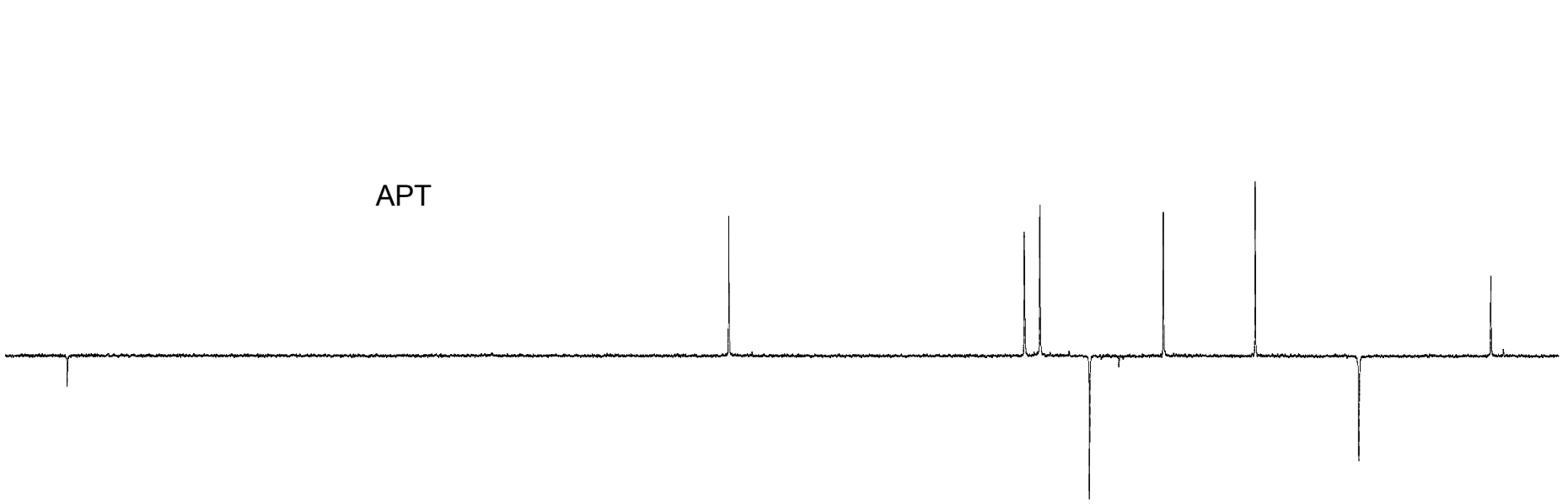




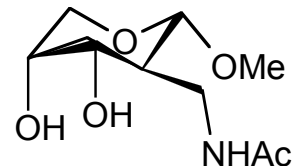

arabino-1e

${ }^{1} \mathrm{H}$ NMR $\left(500 \mathrm{MHz}\right.$ in $\left.\mathrm{D}_{2} \mathrm{O}\right)$
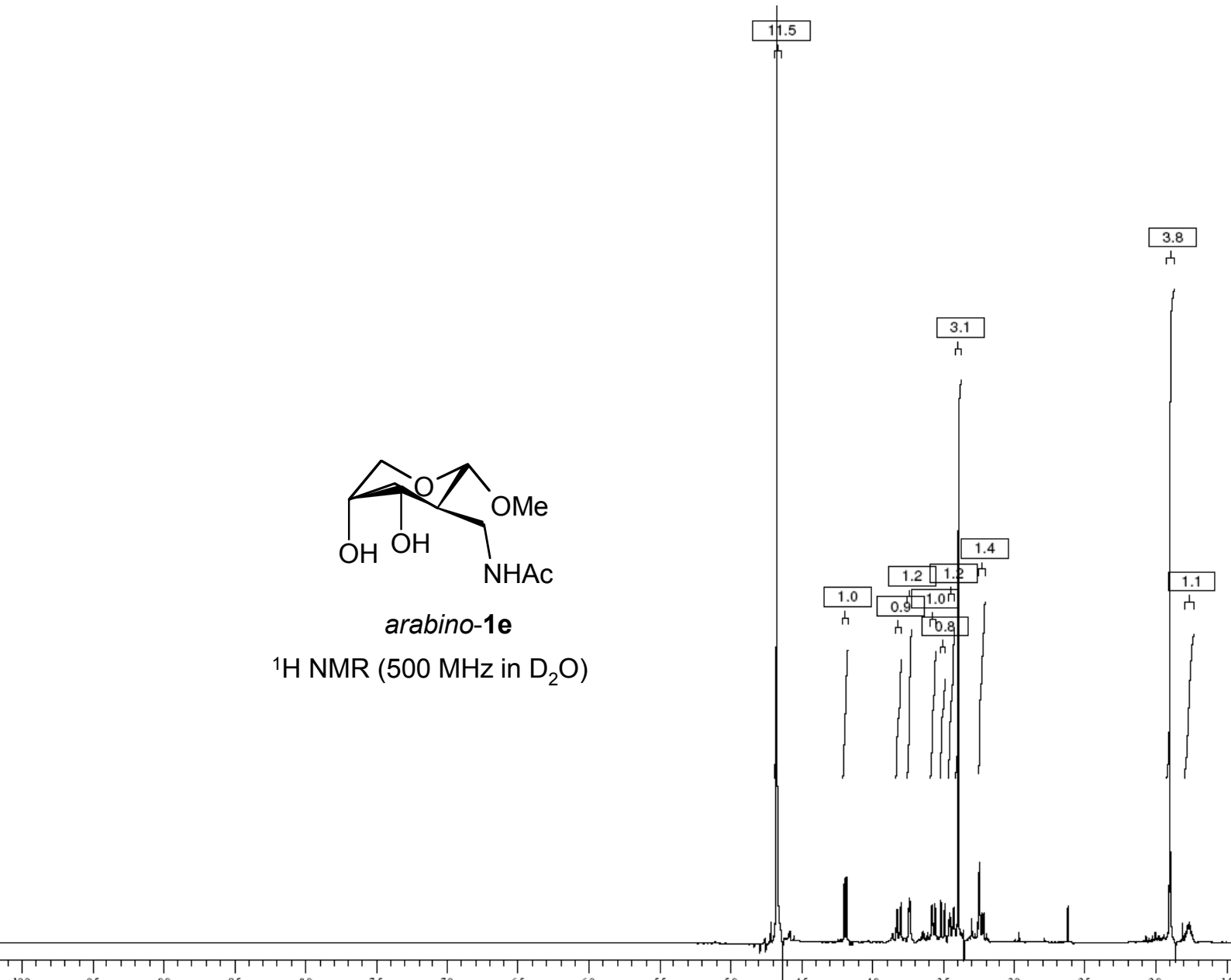

${ }^{13} \mathrm{C}$ NMR $\left(75 \mathrm{MHz}\right.$ in $\left.\mathrm{D}_{2} \mathrm{O}\right)$

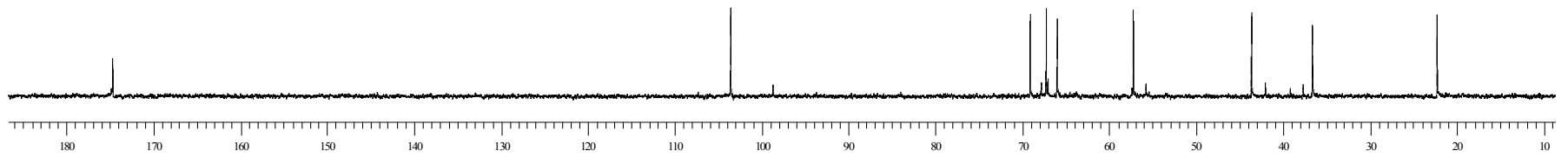

APT

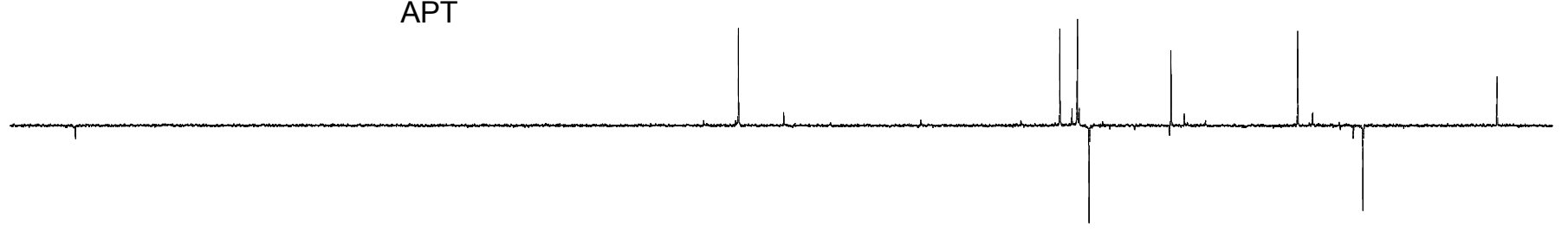




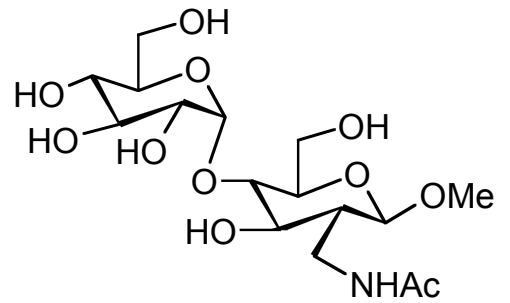

malto-1f

${ }^{1} \mathrm{H}$ NMR $\left(500 \mathrm{MHz}\right.$ in $\left.\mathrm{D}_{2} \mathrm{O}\right)$

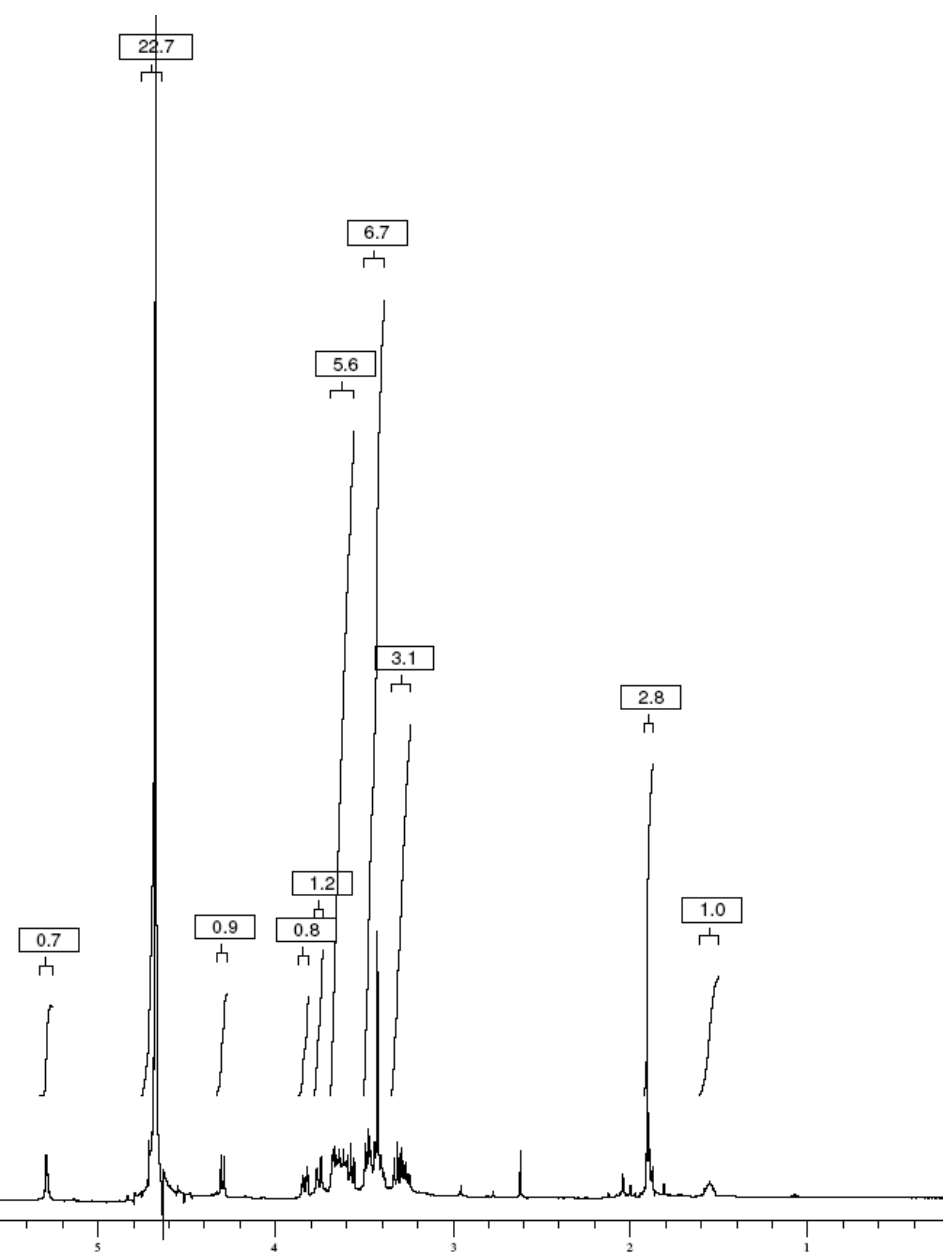

${ }^{13} \mathrm{C}$ NMR $\left(75 \mathrm{MHz}\right.$ in $\left.\mathrm{D}_{2} \mathrm{O}\right)$

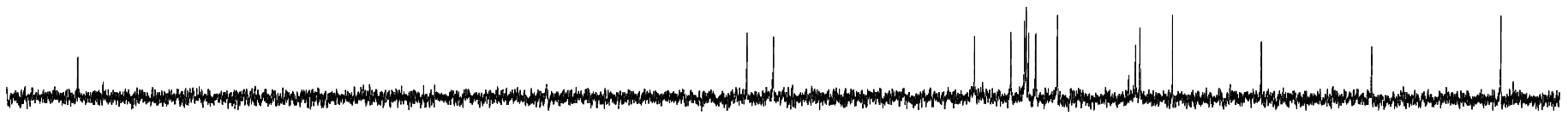

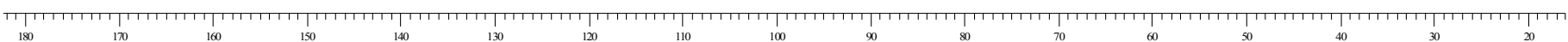
APT
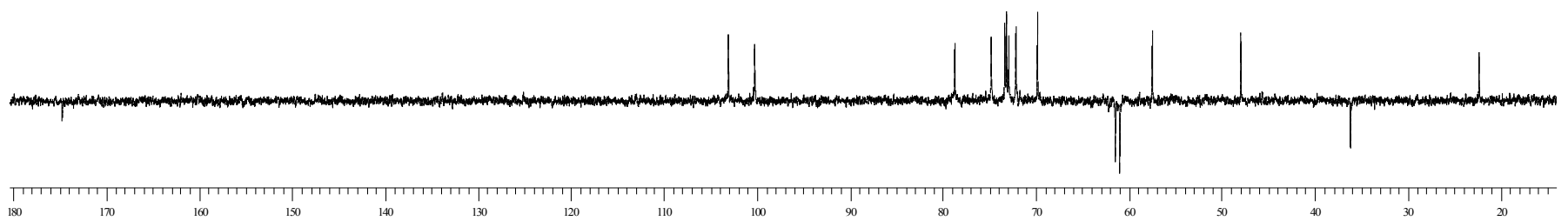


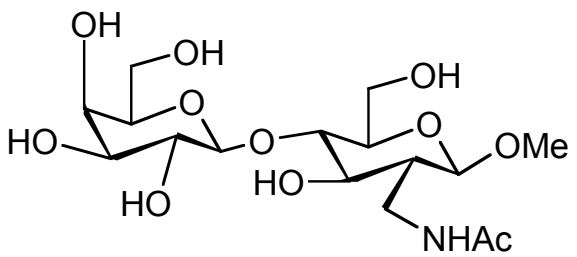

lacto-1g

${ }^{1} \mathrm{H}$ NMR $\left(500 \mathrm{MHz}\right.$ in $\left.\mathrm{D}_{2} \mathrm{O}\right)$

${ }^{13} \mathrm{C}$ NMR $\left(75 \mathrm{MHz}\right.$ in $\left.\mathrm{D}_{2} \mathrm{O}\right)$
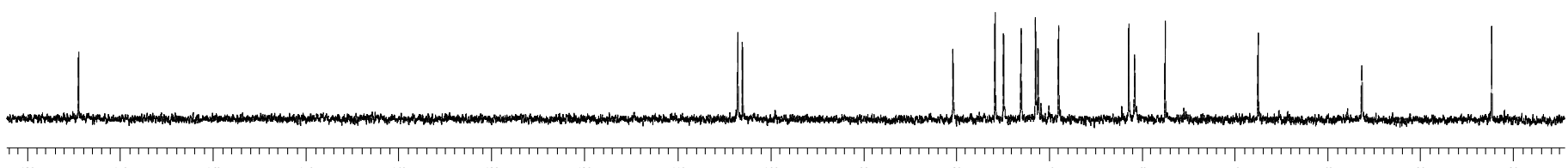

$180-170-100$
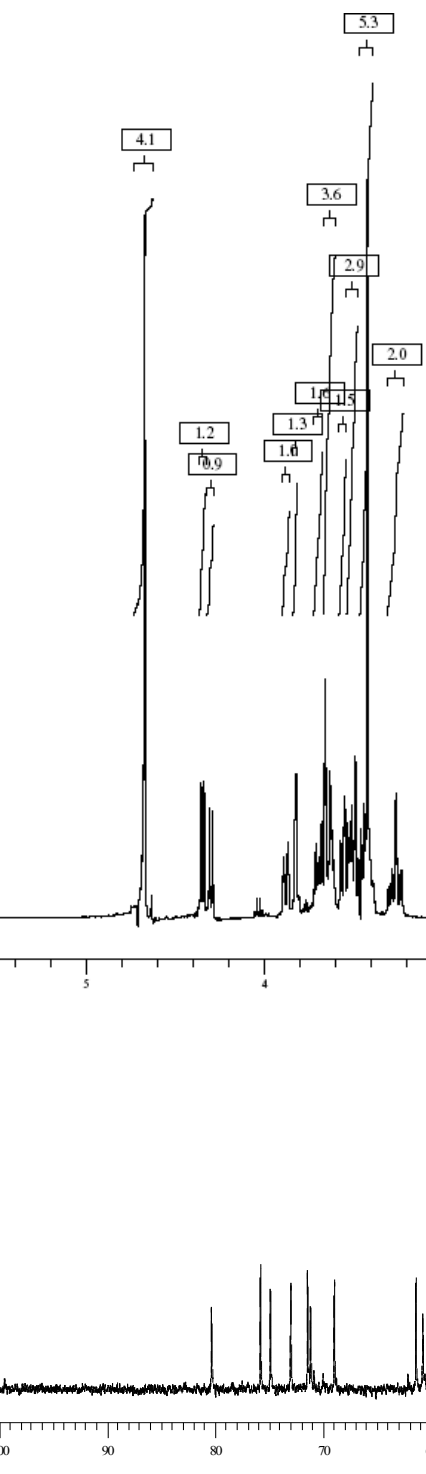

Lhe

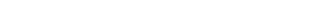

APT
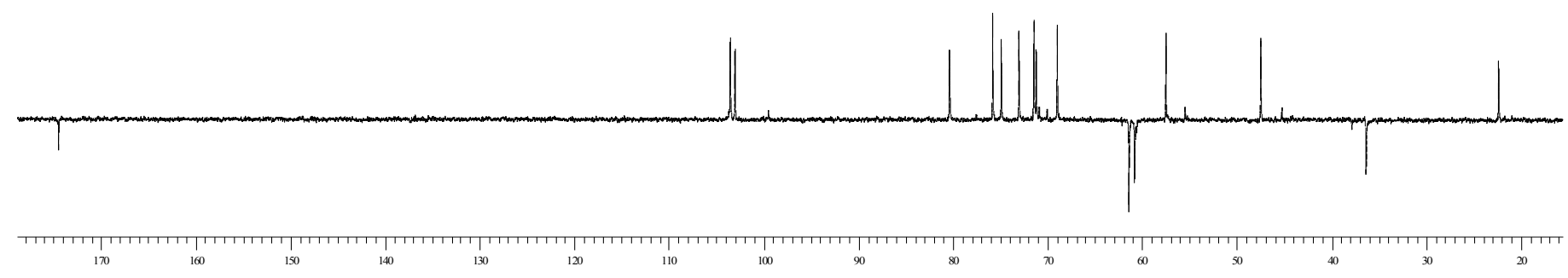